San Jose State University

SJSU ScholarWorks

Master's Theses

Master's Theses and Graduate Research

Summer 2013

\title{
Effects of Compression on the Temperature Distribution of a Tissue-Mimicking Material During High-Intensity Focused Ultrasound (HIFU) Ablation
}

Austin Robbins Roth

San Jose State University

Follow this and additional works at: https://scholarworks.sjsu.edu/etd_theses

\section{Recommended Citation}

Roth, Austin Robbins, "Effects of Compression on the Temperature Distribution of a Tissue-Mimicking Material During High-Intensity Focused Ultrasound (HIFU) Ablation" (2013). Master's Theses. 4362. DOI: https://doi.org/10.31979/etd.c8d3-jfdu https://scholarworks.sjsu.edu/etd_theses/4362

This Thesis is brought to you for free and open access by the Master's Theses and Graduate Research at SJSU ScholarWorks. It has been accepted for inclusion in Master's Theses by an authorized administrator of SJSU ScholarWorks. For more information, please contact scholarworks@sjsu.edu. 


\title{
EFFECTS OF COMPRESSION ON THE TEMPERATURE DISTRIBUTION OF A TISSUE-MIMICKING MATERIAL DURING HIGH-INTENSITY FOCUSED ULTRASOUND (HIFU) ABLATION
}

\begin{abstract}
A Thesis
Presented to

The Faculty of the Department of Biomedical, Chemical \& Materials Engineering San Jose State University
\end{abstract}

\author{
In partial fulfillment \\ of the requirements for the degree \\ Master of Science
}

by

Austin R. Roth

August 2013 
(C) 2013

Austin R. Roth

ALL RIGHTS RESERVED 
The Designated Committee Approves the Thesis Titled

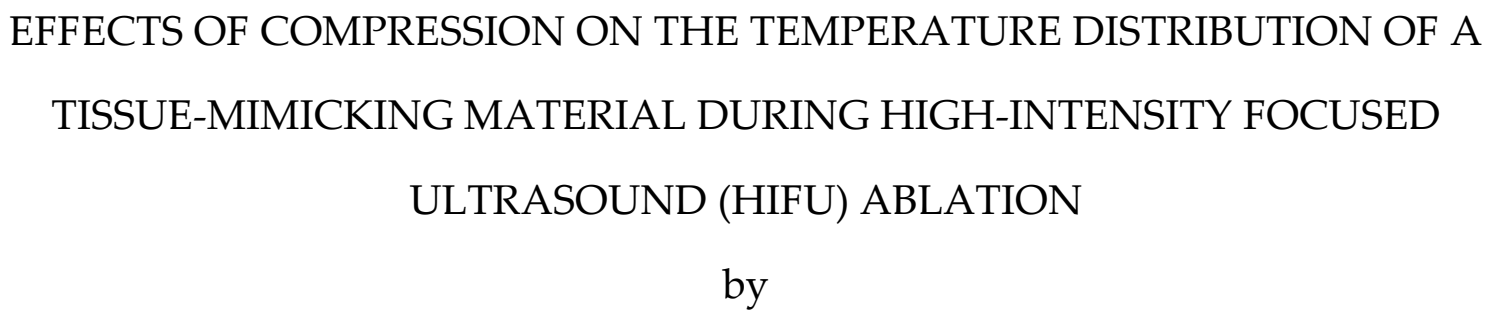

APPROVED FOR THE DEPARTMENT OF BIOMEDICAL, CHEMICAL \& MATERIALS ENGINEERING SAN JOSÉ STATE UNIVERSITY

August 2013

$\begin{array}{ll}\text { Dr. Sang-Joon John Lee } & \text { Department of Mechanical Engineering } \\ \text { Dr. Mallika Keralapura } & \text { Department of Electrical Engineering } \\ \text { Kevin Taylor } & \text { VP Development \& Operations, ReCor Medical }\end{array}$ 


\section{ABSTRACT \\ EFFECTS OF COMPRESSION ON THE TEMPERATURE DISTRUBUTION OF A PERFUSED TISSUE-MIMICKING MATERIAL DURING HIGH-INTENSITY FOCUSED ULTRASOUND (HIFU) ABLATION}

Local blood flow near a high-intensity focused ultrasound (HIFU) target has been shown to decrease ablation effectiveness and predictability, creating a barrier to clinical use for breast cancer treatment. This study investigated the effects of compression on HIFU ablation of a perfused tissue-mimicking material. Gellan gum-based phantoms, with thermal and acoustic properties similar to those of soft tissue, were ablated with a $1.13 \mathrm{MHz}$ HIFU transducer while being subjected to varying levels of external compression. Phantoms were designed with an embedded $6 \mathrm{~mm}$ diameter vessel meant to mimic a thermally significant blood vessel near a breast tumor. The internal temperature profile was measured using T-type thin-wire thermocouples embedded in the phantom along the transverse axis. The temperature distributions on opposing lateral sides of the HIFU focal point were measured to determine the effects of compression on heating symmetry. After heating with $30 \mathrm{~W}$ for $30 \mathrm{~s}$, the maximum discrepancy between a pair of thermocouples located $2 \mathrm{~mm}$ left and right of centerline, respectively, was $40^{\circ} \mathrm{C}$. This maximum discrepancy was observed at a fluid flow rate of $38 \mathrm{~mL} / \mathrm{min}$. With applied compression reducing flow to between 28 $\mathrm{mL} / \mathrm{min}$ and $25 \mathrm{~mL} / \mathrm{min}$, the discrepancy between left and right thermocouples was reduced to as low as $5.7^{\circ} \mathrm{C}$. Numerical predictions revealed an agreement with experimental results in the reduction of heating asymmetry as the flow rate decreased from $40 \mathrm{~mL} / \mathrm{min}$ to $20 \mathrm{~mL} / \mathrm{min}$. 


\section{ACKNOWLEDGEMENTS}

I would like to thank Dr. Mallika Keralapura and Dr. John Lee for their tireless effort in moving this thesis forward. Dr. Keralapura's support and weekly group meetings helped the project progress. Dr. Lee's critical mind and constructive criticisms were exactly what I needed while conducting this research and putting together the final thesis document. He also provided me, perhaps unknowingly, with many great examples of how to approach a problem and work through it.

I would also like to thank Kevin Taylor, my boss at ReCor Medical, for opening my eyes to the world of research and development. The experience I've gained at ReCor Medical, whether with using ultrasound devices, building test fixtures, or designing experimental procedures, has been a huge help in determining how to realistically set up my thesis experiments. It's also amazing to understand more clearly how technological advances and hard work can benefit patients who depend on the technology we study and develop. Thank you for that insight. 


\section{TABLE OF CONTENTS}

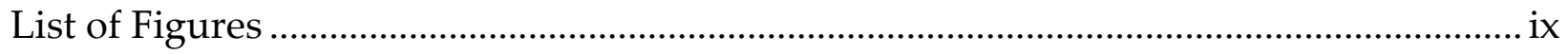

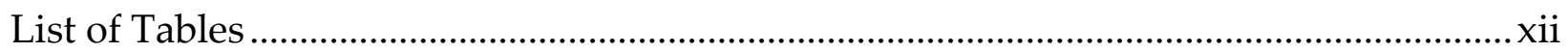

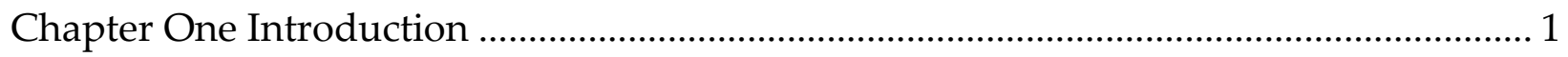

1.1 Introduction to Cancer Treatment and HIFU Therapy.............................................. 1

1.2 HIFU Physics and Transducer Design .................................................................. 3

1.2.1 Ultrasound Wave Propagation through Media...................................................... 5

1.2.2 Equations for Modeling Nonlinear Acoustics …………..................................... 8

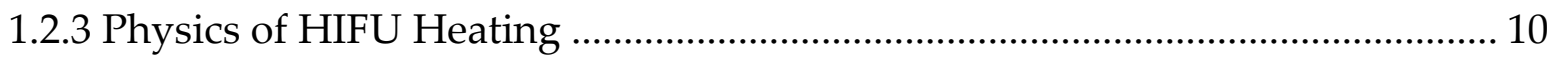

1.2.4 Numerical Methods for Solving the 2-Dimensional Heat Equation .................. 11

1.3 Effect of Perfusion on HIFU Ablation ....................................................................... 15

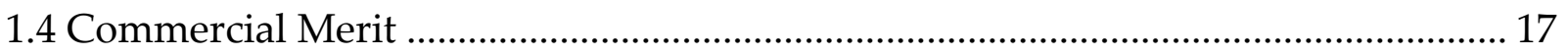

Chapter Two Related Work ............................................................................................. 19

2.1 HIFU for Ablation of Breast Tumors ...................................................................... 19

2.2 Modeling of Perfused Tissue and HIFU Ablation .................................................... 21

2.2.1 Pennes' Bioheat Transfer Equation ...................................................................... 21 
2.2.2 "Enhanced" Tissue Conductivity Model.

2.2.3 Varying Vessel Convection Model ....................................................................... 24

2.2.4 Modeling Ultrasound Heating in Media .............................................................. 26

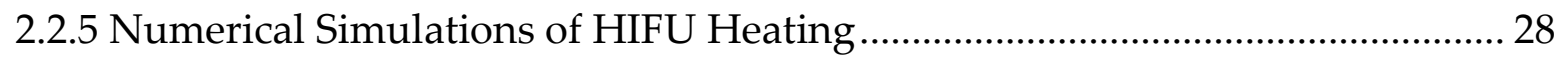

2.3 Tissue-Mimicking Materials (TMMs) for HIFU Ablation ........................................... 30

2.4 Research on the Effects of Blood Vessels on HIFU Ablation ...................................... 33

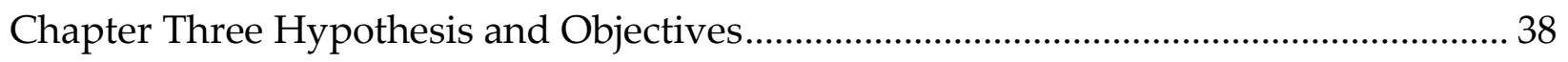

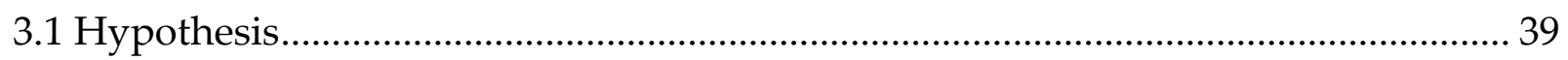

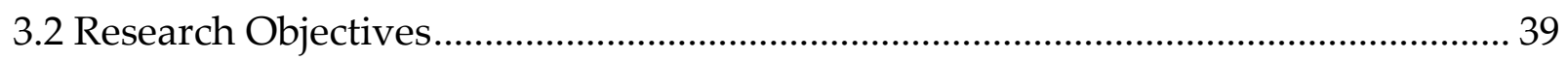

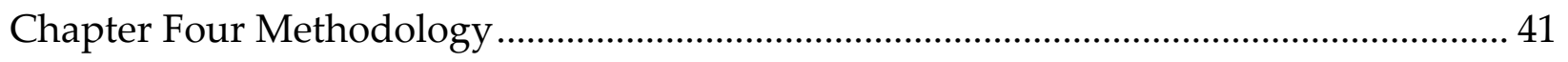

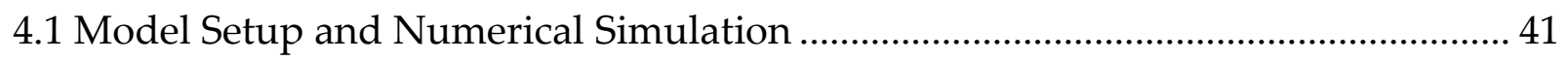

4.1.1 Physics Modeling and Assumptions...................................................................... 41

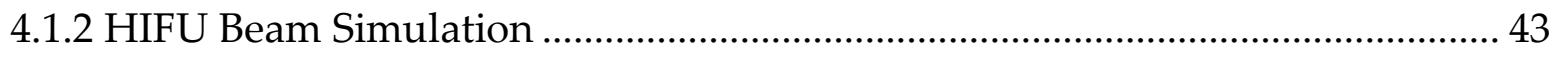

4.1.3 Numerical Simulation of Heat Transfer ............................................................... 46

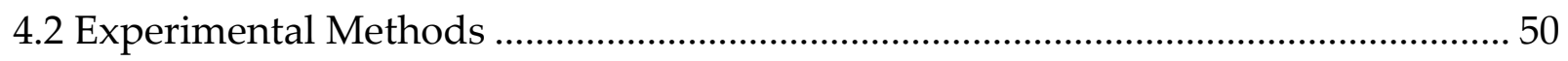

4.2.1 Tissue Phantom Materials and Preparation........................................................ 51 


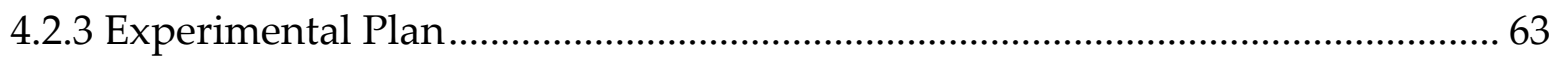

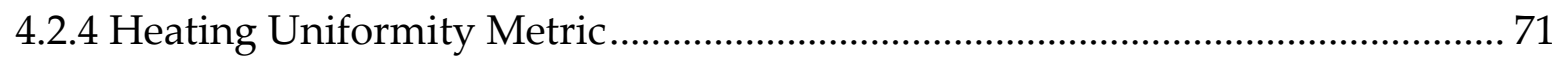

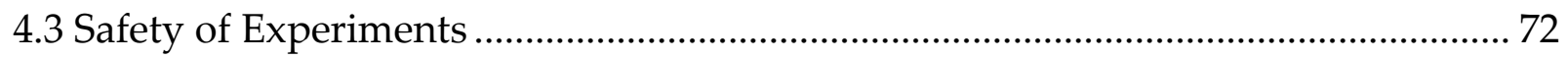

Chapter Five Results and Discussion............................................................................... 74

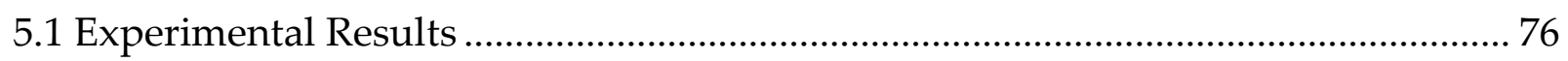

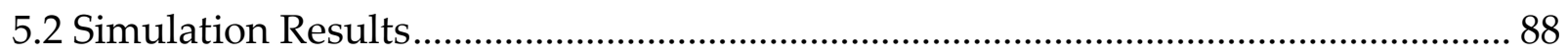

Chapter Six Conclusions and Future Work …………....................................................... 97

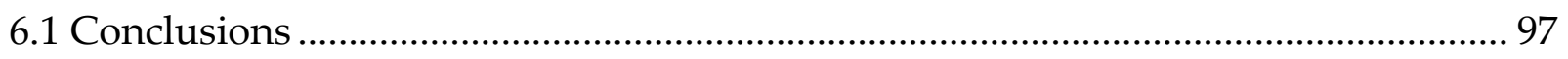

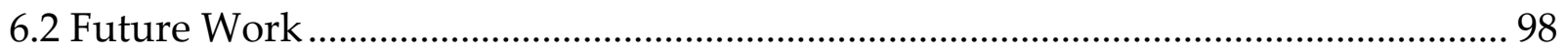

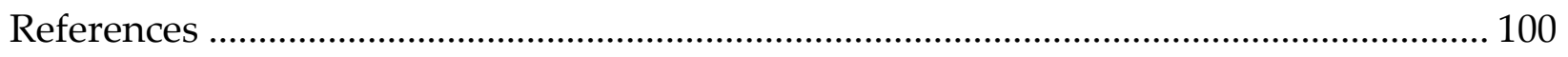

Appendix A HIFU Simulation MATLAB Script............................................................... 107

Appendix B Temperature Plotting MATLAB Script ………............................................. 116 


\section{LIST OF FIGURES}

Figure 1. HIFU pressure wave and heating characteristics 5

Figure 2. Simulated effect of blood vessel (a) on tissue heating symmetry and (b) change in blood temperature during heating 16

Figure 3. Illustrations of (a) thermal lesion formation and (b) consecutive lesion formations 20

Figure 4. HIFU simulation 21

Figure 6. Use of CT/MRI images for HIFU model 28

Figure 7. Plots of simulated (a) heating rate, (b) temperature profile in tissue .... 29 Figure 8. Side-view of a polyacrylamide/BVA vessel phantom with a circumferential lesion made by ultrasound 32

Figure 9. HIFU phantom with vessel and embedded thermocouples 34

Figure 10. (a) Tissue/vessel geometry and (b) temperatures with and without

vessels

Figure 11. (a) Thermal dose mid-plane with fixed power and (b) with variable

power 36

Figure 12. (a) Intensity and (b) heating rate of HIFU transducer at $30 \mathrm{~W}$ 45

Figure 13. Example of interior node and energy balance 47

Figure 14. Experimental setup showing the phantom and HIFU fixtures 51 ix 
Figure 15. Equipment used for phantom fabrication................................................ 53

Figure 16. Phantom mold with thermocouple fixture (a) before TMM is added and (b) after addition of TMM ............................................................................ 54

Figure 17. Reservoir and flow system setup .......................................................... 57

Figure 18. H-101 Transducer and power system.................................................... 58

Figure 19. Tri-axis motor system and water tank setup .......................................... 60

Figure 20. Compression arm, linear rail and micro-controller.................................. 61

Figure 21. Thermocouple spacing relative to HIFU focus (in red) ......................... 62

Figure 22. Fixture with thin-wire thermocouples inside sheathed hypo-tubes.... 63

Figure 23. Ultrasound power meter for measuring HIFU transducer output ...... 64

Figure 24. (a) Illustration of HIFU focus adjustment after compression and (b) example of reflected ultrasound imaging waveform ............................................. 67

Figure 25. Plot of acquired temperature data at various depths in phantom ....... 70

Figure 26. Relationship between displacement of phantom wall and flow rate .. 76 Figure 27. Thermocouple locations relative to the HIFU focal point and vessel.. 77 Figure 28. Raw temperature plots at each thermocouple for all flow rates 82

Figure 29. Transient temperature plots at $+2 \mathrm{~mm}$ for flows between (a) 40-20 $\mathrm{mL} / \mathrm{min}$ and (b) $20-0 \mathrm{~mL} / \mathrm{min}$ 83

Figure 30. Plot of flow rate vs. compression of the tissue-mimicking material.... 84 
Figure 31. Maximum temperatures at $+2 \mathrm{~mm}$ (side nearest fluid vessel) as a

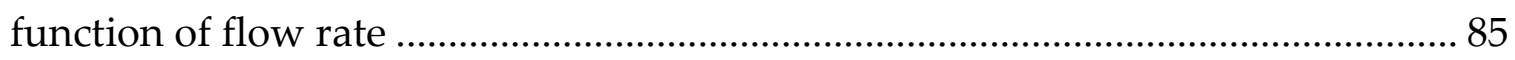

Figure 32. Comparison of maximum temperatures at $+2 \mathrm{~mm}$ and $-2 \mathrm{~mm}$ as a

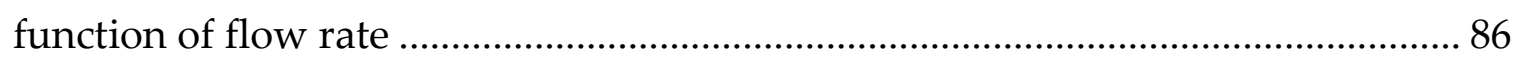

Figure 33 (a) Simulated temperature profile, 30 seconds of heating, no flow ...... 89

Figure 34 (a) Simulated temperature profile with $40 \mathrm{~mL} / \mathrm{min}$ of flow ................... 90

Figure 35 (a) Temperature profile and (b) 1-D radial plot after 30 sec of heating 92 


\section{LIST OF TABLES}

Table 1. Peak temporal average intensities of ultrasound sources .......................... 6

Table 2. Properties used in model ...................................................................... 42

Table 3. Thermal properties for heat transfer simulation ......................................... 49

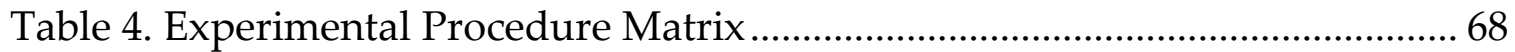

Table 5. Measurement of Simulated Uniformity Metrics............................................. 94 


\section{CHAPTER ONE \\ INTRODUCTION}

Breast cancer is the most commonly diagnosed cancer in women with the exception of skin cancer. According to the American Cancer Society (2012), more than 230,000 women in the United States were diagnosed with invasive breast cancer in 2011 [1]. Some common treatment methods are chemotherapy and radiotherapy, both of which have serious adverse side-effects on patients. Hyperthermia has been used as an adjuvant therapy that has synergistic effects when used with chemo- or radiotherapy [2]. High temperature hyperthermia has also been used as a stand-alone therapy for ablation of tumors.

\subsection{Introduction to Cancer Treatment and HIFU Therapy}

Hyperthermia therapy uses temperatures above standard body temperature_levels to change the properties of the targeted tissue. At temperatures of $38-43^{\circ} \mathrm{C}$, normal tissue experiences increased blood flow and increased metabolism. At temperatures of $43-50^{\circ} \mathrm{C}$, tumors experience increased blood flow and oxygenation, inhibited radiation repair, vascular stasis, DNA damage, and increased sensitivity to radiotherapy. At temperatures above $50^{\circ} \mathrm{C}$, 
tissue experiences protein coagulation, ablation, and vaporization resulting in immediate cell death [3].

Some of the methods used to achieve hyperthermia in tumors are ultrasound (US), electromagnetic radiation, specifically microwave (MW) and radiofrequency $(\mathrm{RF})$, and conduction from heat sources such as hot water tubes. These hyperthermia therapies generally can be applied externally or interstitially. External therapies are essentially non-invasive methods where a tumor can be heated by an applicator outside of the breast. Interstitial therapies use a catheter to insert antennas or tubing so that the origin of the heat source is inside the tumor. External hyperthermia eliminates the need for invasive operations, yet interstitial hyperthermia allows for isolated local treatment that minimizes the effects on the rest of the body [3].

Hyperthermia treatment can be split into two major categories: 1) mild hyperthermia used as adjuvant therapy, and 2) high-temperature hyperthermia meant to cause tumor tissue necrosis. Mild hyperthermia is generally considered to be the heating of tissue to between $37-50^{\circ} \mathrm{C}$, whereas high-temperature hyperthermia heats tissue to temperatures above $50^{\circ} \mathrm{C}$ (can be greater than $100^{\circ} \mathrm{C}$ ) [3]. Song et al. [4] state that when mild hyperthermia is applied to tumors, blood flow and oxygenation increase, resulting in more effective chemo- 
and radiotherapy. Numerous phase-III trials have found that combined radiotherapy and hyperthermia treatment of tumors results in improved treatment response and survival rate for patients [5]. At temperatures above $50^{\circ} \mathrm{C}$, the targeted tissue experiences protein denaturation and tissue necrosis [3].

Thermal ablation essentially works by raising the temperature in tissue beyond the point of cellular necrosis, effectively killing the cancerous cells within the targeted region, or region of interest (ROI). HIFU, applied as a non-invasive, targeted and radiation-free procedure, has been shown to effectively ablate cancerous tissue in the breast [6]. HIFU technology has received much attention since researchers first created thermal lesions in animal tissues in the 1970s and 1980s [7]. Clinical trials have been conducted or planned to investigate HIFU treatment of prostate, breast, liver, brain, and kidney cancer, as well as treatment of uterine fibroids and other pathological conditions $[7,8]$. HIFU technology is relatively simple, safe, and inexpensive and has the potential to be an important tool for treatment of many diseases and medical issues.

\subsection{HIFU Physics and Transducer Design}

Ultrasound transducers are, at a basic level, membranes that vibrate to produce pressure waves at frequencies above $16 \mathrm{kHz}$, which is the upper limit of human hearing. Medical ultrasound transducers are generally designed to 
operate from $1-20 \mathrm{MHz}(1 \mathrm{MHz}=1,000 \mathrm{kHz})$ [7]. The vibrations are generated by quartz crystals, called piezoelectric crystals, which vibrate when introduced to a alternating current source. This physical phenomenon, discovered by Jacques and Pierre Curie in the late 1800s, is called the piezoelectric effect [9]. Transducers can have multiple piezoelectric crystals arranged in an array, which can be controlled by a power supply and function generator. Different piezoelectric materials have been used for ultrasound transducers; the most common being lead zirconate titanate (PZT) crystals [10].

The concept of HIFU transducers is based on the fact that sound waves are additive in nature and can therefore be combined at a focal point to create a wave with greater amplitude than the individual components. Precisely shaped transducer arrays can be designed to produce a high-energy focal point described by its focal length, the location where the ultrasound waves coming from the individual transducer elements meet. The energy of each individual ultrasound wave is low enough to avoid significantly affecting the tissue it passes through, but the intensity at the focal point is great enough to cause coagulative necrosis [7]. Figure 1 illustrates the additive nature of focused ultrasound waves. 


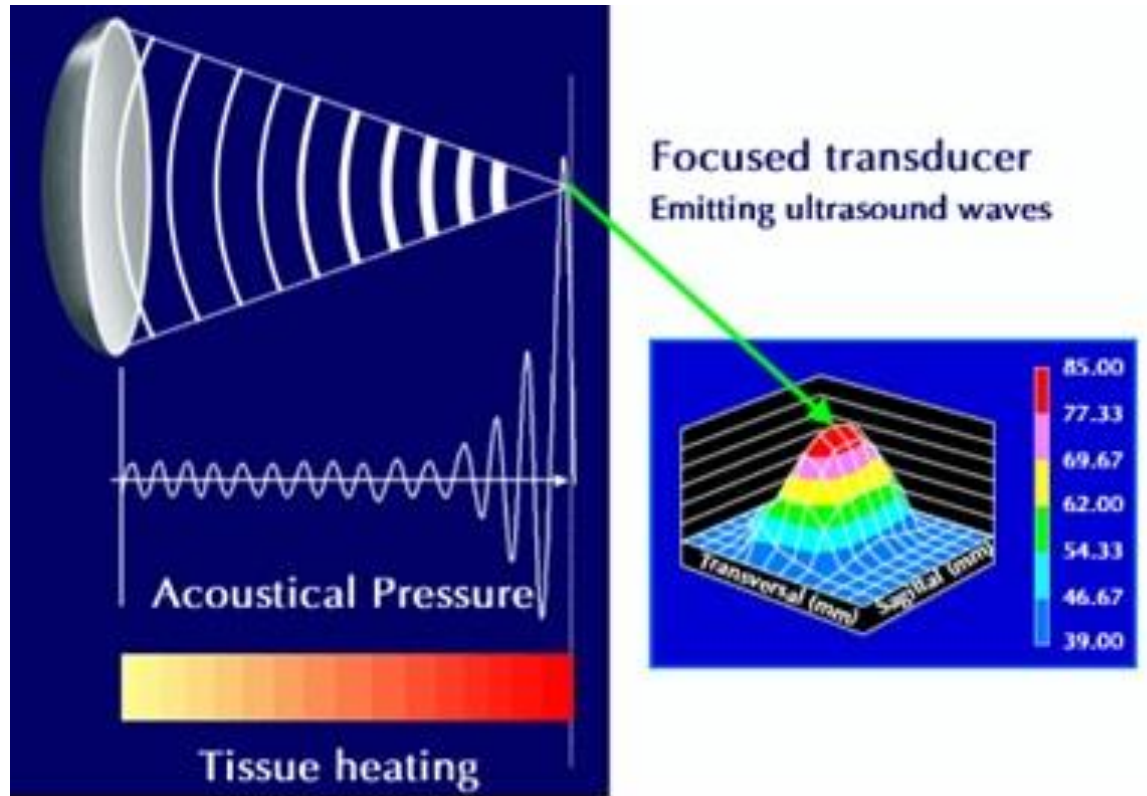

Figure 1. HIFU pressure wave and heating characteristics reprinted with permission from Maple Leaf HIFU [11]

Device companies have taken advantage of this concept to apply high ultrasound energies to targeted regions, at a distance from the ultrasound source. As a non-invasive, targeted treatment option, HIFU is very attractive for many applications.

\subsubsection{Ultrasound Wave Propagation through Media}

HIFU sources can be divided into component pressure waves that propagate through a medium. In elastic media, such as soft tissue, the particles of the medium vibrate as a result of the oscillations of the ultrasonic waves. 
Humphrey [12] denotes the particle displacement as $\xi$ and describes the particle velocity $u$ and acceleration $a$ as

$$
\begin{aligned}
& \vec{u}=\frac{\partial \xi}{\partial t} \\
& \vec{a}=\frac{\partial \vec{u}}{\partial t}
\end{aligned}
$$

When the particles oscillate and interact, the density $\left(\rho_{0}\right)$ and pressure $(p)$ of the medium experience local changes. The pressure-particle velocity relationship is

$$
p=\rho_{0} c_{0} u
$$

Equation 3

where $c_{0}$ is the speed of sound through the medium.

Acoustic energy of a plane wave can be characterized by its delivery rate through the medium, referred to as the intensity (I) [12].

$$
I=p^{2} / 2 \rho_{0} c_{0}
$$

Equation 4

The pressure amplitude of the acoustic wave is denoted as $p$. Some common

\begin{tabular}{|c|c|c|c|}
\hline Modality & Frequency (MHz) & $\begin{array}{c}\text { Peak rarefactional } \\
\text { pressure (MPa) }\end{array}$ & $I_{\text {SPTA }}\left(\mathrm{W} \mathrm{cm}^{-2}\right)$ \\
\hline B-mode & $1-15$ & $0.45-5.5$ & $0.0003-0.99$ \\
\hline
\end{tabular}
intensity values for HIFU and other ultrasound modes are shown in Table 1, where ISPTA is peak temporal average intensity.

Table 1. Peak temporal average intensities of ultrasound sources [12] 


\begin{tabular}{|c|c|c|c|}
\hline $\begin{array}{c}\text { Pulse-Width } \\
\text { Doppler }\end{array}$ & $1-10$ & $0.67-5.3$ & $0.17-9.1$ \\
\hline Physiotherapy & $0.75-3.4$ & 0.3 & 3 \\
\hline HIFU & $1-10$ & & $\mathbf{1 , 0 0 0 - 1 0 , 0 0 0}$ \\
\hline Lithotripsy & $0.5-10$ & $5-15$ & \\
\hline
\end{tabular}

As is evident from Table 1, HIFU sources can supply peak intensities much higher than the other ultrasound modalities used in medicine. These large intensity values are the reason that HIFU sources can ablate tissue. Ultrasound energy is absorbed and reflected as it passes through a medium such as soft tissue. The amounts of absorption and reflection that occur depend on the material properties and the ultrasound intensity. Local HIFU intensity is defined as,

$$
I=I_{0} e^{-2 \propto_{a} z},
$$

Equation 5

where $I_{0}$ is the initial intensity of the wave, $\propto_{a}$ is the absorption coefficient of the medium and $z$ is the distance the wave has traveled in the direction of propagation [12]. 


\subsubsection{Equations for Modeling Nonlinear Acoustics}

Linear approximations of ultrasound wave propagation have been developed and used in many models, but the physical phenomenon includes nonlinear effects. The nonlinear aspects of ultrasound propagation can be pronounced in various biomedical applications such as HIFU and ultrasound imaging due to the frequency and intensity ranges involved [13]. This section reviews the governing equations used to model ultrasound propagation with the inclusion of nonlinear effects.

The Lossless Theory assumes that entropy remains constant in the system and that pressure will only depend on density [13]. The wave equation for a plane wave, with the application of the Lossless Theory, is

$$
\frac{\partial q}{\partial t}+\left(c_{0}+\beta v\right) \frac{\partial q}{\partial x}=0
$$

where $q$ is the acoustic pressure $\left(P-P_{0}\right)$ or excess density $\left(\rho-\rho_{0}\right), c_{0}$ is the small-signal speed of sound, $v$ is the particle velocity, and $\beta=1+\mathrm{B} / 2 \mathrm{~A}$ is the coefficient that determines the speed of sound through a medium and accounts for nonlinearity. The ratio $B / A$ is defined as follows.

$$
\frac{B}{A}=\left.\frac{\rho_{0}}{c_{0}^{2}} \frac{\partial^{2} p}{\partial \rho^{2}}\right|_{0}
$$


Implicit solutions exist for Equation 7 for various initial value and boundary value problems (i.e. $v(x, 0)=G(x))$ [13]. If $\frac{\beta v}{c_{0}} \ll 1$ and a retarded time variable is used $\left(\tau=t-x / c_{0}\right)$, Equation 7 can be reduced to,

$$
\frac{\partial v}{\partial x^{\prime}}=\frac{\beta v \partial v}{c_{0}^{2} \partial \tau}
$$

Burgers' equation takes the wave equation (with lossless assumptions) with propagation through a dissipative medium, and assume "weak absorption due to viscosity and heat conduction." [13] Assuming that the dominant factor in dissipation is the viscous loss in the medium, the plane wave equation becomes,

$$
\frac{\partial v(z, \tau)}{\partial x}=\frac{\beta v \partial v}{c_{0}{ }^{2} \partial \tau}+\frac{1}{2 c_{0}^{3} \rho_{0}}\left(\mu_{B}+\frac{4}{3} \mu\right) \frac{\partial^{2} v}{\partial \tau^{2}} \quad \text { Equation } 9
$$

where $\mu$ is the coefficient of shear viscosity and $\mu_{B}$ the coefficient of bulk viscosity. Burgers' equation was built upon to develop a three dimensional system of wave equations [13].

The three-dimensional wave equation, which considers diffraction, absorption and nonlinearity effects, was developed by Kuznetsov, Zabolotskaya and Khokhlov (KZK) [13]. The KZK equation can be written as,

$$
\frac{\partial^{2} p}{\partial \tau \partial z}-\frac{c_{0}}{2} \nabla^{2}{ }_{\perp} p=\frac{1}{2 c_{0}{ }^{3} \rho_{0}} \frac{\partial}{\partial \tau}\left[\left(\mu_{B}+\frac{4}{3} \mu\right) \frac{\partial^{2} p}{\partial \tau^{2}}+\beta \frac{\partial p^{2}}{\partial \tau}\right]
$$

where $\nabla_{\perp}^{2}$ is the transverse Laplacian and $\tau=t-z / c_{0}$. The second term on the left hand side accounts for diffraction of the wave. The first term in parentheses 
on the right hand side represents the effects of thermoviscous attenuation, while the second term accounts for nonlinear effects (notice the presence of the nonlinear coefficient, $\beta$ ). [13] The KZK equation is used in many HIFU simulations.

\subsubsection{Physics of HIFU Heating}

Acoustic energy is absorbed as ultrasonic waves propagate through a medium. This results in heating of the medium, which can be represented by a heat rate per unit area or volume. [13]

$$
q_{a b s}=2 \propto_{a} I,
$$

Equation 11

where $I$ is the local wave intensity and $\propto_{a}$ is the absorption coefficient for the medium. This energy is delivered into the medium and most of it is converted into heat. For a solid medium such as soft tissue, the standard heat equation with heat generation can often be used to solve for the steady-state or transient temperature during HIFU heating. The transient heat equation with an energy source can be written as,

$$
\rho c_{p} \frac{\partial T}{\partial t}=\nabla \cdot(k \nabla T)+q_{a b s}
$$

Equation 12 
where the partial derivative is the time-dependent change in temperature $T, \rho$ is the material density, $c_{p}$ is the specific heat of the medium, and $\nabla$ is the Laplacian operator [13].

In most soft tissues, there is additional heat transfer that occurs because of local blood perfusion and metabolic processes. The governing equations for perfused tissues are more complex and numerous models have been proposed, each with its own assumptions and simplifications. These models are discussed in more detail in Chapter 2.

\subsubsection{Numerical Methods for Solving the 2-Dimensional Heat Equation}

Engineering problems that feature thermal conduction and energy deposition are often solved with numerical methods and computers. Finite element (FE), finite difference (FD), and finite volume (FV) approaches are the most common methods found in the literature for solving heat transfer problems. These methods discretize the heat equation and the material geometry, dividing it into fixed-size elements. The most convenient and straight-forward method for this investigation is the FD method, which can easily be implemented using numerical computation software and custom written code.

To solve a heat transfer problem with the FD method, the heat equation (Equation 12) must first be rewritten in FD form. There are various approaches 
for setting up and solving this equation, including explicit, implicit, and hybrid methods. The explicit form [14], which solves for unknown temperature nodes using know nodal temperatures, is shown below.

$$
\begin{gathered}
\rho c_{p} \frac{\left(T^{t+1}{ }_{i, j}-T^{t}{ }_{i, j}\right)}{\Delta t}= \\
k\left[\frac{T_{i-1, j}^{t}-2 T^{t}{ }_{i, j}+T^{t}{ }_{i+1, j}}{(\Delta x)^{2}}+\frac{T^{t}{ }_{i, j-1}-2 T^{t}{ }_{i, j}+T^{t}{ }_{i, j+1}}{(\Delta y)^{2}}\right]+q_{a b s}
\end{gathered}
$$

where $\Delta t$ is the finite time-step size, $t$ is the known step in time, $t+1$ is the proceeding step in time, $i$ is the location of the node on the horizontal axis, $j$ is the location of the node on the vertical axis, $\Delta x$ is the finite step-size in the horizontal direction and $\Delta y$ is the finite step-size in the vertical direction. This approach provides just one unknown $\left(T^{t+1}{ }_{i, j}\right)$, which can be solved relatively easily.

This approach offers a simple, easy to solve equation for each material node at each time step, but it also has a stability limitation. The calculated values of $T^{t+1}{ }_{i, j}$ are only valid if the Courant-Friedrichs-Lewy (CFL) condition is met [15]. The CFL condition requires that,

$$
\frac{k}{\rho c_{p}} \Delta t \leq \frac{1}{2} \frac{\Delta x^{2} \Delta y^{2}}{\Delta x^{2}+\Delta y^{2}}
$$


If the CFL condition is too limiting for the problem being solved, an implicit method can be used instead. The implicit method solves multiple temperature nodes at the new time step, a method called backward-difference approximation. This approach gives the following 2-D equation:

$$
\begin{array}{cc}
\rho c_{p} \frac{\left(T^{t+1}{ }_{i, j}-T_{i, j}^{t}\right)}{\Delta t}=k\left[\frac{T^{t+1}{ }_{i-1, j}-2 T^{t+1}{ }_{i, j}+T^{t+1}{ }_{i+1, j}}{(\Delta x)^{2}}+\right. & \text { Equation } 15 \\
\left.\frac{T_{i, j-1}^{t+1}-2 T^{t+1}{ }_{i, j}+T^{t+1}{ }_{i, j+1}}{(\Delta y)^{2}}\right]+q_{a b s} &
\end{array}
$$

The implicit method unconditionally stable, but becomes inaccurate when using large time-steps $(\Delta t)[14]$.

The final approach considered in this review is called the Crank-Nicolson (CN) method. The $\mathrm{CN}$ method is a combination of the implicit and explicit methods. Weighted averaging is used to arrive at the following equation for the 1-D heat equation, which solves for the temperature at node $(i, j)$ at time $(t+1)$ :

$$
\begin{gathered}
T_{i}^{t+1}=T_{i}^{t}+\frac{\alpha \Delta t}{2(\Delta x)^{2}}\left[\left(T_{i-1}{ }^{t}-2 T_{i}{ }^{t}+T_{i+1}{ }^{t}\right)+\left(T_{i-1}{ }^{t+1}-\quad \text { Equation } 16\right.\right. \\
\left.\left.2 T_{i}{ }^{t+1}+T_{i+1}{ }^{t+1}\right)\right]
\end{gathered}
$$

Equation 16 is considered "semi-implicit" and can be solved with various techniques, such as the Thomas algorithm [14]. By arranging the unknown values on the left side of the equation and the known values on the right, the equation becomes, 


$$
\begin{gathered}
(r+1) T_{i}^{t+1}-\frac{r}{2}\left(T_{i-1}{ }^{t+1}+T_{i-1}{ }^{t+1}\right) \\
=T_{i}^{t}+\frac{r}{2}\left(T_{i-1}{ }^{t}-2 T_{i}^{t}+T_{i+1}^{t}\right)
\end{gathered}
$$

where $r=\alpha \Delta t /(\Delta x)^{2}$. This formula can be converted into matrix form that can be solved using computational software. The matrix form, $\boldsymbol{A} * \overline{\boldsymbol{u}}^{\boldsymbol{n}+\boldsymbol{1}}=\boldsymbol{B} \overline{\boldsymbol{u}}^{\boldsymbol{n}}$, is as follows:

$$
\begin{gathered}
{\left[\begin{array}{cccccc}
2(r+1) & -r & 0 & \cdots & \cdots & 0 \\
-r & 2(r+1) & -r & 0 & \cdots & \vdots \\
0 & -r & 2(r+1) & -r & 0 & \vdots \\
\vdots & \ddots & \ddots & \ddots & \ddots & \vdots \\
0 & \cdots & \cdots & 0 & -r & 2(r+1)
\end{array}\right]\left[\begin{array}{c}
T_{2}{ }^{t+1} \\
\vdots \\
T_{n x-1}{ }^{t+1}
\end{array}\right]} \\
=\left[\begin{array}{c}
2 T_{2}{ }^{t}+r\left({T_{1}}^{t}-2{T_{2}}^{t}+T_{3}{ }^{t}\right)+r\left(T_{1}{ }^{t+1}\right) \\
2 T_{3}{ }^{t}+r\left(T_{2}{ }^{t}-2 T_{3}{ }^{t}+T_{4}{ }^{t}\right) \\
\vdots \\
2 T_{n x-2}{ }^{t}+r\left(T_{n x-3}{ }^{t}-2 T_{n x-2}{ }^{t}+T_{n x-1}{ }^{t}\right) \\
2 T_{n x-1}{ }^{t}+r\left(T_{n x-2}{ }^{t}-2 T_{n x-1}{ }^{t}+T_{n x}{ }^{t}\right)+r\left(T_{n x}{ }^{t+1}\right)
\end{array}\right]
\end{gathered}
$$

Equation 18

where $T_{1}{ }^{t+1}$ and $T_{n x}{ }^{t+1}$ are the temperatures defined by the boundary conditions.

This system of equations can be solved by taking the inverse of $\boldsymbol{A}$ and multiplying it by $\boldsymbol{B}$.

$$
\left[\begin{array}{c}
T_{2}{ }^{t+1} \\
\vdots \\
T_{n x-1}{ }^{t+1}
\end{array}\right]=\boldsymbol{A}^{-1} \boldsymbol{B}
$$

The CN method is unconditionally stable, but it is subject to "severe oscillations" under certain boundary conditions [16]. 
All of the methods reviewed in this section can be implemented using commercial computational software such as MATLAB (The MathWorks, Inc., Natick, Massachusetts, USA). The choice between methods should be based on the required or desired computation speed and space and time step sizes being used.

\subsection{Effect of Perfusion on HIFU Ablation}

Non-invasive HIFU ablation treatment has many characteristics that make it desirable for the treatment of cancer, yet there are issues that have prevented it from becoming an effective and competitive method. Blood flow through vessels in the vicinity of the targeted tumors is the main source of (convective) heat transfer away from the region, essentially acting as a heat sink that affects the uniformity and peak temperature of the hyperthermic region. Figure 2 shows a simulation of tissue heating near a vessel, including temperature plots of the tissue region and the blood flow region separately. 


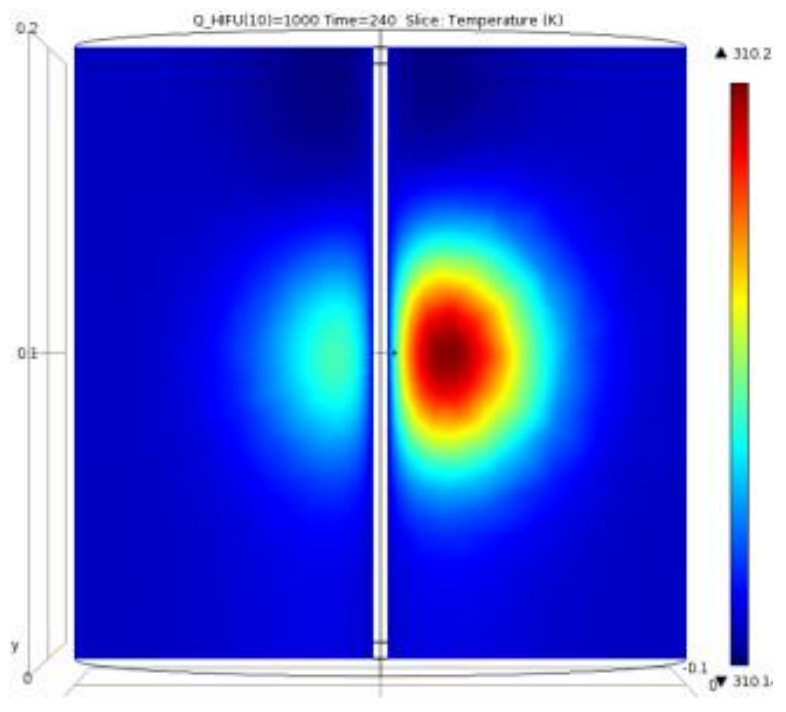

(a)

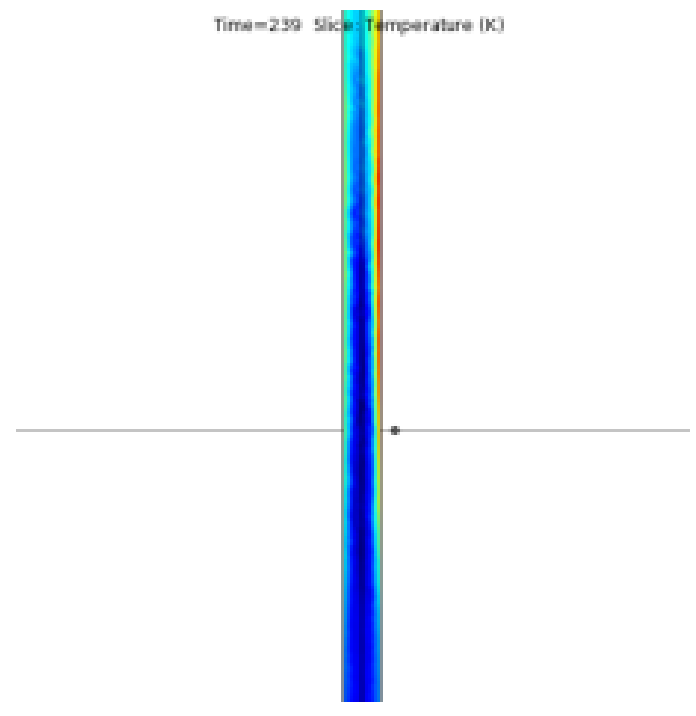

(b)

Figure 2. Simulated effect of blood vessel (a) on tissue heating symmetry and (b) change in blood temperature during heating

Studies by Dasgupta et al. [17], have found that convective heat transfer due to blood vessels can decrease temperatures by more than $10 \%$ in the region close to the HIFU focus. Other studies found that "about 10 times as much power" was needed to maintain the proper hyperthermic temperature in a real breast compared to a non-perfused tissue-mimicking material, suggesting the significance of heat transfer due to blood perfusion [18]. Huang et al. [19] found that with $2 \mathrm{~cm} / \mathrm{s}$ of flow through a phantom vessel, peak temperature during HIFU heating was reduced by $25 \%$. The thermal effects of perfusion depend on the size and number of vessels in the target tissue region. One study defines 
vessels with diameters from $2 \mathrm{~mm}$ to $5 \mathrm{~mm}$ as "thermally significant," meaning that vessels in this range significantly affect heat transfer during HIFU ablation [20].

Vasculature plays an important role in the heating characteristics of tissues and tumors. The convective heat transfer that occurs in the vicinity of

thermally significant blood vessels can make HIFU treatment predictability (and therefore treatment planning) difficult in a clinical setting. To ensure that a tumor is completely ablated in a perfused tissue region, higher HIFU powers and/or durations would be required, resulting in a higher risk of tissue damage during ablation.

\subsection{Commercial Merit}

Presently HIFU ablation is a limited technique for treating cancer, but it has the potential to become a mainstream treatment. The global market for cancer treatment has been estimated around $\$ 78$ billion for 2012. The market for ablation devices in the United States is estimated at $\$ 730$ million alone [21]. The future prospects of the market are positive, with expectations of "moderate to very healthy compound annual growth - ranging from 3.3\%-17.7\% - due to strong demand, cost-efficiency and efficacy, strong reimbursement, emerging 
unique, proprietary cost-effective, multi-platform next-generation technologies, and an aging population susceptible to various diseases/conditions." [22] 


\section{CHAPTER TWO \\ RELATED WORK}

In response to the potential benefits of HIFU tumor ablation, much effort has been invested in research and development of the technology. A wide array of subtopics have been studied, including physiological effects of HIFU ablation, tissue-heating characteristics of HIFU transducers, effects of blood perfusion on heating profiles, treatment modeling and prediction, and the development of tissue-mimicking materials (tissue phantoms) for simulating HIFU tissue ablation

\subsection{HIFU for Ablation of Breast Tumors}

HIFU is a promising treatment option for the ablation of breast tumors due to its non-invasive nature. As mentioned previously, HIFU can be used to increase the temperature in tissue until coagulative necrosis occurs. This generally occurs at temperatures at or above $55^{\circ} \mathrm{C}$, although temperatures at the center of the focal volume can reach in excess of $80{ }^{\circ} \mathrm{C}$ [6]. When HIFU is focused on a breast tumor, a large temperature gradient exists along the periphery of the thermal lesion being formed. This results in lethal temperatures within the focal volume and safe temperatures outside of it, protecting the surrounding tissues in the breast. In addition to coagulative necrosis of cancerous cells in the breast, 
HIFU can be used to sever nutrient- and oxygen-delivery vessels that feed the tumor [6]. Figure 3 illustrates the formation of a thermal lesion formed by HIFU.

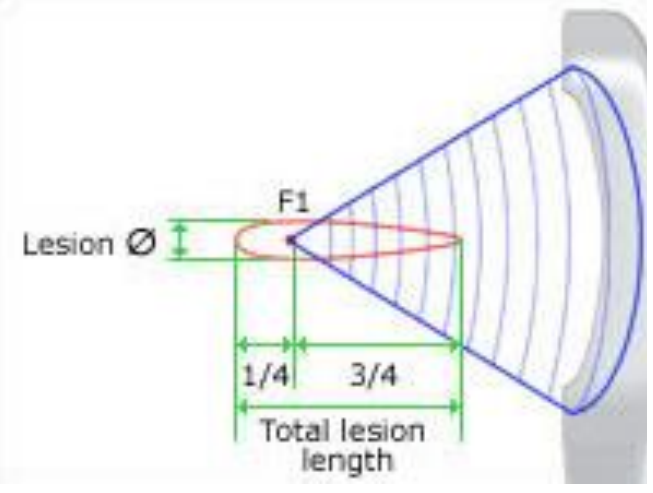

(a)

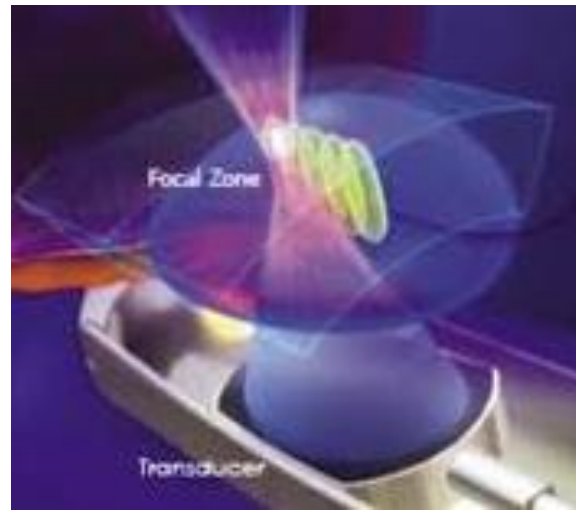

(b)

Figure 3. Illustrations of (a) thermal lesion formation and (b) consecutive lesion formations, reprinted with permission from Maple Leaf HIFU [11]

Some existing HIFU devices being used to treat breast cancer include the ExAblate 2000 (InSightec-TxSonics Ltd.) and the JC-HIFU system (Haifu Technology Co. Ltd.), commercial packages being sold outside the U.S. The ExAblate 2000 uses magnetic resonance imaging (MRI) to guide the ablation treatment and monitor the real-time temperature of the tissue. MRI thermometry can be a useful tool during ablation, but the "frequency shifting" technique used by the ExAblate does not work well with fatty tissues such as those found in the breast. The JC-HIFU uses ultrasound guidance, which can provide fast and cheap imaging compared to MRI, but tissue damage has proven to be difficult to assess with this method unless acoustic cavitation occurs [6]. 
2.2 Modeling of Perfused Tissue and HIFU Ablation

Due to the complex nature of HIFU modeling, as well as the increased computational capabilities of personal computers, numerical simulations have become a valuable and widely used tool in the biomedical field. The physics of ultrasound propagation, interaction with media, and tissue heating are well understood and a lot of models have been created for device and treatment development purposes. Figure 4 shows an example of a multi-element HIFU transducer simulation and highlights the capabilities of computer simulations in modeling HIFU beam production, propagation and absorption.

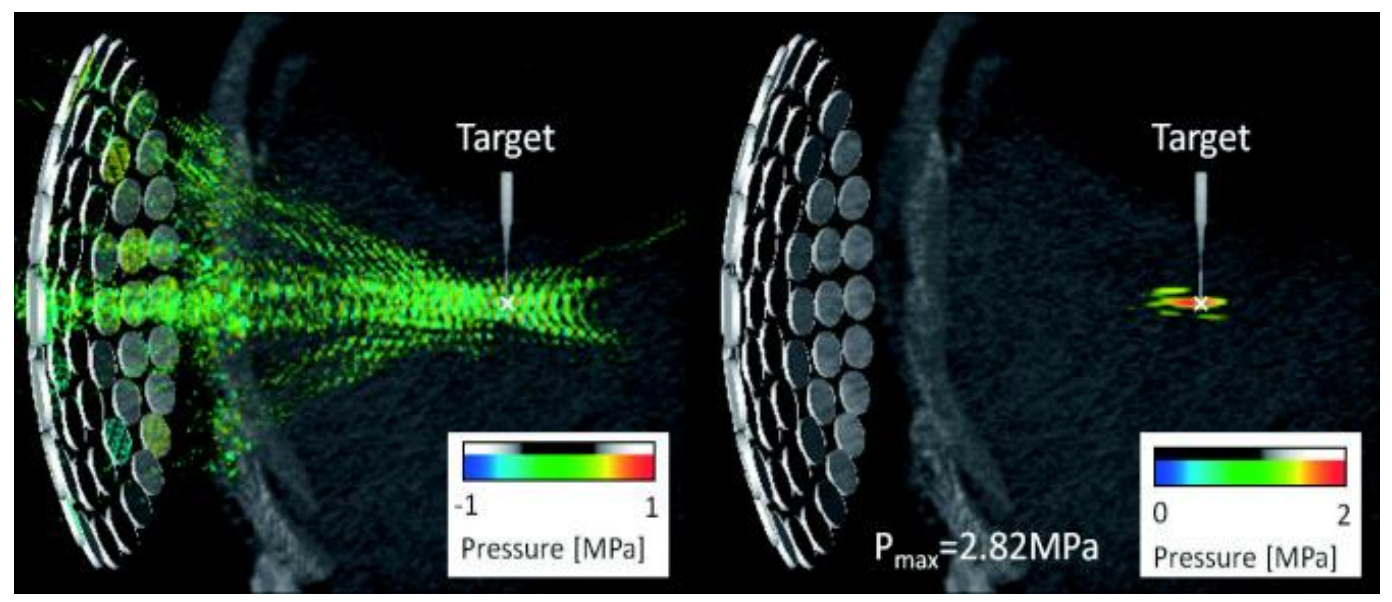

Figure 4. HIFU simulation, reprinted with permission from Okita et al. [23]

\subsubsection{Pennes' Bioheat Transfer Equation}

The most widely-known and utilized mathematical model for transient heat transfer in tissues was developed by Harry Pennes in the late 1940s. Pennes' model equation, called the bioheat transfer equation (BHTE) [24], features a 
uniform heat-sink term based on average blood-perfusion in the tissue. The general representation of the BHTE is

$$
\rho_{t} c_{t} \frac{\partial T}{\partial t}=k \nabla^{2} T-\omega_{b} c_{b}\left(T-T_{a}\right)+q_{a b s}
$$

where $\rho_{\mathrm{t}}$ is the density of the tissue, $\mathrm{c}_{\mathrm{t}}$ is the specific heat of the tissue, $\mathrm{T}$ is the tissue temperature, $\mathrm{k}$ is the thermal conduction coefficient, $\omega_{\mathrm{b}}$ is the blood perfusion rate, $c_{b}$ is the specific heat of blood, $T_{a}$ is the initial inlet temperature of the blood (approximated as the arterial blood temperature), and $q_{a b s}$ is the energy generated by metabolic or external sources. In the situation of HIFU ablation, it can be assumed that the metabolic energy generation is much smaller than the ultrasonic energy generation and can therefore be ignored (this is the case when large energies are generated by any outside source).

While there is "good agreement" between the BHTE and experimental data [13], further research found that the BHTE is not sufficient in describing the heat transfer in tissues when "thermally significant" blood vessels are present. Several models that take these thermally significant vessels into account have been proposed [19]. Rawnsley et al. [25] included the effects of small numbers of blood vessel pairs (artery/vein) in addition to the general BHTE, finding that the modeled correlated much better with experimental results. 


\subsection{2 “Enhanced” Tissue Conductivity Model}

Chen and Holmes developed another popular mathematical model [20] consisting of a solid tissue region and a fluid (blood) region, each governed by the appropriate energy balance equations. According to Narasimhan [26], this model acts like a classic "porous medium." The model equation by Chen and Holmes is,

$$
\begin{gathered}
(\rho c)_{t} \frac{\partial T_{t}}{\partial t}=k_{t} \nabla^{2} T_{t}+k_{p} \nabla^{2} T_{t}+V_{j}^{m}\left(\rho c_{p}\right)_{b} *\left(T_{a}-T_{t}\right)-\quad \text { Equation } 21 \\
\left(\rho c_{p}\right)_{b} \vec{v} \cdot \nabla T_{t}+Q_{m}^{\prime \prime \prime}
\end{gathered}
$$

where $k_{p}$ is the perfusion conductivity, $k_{p} \nabla^{2} T_{t}$ is the conductive heat transfer "enhancement" from the blood flow, $V_{j}^{m}\left(\rho c_{p}\right)_{b}\left(T_{a}-T_{t}\right)$ is the blood perfusion term at the $\mathrm{j}$-th branching blood vessel, and $\left(\rho c_{p}\right)_{b} \vec{v} \cdot \nabla T_{t}$ is the convective transport term, which is directional. The conduction "enhancement" term depends on the blood velocity and the angle between $\nabla T_{t}$ (tissue temperature gradient) and the blood flow [27].

The Chen-Holmes model considers more factors than Pennes' BHTE. The model includes a term for the convection in a thermally significant vessel; it also includes a term that estimates the effects of smaller vessels that are close in temperature to the surrounding tissue (the conduction "enhancement" effect). The convection transport term (in equilibrated vessels) is assumed to be negligible [27]. 
Chen and Holmes quantified the size of what they considered a thermallysignificant vessel in tissue experiencing local heating. They defined a parameter called the thermal equilibration length, $l_{e}$, which depends on the vessel diameter and blood flow parameters. The definition of thermal equilibrium length is,

$$
l_{e}=\frac{A(\rho c)_{b} \bar{V}}{U \cdot P}
$$

where $\bar{V}$ is the average local blood velocity and $U$ is the overall heat transfer coefficient. Using this equation, they found that thermal equilibration of the blood with the surrounding tissue occurs in vessels with diameters from $0.2 \mathrm{~mm}$ to $0.5 \mathrm{~mm}$ [27].

\subsubsection{Varying Vessel Convection Model}

A study conducted by dos Santos et al. [28] considered a single large blood vessel located in a homogeneous block of tissue. They employed a variable convective heat transfer coefficient, $h$, that is a function of the vessel wall temperature along the length of the vessel. The heat transfer equation for the tissue region is,

$$
(\rho c)_{t} \frac{\partial T_{t}}{\partial t}=\nabla k_{t} \nabla T_{t}+\vec{J} \cdot \vec{E}-\rho_{b} \omega_{b} c_{b}\left(T-T_{b}\right)+Q_{m}
$$

Equation 23

where $\vec{J} \cdot \vec{E}$ represents the energy from the radiofrequency ablation device they are modeling. For reasons explained previously, the metabolic heat generation 
term $\left(Q_{m}\right)$ is negligible. The convection at the vessel wall is determined by a convective boundary condition, based on the convection heat transfer coefficient,

$$
h=\frac{\rho c u\left(T_{1}-T_{0}\right) b}{\left[0.25\left(2 T_{s}+T_{1}+T_{0}\right)-T_{m}\right] a}
$$

where $\rho$ and $c$ are properties of blood, $T_{0}$ is the vessel inlet temperature, $u$ is the average laminar blood velocity, $T_{1}$ is the outlet temperature, $T_{S}$ is the maximum temperature at the vessel wall, $T_{m}$ is the mean blood temperature, $a$ is the length of the heated vessel region and $b$ is the vessel diameter.

A related study [29] gives a slightly different approximation for the mean convective heat transfer coefficient at a vessel wall, calculated as,

$$
\bar{h}=\frac{\bar{u}\left(T_{1}-T_{0}\right) b}{\left(T_{s}-T_{m}\right) a}
$$

Models have been proposed and validated for both bulk perfusion approximations and discrete blood vessel convection. For the purposes of predicting HIFU tissue heating, the most appropriate model can be selected based on the quantity and sizes of the blood vessels being modeled, as well as the size of the region being heated. If thermally significant vessels are being modeled and spatially accurate heating predictions are required (as opposed to large-scale bulk heating with less accuracy desired), one of the discrete blood convection approaches should be used. 
The tissue models discussed so far provide many options for predicting heat transfer in perfused tissue, but the predictions can only be as accurate as the HIFU heating source model itself. It is essential that the energy deposited by the HIFU source be modeled accurately, otherwise the heat transfer simulations will not be representative of actual HIFU ablation. Some factors to consider in properly modeling HIFU heating are the absorption and reflection properties of the media (which may be non-linear and/or temperature-dependent), the assumptions made to simplify the wave propagation model, and the method used to solve the wave equations.

\subsubsection{Modeling Ultrasound Heating in Media}

The most accurate way to model the heating characteristics of a HIFU device is to incorporate the ultrasound pressure waves and attenuation into the simulation. Hassan et al. [30] created a numerical simulation of the propagation and interaction of ultrasound through a "multi-tissue type system..." The energy absorption and corresponding temperature increases in the tissue system were calculated analytically and compared to the computer simulations. The study investigated the performance of a circular HIFU transducer at operating frequencies of $0.75 \mathrm{MHz}, 1.5 \mathrm{MHz}$ and $2.75 \mathrm{MHz}$. The study calculated the ultrasound intensity field as, 


$$
I(\varnothing, r, t)=\frac{\pi^{2} a^{4} u_{0}^{2} Z \sin ^{2}(\omega t-k r)}{\lambda^{2} r^{2}}\left[\frac{2 J(k a \sin \phi)}{k a \sin \phi}\right]^{2} \quad \text { Equation } 26
$$

where $a$ is the transducer's radius, $u_{0}$ is the transducer excitation, $Z$ is the acoustic impedance of the medium, $k$ is the ultrasonic wave propagation constant $(2 \pi / \lambda), \mathrm{r}$ is the distance from the source $\left(\sqrt{x^{2}+z^{2}}\right), z$ is the depth and $x$ is the location in the "plane of observation".

Equation 26 can be used to calculate the intensity field in the region ranging from the transducer face to a depth where the intensities have little effect on heating in the medium. This method produces a 3-D HIFU intensity field that may be useful for certain models, but the downside is a relatively large computation time and memory requirement.

Okita et al. [23] describe a developed HIFU simulator that uses real $\mathrm{CT} / \mathrm{MRI}$ images with simulations of ultrasound propagation and energy deposition. Their system uses CT/MRI images to create a model of the patient anatomy. They also created a computer model of a HIFU transducer, which combined with the propagation equations will simulate the multi-source ultrasound propagation and tissue interaction. Figure 5 illustrates the components and steps used by their HIFU simulator. 


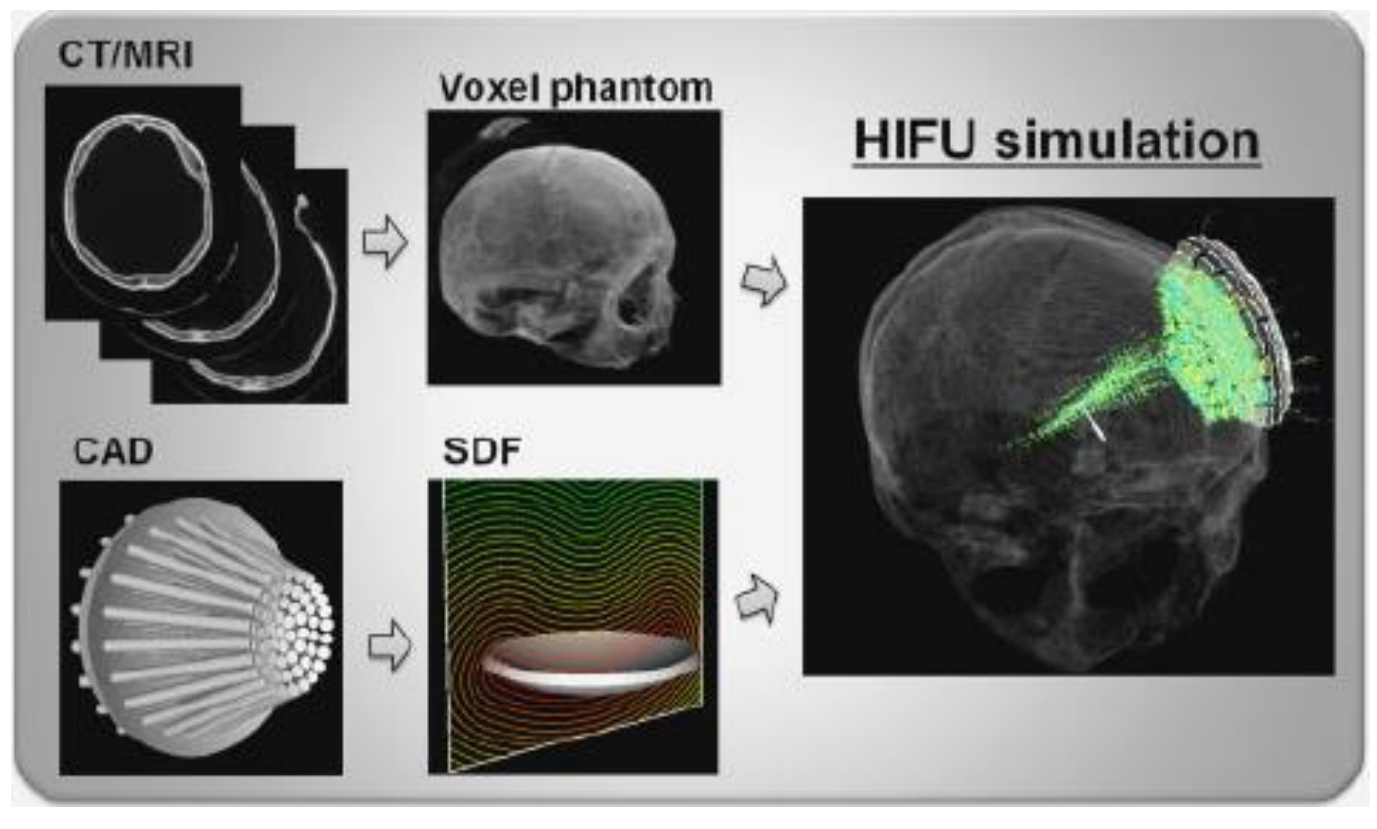

Figure 5. Use of CT/MRI images for HIFU model, reprinted with permission from Okita et al. [23]

\subsubsection{Numerical Simulations of HIFU Heating}

A MATLAB program [31], called 'HIFU_Simulator.m', has been written and published by the FDA to "[determine] many important characteristics of continuous wave, high-intensity focused ultrasound (HIFU) beams and their heating effects." The program utilizes the axisymmetric version of the KZK equation, which is a wave equation that includes effects from diffraction, absorption, and nonlinearity [32]. The simulator finds the pressure field created by a transducer and calculates the intensity and heating rate that results from the sonication. 
This simulation approach is two-dimensional and assumes spherical wave propagation. An example of the program's output, in this case the heating rate and temperature profile of a $3 \mathrm{~cm}$ diameter transducer powered at $100 \mathrm{~W}$ through tissue, is shown below in Figure 6.

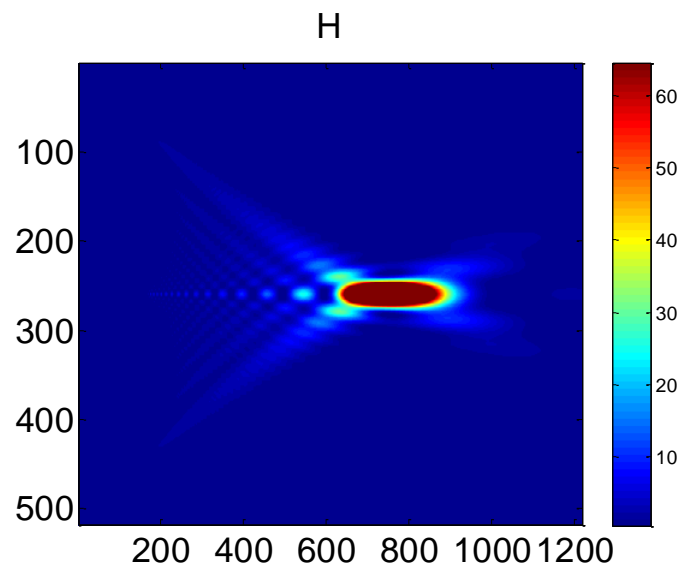

(a)

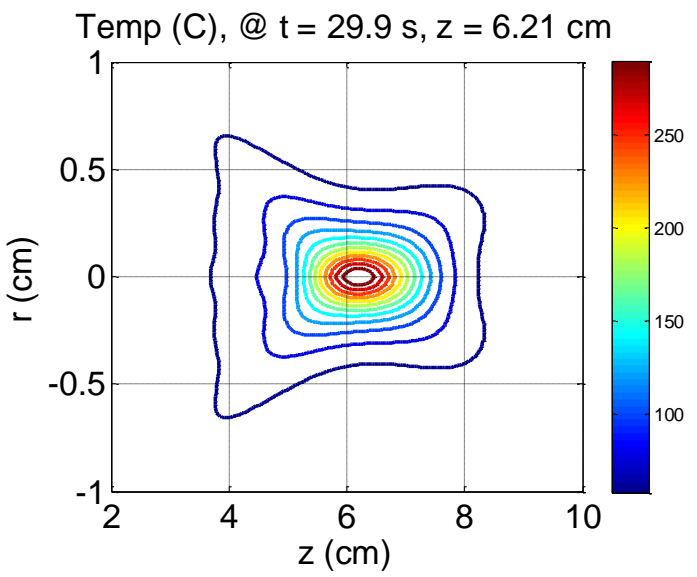

(b)

Figure 6. Plots of simulated (a) heating rate and (b) temperature profile in tissue

For the purposes of this research, the ability to change the model geometry and location of a convective heat sink in the form of a fluid vessel was essential. The models discussed in this section demonstrate the current capabilities in simulating HIFU ablation of tissue. Realistic modeling of most HIFU ablation situations can be accomplished by properly modeling the properties of the ultrasound source and the tissue interaction characteristics. The other method used to investigate HIFU ablation is to use tissue-mimicking materials (TMMs) to create tissue phantoms. Physical phantom models allow for 
real testing of HIFU devices and provide a safe, inexpensive pathway to validate computer models.

\subsection{Tissue-Mimicking Materials (TMMs) for HIFU Ablation}

There are many dangers and difficulties involved in conducting animal and human studies, so alternative methods are useful for developing, testing and evaluating HIFU ablation devices. TMMs, often called "tissue phantoms," have been designed for HIFU applications specifically and present a useful tool for investigating the effects of compression on HIFU ablation. Many different types of TMM have been investigated since HIFU has gained attention as a therapeutic tool. Phantoms based on agar, gelatin, condensed milk, gellan gum and polyacrylamide hydrogel mixed with bovine serum albumin (BSA) have been produced [33]. For the purposes of testing HIFU devices that aim to raise temperatures above $55^{\circ} \mathrm{C}$, the gellan gum TMM and polyacrylamide/BSA TMM are the most tissue-like and robust.

When choosing and building a tissue phantom, the acoustic, thermal, and mechanical properties must be considered. The relevant acoustic and thermal properties generally include the attenuation coefficient, speed of sound through the medium, acoustic impedance, thermal conductivity and thermal diffusivity [33]. For investigating compressive effects, the material stiffness and elastic properties are important as well. As stated by King et al [33], the "HIFU 
phantom should have acoustic propagation characteristics similar to human tissues, both for the linear range as well as the high intensity non-linear regime of ultrasound sonication."

The TMM described by King et al. [33] consists of gellan gum (CG-LA, CP Kelco) mixed with aluminum oxide particles, isopropanol, calcium chloride $(\mathrm{CaCl})$ and potassium sorbate. The elasticity and stiffness of the TMM is largely based on the concentration and type of gellan gum used $(\mathrm{CaCl}$ also affects gel strength). Gellan gum is a non-toxic polysaccharide gelling agent that results in a hydrogel that melts at temperatures above $100{ }^{\circ} \mathrm{C}$. For HIFU applications that require temperatures capable of causing cell necrosis, it is reasonable to assume that temperatures of $80{ }^{\circ} \mathrm{C}$ or $90{ }^{\circ} \mathrm{C}$ will be reached during testing. It is essential that the tissue phantom does not melt and/or change properties drastically during sonication. The aluminum oxide concentration and size range can be changed to alter the absorption/scattering characteristics, while the isopropanol is used to adjust the speed of sound through the TMM [33]. This gellan gum phantom is superior for test repeatability, user safety and thermal response to HIFU, but it is opaque and thermal lesions cannot be visualized.

Polyacrylamide/BVA TMM is a transparent material that is being used for HIFU phantoms. The BVA component becomes opaque after it passes its critical temperature (generally around $70{ }^{\circ} \mathrm{C}$ ) due to protein denaturation [34]. Figure 7 
shows two separate HIFU lesions made in a polyacrylamide/BVA gel made by Onda Corporation (Sunnyvale, California, USA).

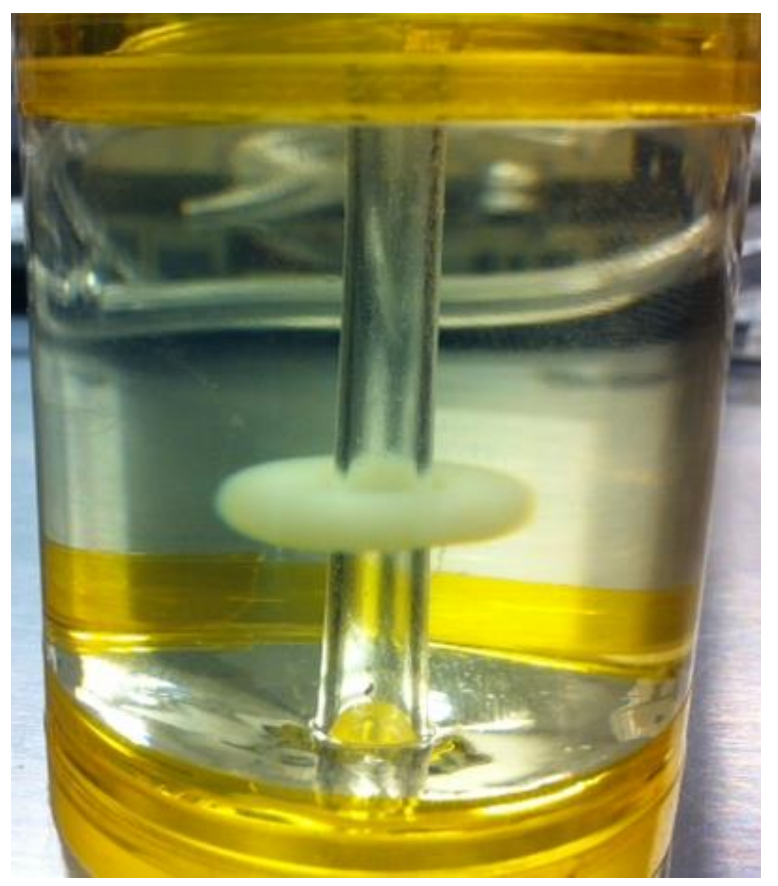

Figure 7. Side-view of a polyacrylamide/BVA vessel phantom with a circumferential lesion made by ultrasound (property of ReCor Medical)

The TMM described by Lafon et al. [34] is based on a polyacrylamide/BVA mixture with adjustable attenuation properties that can be used to mimic different tissues. The authors state that this phantom "would facilitate dosimetry studies, transducer evaluation, and the development of theoretical and numerical models of HIFU treatments." The ability to visualize and, consequently, measure the shape and size of the created thermal lesion will allow for direct comparison to computer simulations. This can provide validation of a computer model, which would open the door for more complicated simulations of perfused tissue 
models. In addition, it can be used as a stand-alone indicator of HIFU ablation performance.

2.4 Research on the Effects of Blood Vessels on HIFU Ablation

Multiple published studies have shown that blood perfusion can have a significant effect on HIFU ablation effectiveness and predictability. Huang et al. [35] recommend decreasing or preventing blood flow rates in tumorous regions before treatment. Dasgupta et al. [17] built a thermal HIFU phantom with a vessel running through it in order to investigate the effect of flow rate on local temperature distribution. The study found more than a $10 \%$ decrease in peak temperature in the region close to the vessel and HIFU focus. Their experimental setup is shown in Figure 8.

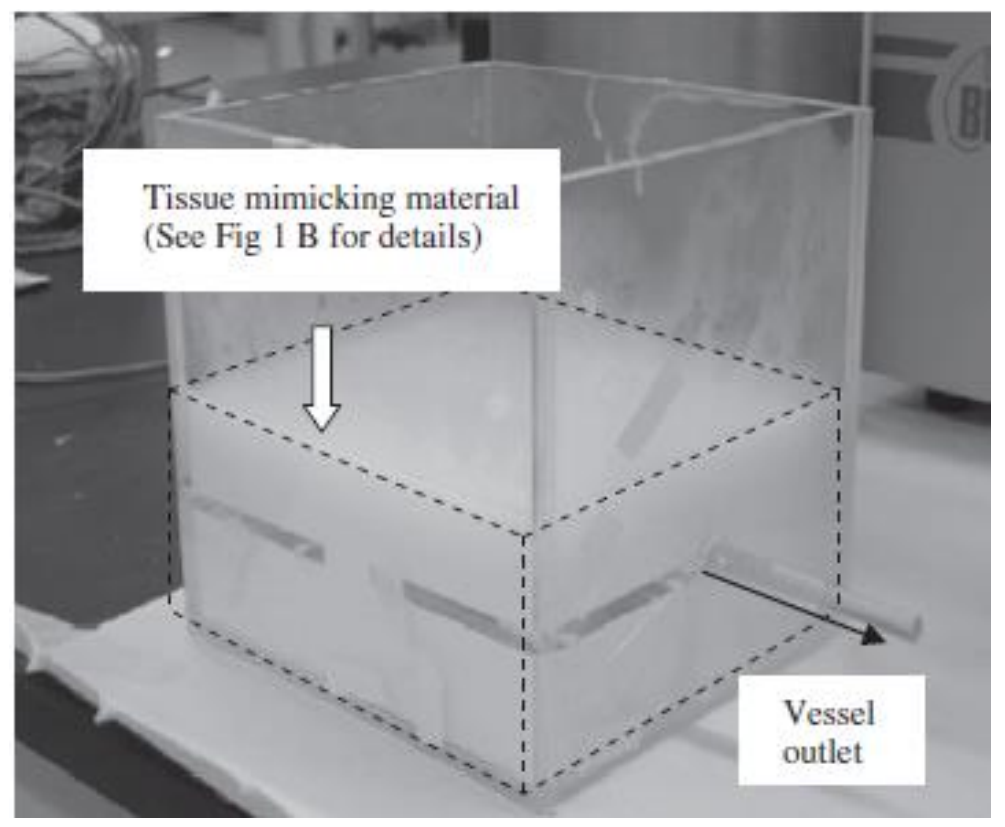




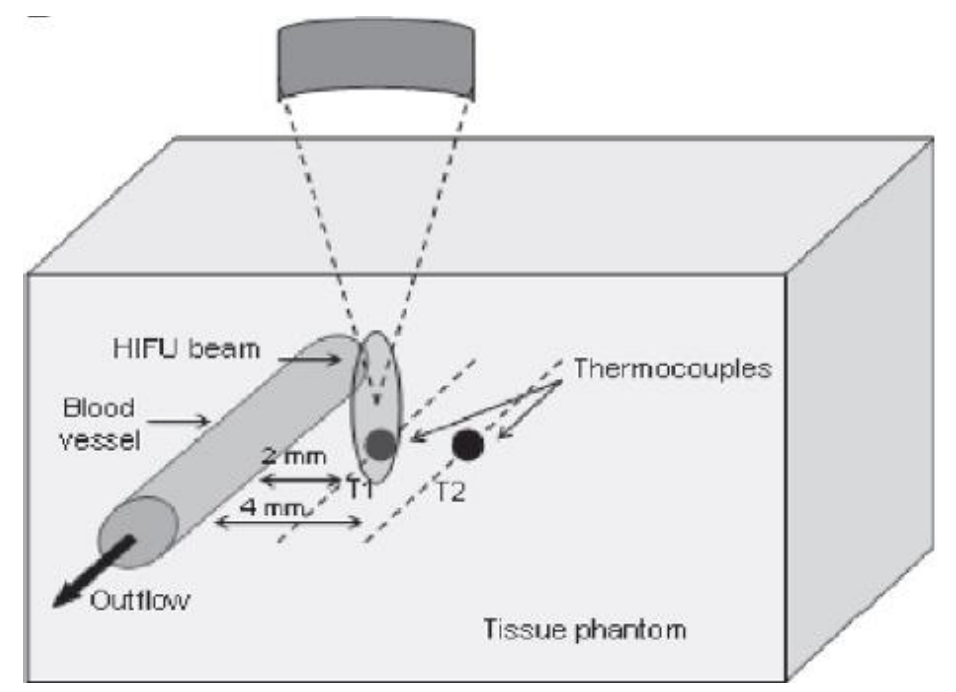

Figure 8. HIFU phantom with vessel and embedded thermocouples, reprinted with permission from Dasgupta et al. [17]

Using finite-element analysis (numerical technique), Jarosz [36] found that when blood vessels with diameter between $0.4 \mathrm{~mm}$ and $1.1 \mathrm{~mm}$ are on the perimeter of a HIFU-targeted region, either higher ultrasonic powers or durations are required to complete treatment. This would result in "heat toxicity" in the healthy surrounding tissues. He also found that with largediameter vessels in the targeted region, certain locations will not reach the predicted therapeutic thermal dose. Large vessels on the perimeter of the treatment zone can also keep the region from reaching therapeutic temperatures.

A study done by Sassaroli et al. [37] investigated the impact of blood vessel flow on tissue temperature profile during HIFU ablation. The simulation, done with COMSOL Multiphysics (COMSOL, Inc., Burlington, Massachusetts, USA), uses a "multiple artery-vein system located in a homogeneous block of 
muscle-like tissue..." The model simulates the power density (axial and radial) of the HIFU transducer, and then applies that power source to the tissue/vessel geometry shown in Figure 9.

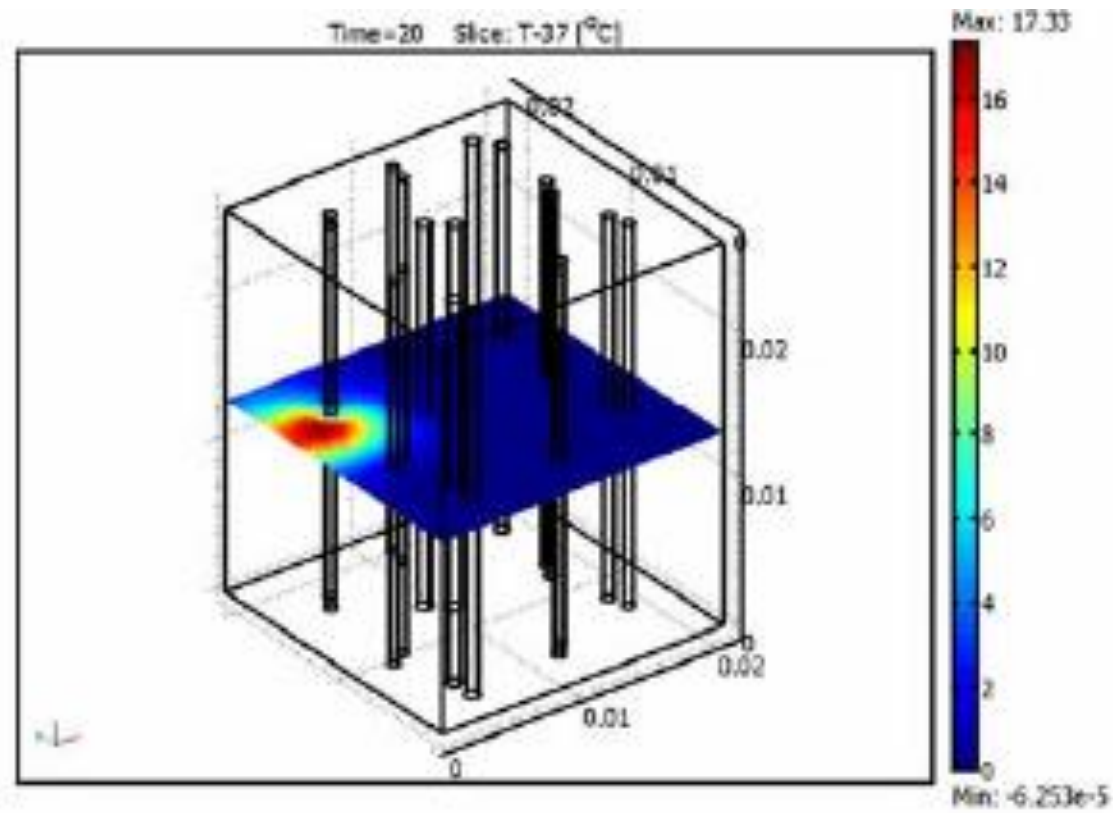

(a)

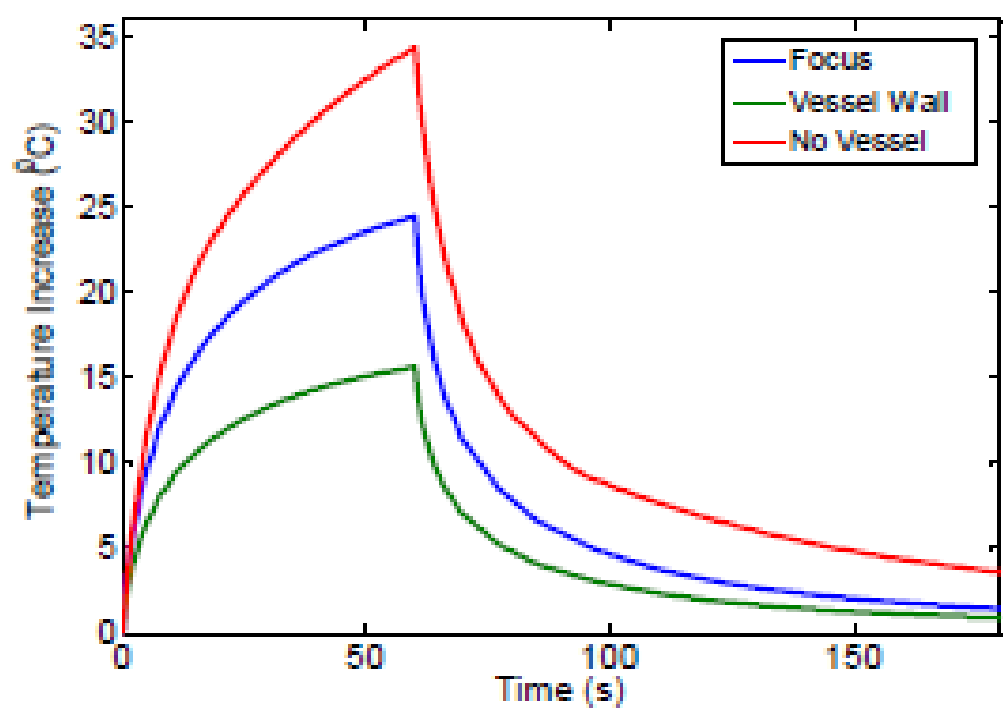

(b)

Figure 9. (a) Tissue/vessel geometry and (b) temperatures with and without vessels, reprinted from COMSOL open-source website [37] 
The tissue block was sonicated at the mid-plane, both with random positions ("to avoid thermal build-up") and in a spiral path. The "thermal dose" was then calculated and compared to a reference dose that corresponds with tissue necrosis:

$$
T D(t)=\int_{0}^{t} \mathcal{R}^{43-T\left(t^{\prime}\right)} d t^{\prime}
$$

with

$$
\mathcal{R}=\left\{\begin{array}{cc}
0.25 & T(t)<43^{\circ} \mathrm{C} \\
0.5 & T(t) \geq 43^{\circ} \mathrm{C}
\end{array}\right.
$$

The simulations suggested that there was insufficient thermal dosage near the blood vessels, which can be seen in the cold spots (dark blue in plot) in Figure 10.

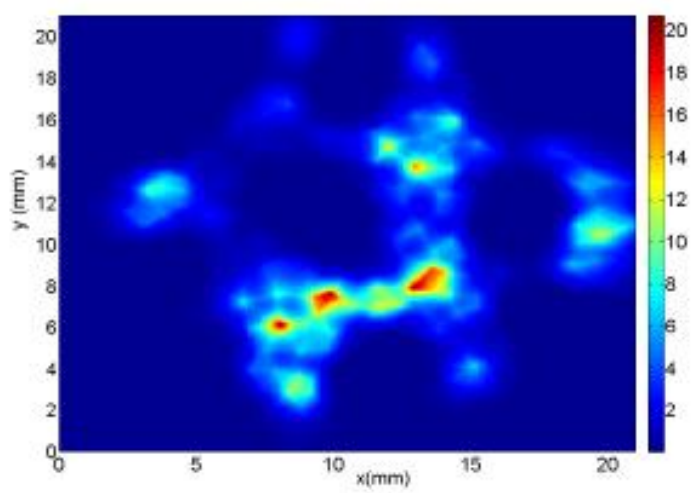

(a)

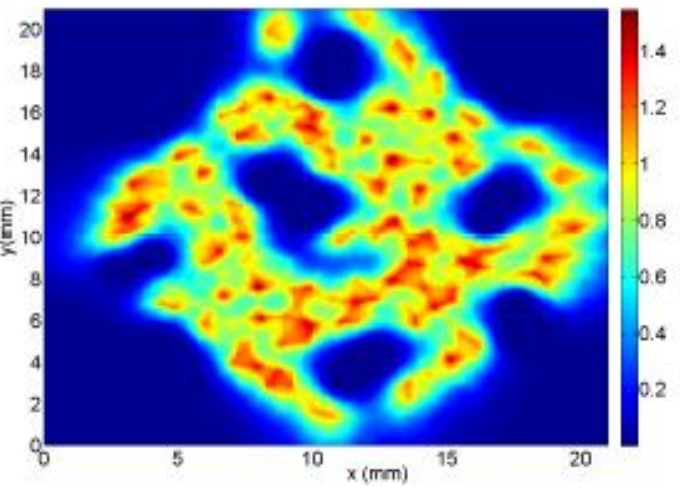

(b)

Figure 10. (a) Thermal dose mid-plane with fixed power and (b) with variable power, reprinted from COMSOL open-source website. [37] 
The authors state that "blood flow strongly affects the temperature distribution during an ultrasound thermal treatment." According to this study, if the heat sink effect of the blood vessels is not taken into consideration, there will be high variability in the actual thermal dose. The authors recommend that imaging be done to determine the location and size of "thermally significant" blood vessels (diameter between $0.3 \mathrm{~mm}$ and $0.8 \mathrm{~mm}$ ) in each patient [37]. A review of the literature clearly shows that blood flow near a HIFUtargeted area significantly affects the heating characteristics. HIFU heating has been investigated experimentally, as well as through computer simulations. The KZK equation is a powerful tool in approximating nonlinear ultrasound propagation, allowing researchers to simulate a variety of physical situations. Tissue phantoms have proven to be useful tools as well, both as stand-alone evidence and as validation of simulation techniques. This thesis approaches the problem of HIFU ablation in perfused tissue using both phantoms and simulations. 


\section{CHAPTER THREE HYPOTHESIS AND OBJECTIVES}

Much work has been published on the development and validation of models of tissue heat transfer and ultrasound interaction [23, 30, 31]. Studies have shown that it is possible to simulate ultrasonic wave propagation from HIFU transducers, as well as the interaction that occurs as it passes through a medium such as soft tissue. Computer simulations of HIFU ablation and other thermal therapies have been created by many researchers. In addition to computer simulations, materials have been developed that can accurately mimic HIFU tissue heating. Using polyacrylamide/BSA and the gellan gum phantoms, researchers can measure the temperature profiles during sonication and observe the ablation region through changes in opaqueness.

Through a combination of computer simulations and tissue phantom experiments, it has been shown that blood vessels have a significant effect on HIFU ablation. "Thermally significant" vessels can make it difficult to model and predict HIFU treatment. Treatment planning often relies on experimental or simulated data of HIFU heating, but the presence of complex vasculature complicates the process. Unless clinicians were to run heating simulations with full vessel architecture and blood flow directionality included, predications of HIFU heating in the perfused target region would be inaccurate. Sonication 
power and/or duration can be increased to compensate for the heat being lost to convection through the blood vessels, but at the cost of increased risk of tissue damage in the surrounding tissue regions.

A clear relationship between vessel flow rate and peak temperatures near the vessel during HIFU ablation was shown by Dasgupta et al. [17], but this study altered the flow rate non-locally (either with pumps or a reservoir system). There is no known research that investigates the effects compression has on local vessels and HIFU heating. This approach has the potential to give patients and clinicians confidence that they are safely and effectively treating cancer, without the worry that unpredictable convection is occurring and altering the planned treatment.

\subsection{Hypothesis}

Eliminating factors that complicate the modeling of HIFU heat transfer can make treatment planning easier and more accurate. The hypothesis of this investigation is that tissue compression can improve spatial heating uniformity by decreasing blood flow rate in vessels near a HIFU target.

\subsection{Research Objectives}

To investigate the hypothesis, the approach is to use compression to reduce or cut off fluid flow near a HIFU target prior to ablation. If flow can 
effectively be reduced or eliminated within $1 \mathrm{~cm}$ of the target perimeter, research suggests that HIFU treatment will be more uniform and easier to predict [17]. A combination of computer simulations and tissue phantom studies will be used to study the effect of compression on: (a) blood flow through vessels near a target (i.e., tumor) region, and (b) the heating profile during HIFU ablation. The specific objectives of the studies are:

* Develop numerical tools to simulate HIFU intensity and tissue temperature profiles, including convection at the boundary of a fluid vessel.

* Predict temperature distribution as a function of flow rate through an embedded fluid vessel.

* Measure the flow rate through a vessel embedded in tissue-mimicking material, as a function of compression.

* Develop a tissue phantom and apparatus to measure the temperature distribution as a function of compression during HIFU heating. The next chapter discusses the experimental methods and materials and includes justifications for all assumptions made. 


\section{CHAPTER FOUR \\ METHODOLOGY}

The hypothesis of this research was investigated using an experimental approach combined with a computer simulation model. The two approaches were set up to be directly comparable with the goal of validating the numerical model with the experimental results.

\subsection{Model Setup and Numerical Simulation}

A model was designed to match the various geometric and physical properties of the experimental setup during HIFU heating and compression in an effort to compare the experimental and simulated results. The goal was to validate the model with the experimental results, thus providing justification to expand the model's complexity in ways that would be difficult or impossible with a tissue-phantom.

\subsubsection{Physics Modeling and Assumptions}

Actual perfused soft tissues, especially in tumorous regions, are heterogeneous and practically impossible to model on an individual patient basis, so multiple assumptions were made to simplify and generalize modeling. All material properties were assumed to be constant, disregarding any temperature-dependent and compression-dependent properties. Studies show 
that the TMM used in this investigation has temperature-dependent thermal conductivity, speed of sound, attenuation, and nonlinear properties, but the variation was deemed insignificant for the modest temperature extremes of only a few tens of degrees Celsius. It is also likely that compression of the TMM affects the thermal conductivity and acoustical properties, but publications documenting these relationships were not discovered. Compression-dependent properties would be useful to consider in future studies, but have been omitted from the scope of this investigation. Table 2 shows the properties used in the HIFU beam and heat transfer simulations.

Table 2. Properties used in model

\begin{tabular}{|c|c|c|c|}
\hline \multicolumn{2}{|c|}{ Sound speed $(\mathrm{m} / \mathrm{s})$} & \multicolumn{2}{|c|}{ Power of Attenuation vs. Frequency } \\
\hline Cwater & 1482 & $\gamma_{\text {water }}$ & 2 \\
\hline Стмм & 1630 & $\gamma_{\text {TMM }}$ & 1 \\
\hline Cblood & 1570 & $\gamma$ blood & 1 \\
\hline \multicolumn{2}{|c|}{ Density $\left(\mathrm{kg} / \mathrm{m}^{3}\right)$} & \multicolumn{2}{|c|}{ Non-linear Parameter $(1+B / 2 A$} \\
\hline Qwater & 1000 & $\beta_{\text {water }}$ & 3.5 \\
\hline @тмМ & 1030 & $\beta_{\text {TMM }}$ & 8.0 \\
\hline Qblood & 1060 & $\beta$ blood & 4.1 \\
\hline \multicolumn{2}{|c|}{ Attenuation $(\mathrm{dB} / \mathrm{m})$} & \multicolumn{2}{|c|}{ Material transition distances (m) } \\
\hline$\alpha_{\text {water }}$ & 0.217 & Zwater & 0.001 \\
\hline$\alpha_{\text {TMM }}$ & 68 & ZTMM & 0.20 \\
\hline$\alpha$ blood & 1.61 & Zblood & $\mathrm{N} / \mathrm{A}$ \\
\hline
\end{tabular}




\begin{tabular}{|l|l|}
\hline \multicolumn{2}{|c|}{ Parameters Transducer (m) } \\
\hline Radius & 0.035 \\
\hline Focus (d) & 0.10 \\
\hline
\end{tabular}

The initial concept for this investigation was to simulate bioheat transfer during HIFU ablation near a blood vessel, but it became clear that modeling the perfused tissue phantom and HIFU source would be more appropriate. Avoiding the bioheat transfer equation by modeling the simplified TMM medium instead of tissue allows for a direct comparison between the simulations and the experimental results. Assumptions about the blood perfusion rate or "effective conductance" of the tissue were also avoided, making the model setup and numerical solution much simpler.

\subsubsection{HIFU Beam Simulation}

The program used to simulate the HIFU beam intensity profile is an extension of a public software package made available by the U.S. Food and Drug Administration (FDA) [31]. The package consists of 19 MATLAB scripts (code in Appendix A) that discretize the assigned geometry and calculate the ultrasonic beam properties based on the KZK equation. The simulation can output the beam's pressure, intensity and heating rate profiles in matrix form. 
The simulation assumes a round, concave transducer of radius $r$ that produces a focused beam with focus depth $d$ (see Table 2). A $0.25 \mathrm{~cm}$ thick perfectly-matched layer (PML) at the surface of the transducer is also assumed to be part of the model. The computational grid is built by discretization of the full computational domain. The domain size is equal to the transducer radius in the radial direction and twice the focal depth in the axial direction. The grid is formed by breaking up the domain into axial and radial step-sizes, displayed as $d z$ and $d r$ respectively. The step sizes for this simulation are based on the linear pressure gain and the estimated points per wavelength in each direction. The simulation designates 20 points per wavelength in the axial direction and 30 points in the radial direction. These values represent "a good balance between solution accuracy and computational efficiency..." [31] The discretization parameters of the beam simulation are displayed by the software as,

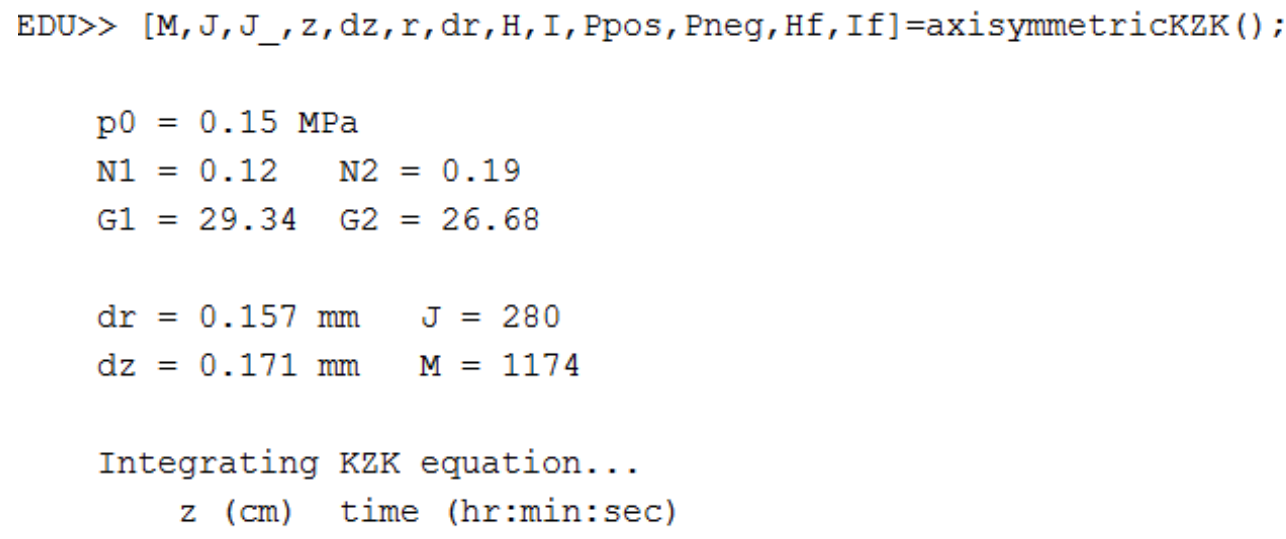

The beam simulation was run using the material properties of the gellangum based TMM and the transducer properties (geometry, frequency and 
power) from the Sonic Concepts (Sonic Concepts, Inc., Bothell, Washington,

USA) H-101 transducer used in the experimental portion of this investigation.

The resulting intensity and heating rate plots are shown in Figure 11.

(a)

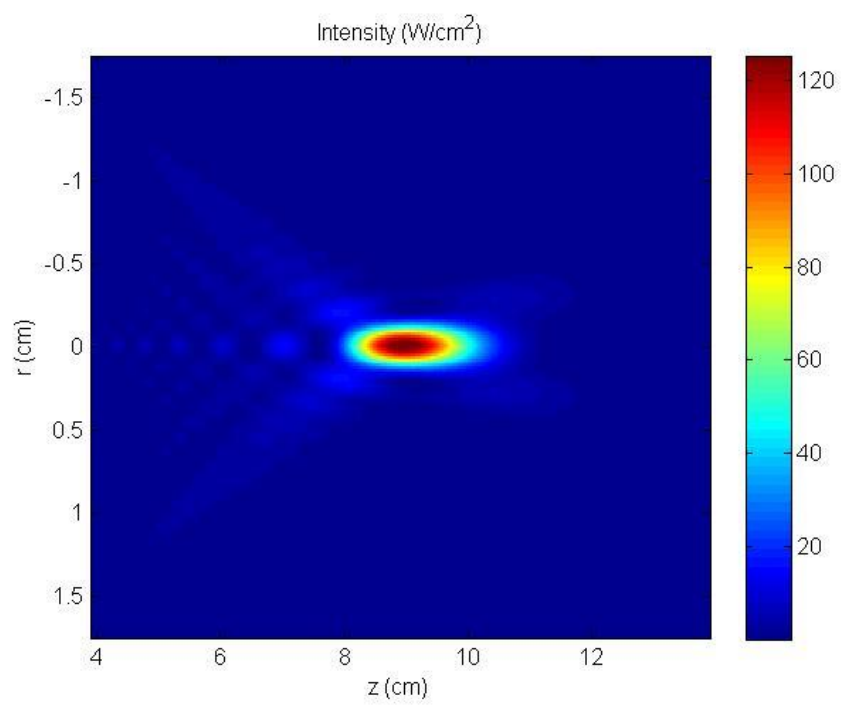

(b)

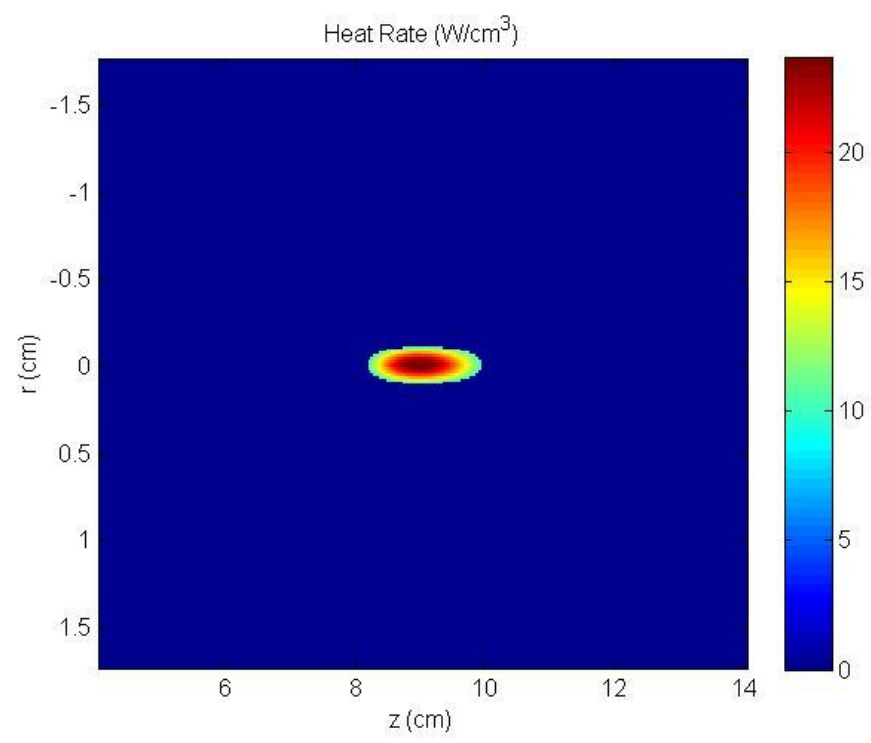

Figure 11. (a) Intensity and (b) heating rate of $1.13 \mathrm{MHz}$ HIFU transducer at $30 \mathrm{~W}$ 
The heating rate can subsequently be used as the heat generation term in the heat equation. For direct compatibility between the two models, the same grid domain size and node spacing was used in developing the finite-difference equations for solving the heat equation.

\subsubsection{Numerical Simulation of Heat Transfer}

The two-dimensional heat transfer simulation is solved using the explicit finite-difference (FD) scheme (see Section 1.2.4). The computational grid is $7 \mathrm{~cm}$ in the radial direction and $20 \mathrm{~cm}$ in the axial direction, with radial and axial stepsizes taken directly from the HIFU beam simulation. The equation that is solved includes a conduction term for heat transfer through the TMM, a convection term for heat transfer at the vessel-TMM interface and a heat generation term from the HIFU source. The heat generation is governed by the output of the HIFU beam simulation. The equations used in the explicit FD scheme are derived via the finite-volume energy balance method. Each node in the two-dimensional grid domain is assumed to have a finite volume associated with it. An example of a node at location $(m, n)$ is shown in Figure 12. 

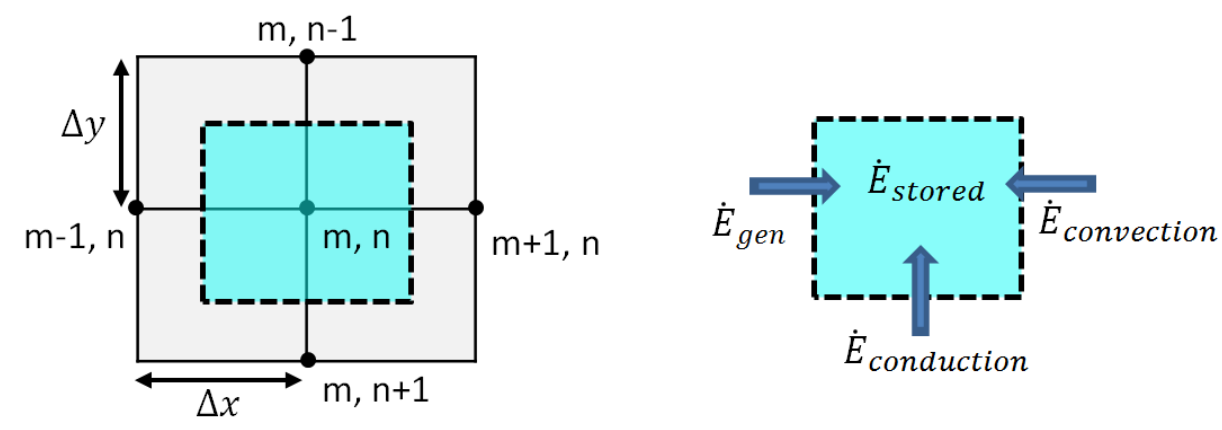

Figure 12. Example of interior node and energy balance

Energy balance within this finite region dictates that the energy generated by the HIFU source plus the energy transferred into the region by conduction must be equal to the energy lost through convection. The energy balance method leads to the explicit FD equations for every node in the model. The nodes can be split into 3 basic types:

1) Perimeter/boundary nodes

2) Interior nodes with no convection

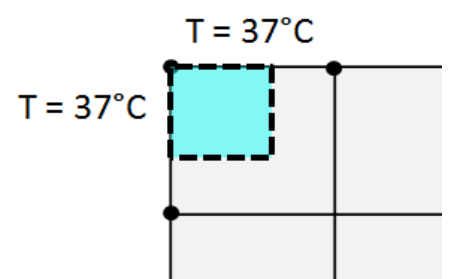

1)

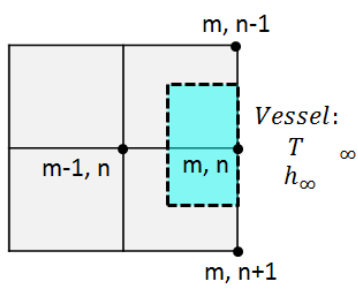

3)

3) Interior nodes with convection

The other factor in the energy balance, the heat generated by the HIFU source, is assumed to be present in every node. This assumption is made because the heat generation term is imported directly from the HIFU beam 
simulation in the form of a matrix equal in size to the entire model grid. At most nodes, the heat generation is zero, but it is still included in all the node equations.

The FD equations were developed for each of the three node types shown above, and solved with commercial computations software. The conditions at the perimeter boundaries of the model were set to a constant temperature, approximately equal to room temperature $\left(23^{\circ} \mathrm{C}\right)$. The next node type, which involves conduction and heat generation without convection, has a FD equation of the form,

$$
\begin{gathered}
\left(T^{t+1}{ }_{i, j}-T^{t}{ }_{i, j}\right)=\propto \\
\Delta t\left[\frac{T^{t}{ }_{i-1, j}-2 T^{t}{ }_{i, j}+T^{t}{ }_{i+1, j}}{(\Delta x)^{2}}+\frac{T^{t}{ }_{i, j-1}-2 T^{t}{ }_{i, j}+T^{t}{ }_{i, j+1}}{(\Delta y)^{2}}\right]+\frac{\Delta t}{\rho c_{p}} q_{i, j}
\end{gathered}
$$

Equation 28

where $\propto=k /\left(\rho c_{p}\right)$ and $q_{i, j}$ is the HIFU heating source. The nodes along the boundary of the vessel have an additional convection term, resulting in a FD equation of the form,

$$
\begin{gathered}
\left(T^{t+1}{ }_{i, j}-T^{t}{ }_{i, j}\right)=\propto \Delta t\left[\frac{{T^{t}}_{i-1, j}-2 T^{t}{ }_{i, j}+T^{t}{ }_{i+1, j}}{(\Delta x)^{2}}+\right. \\
\left.\frac{T^{t}{ }_{i, j-1}-2 T^{t} t_{i, j}+T^{t}{ }_{i, j+1}}{(\Delta y)^{2}}\right]+\frac{h \Delta t}{\rho c_{p} \Delta x}\left(T_{\infty}-T^{t}{ }_{i, j}\right)+\frac{\Delta t}{\rho c_{p}} q_{i, j}
\end{gathered}
$$

where $T_{\infty}$, is the temperature of the fluid flowing through the internal vessel, which is assumed to be constant at $23^{\circ} \mathrm{C}$. Since the problem being solved in this 
situation is two-dimensional, the convection term in Equation 29 may involve $\Delta y$ instead of $\Delta x$, depending on the direction of convective heat flow (either in axial or radial direction). The convective heat transfer coefficient is calculated as,

$$
\bar{h}=\rho_{f} c_{p, f} \frac{\bar{u}\left(T_{f, 1}-T_{f, 0}\right) d}{\left(T_{s}-T_{m}\right) a}
$$

Equation 30

where $d$ is the diameter of the vessel, $\bar{u}$ is the mean fluid velocity, $T_{f, 0}$ is the temperature of the fluid at the inlet, $T_{f, 1}$ is the temperature at the outlet, $T_{S}$ is the temperature of the heated surface of the vessel, $T_{m}$ is the mean fluid temperature and $a$ is the heated length of the vessel. The difference between the inlet and outlet temperatures of the vessel is assumed to be $0.05^{\circ} \mathrm{C}$. $T_{S}$ is assumed to be 50 ${ }^{\circ} \mathrm{C}$ based on the temperature limit for tissue necrosis [36]. The other thermal properties used in the simulation are shown in Table 3.

Table 3. Thermal properties for heat transfer simulation

\begin{tabular}{|c|c|c|c|}
\hline \multicolumn{2}{|c|}{ Thermal Conductivity (W/m·K) } & \multicolumn{2}{c|}{ Heat Capacity (J/kg.K) } \\
\hline $\mathrm{k}_{\text {vessel }}$ & 0.51 & Cwater & 4190 \\
\hline $\mathrm{k}_{\text {TMM }}$ & 0.6 & CTMM & 4180 \\
\hline Vessel/Fluid Parameters & $T_{S}$ & $50^{\circ} \mathrm{C}$ \\
\hline$T_{\infty}$ & $23^{\circ} \mathrm{C}$ & $T_{m}$ & $37^{\circ} \mathrm{C}$ \\
\hline$T_{f, 0}$ & $37^{\circ} \mathrm{C}$ & a & $2 \mathrm{~cm}$ \\
\hline$T_{f, 1}$ & $37.05^{\circ} \mathrm{C}$ & & \\
\hline
\end{tabular}


In order to investigate the effect of compression on the simulated heating profile, progressively more compression was assumed in the model along with corresponding changes in the flow rate and vessel geometry. The flow rates used in the simulations were chosen based on the experimental results in order to give a representative collection of temperature profiles to compare.

\subsection{Experimental Methods}

The effect of compression on vessel flow rate and the heating profile inside a perfused tissue phantom were determined through an experimental approach, which was then compared with the computational approach. The experimental approach required a HIFU source and transducer positioning system, a thermal phantom (melting point $>100{ }^{\circ} \mathrm{C}$ ) with a vertical vessel, a reservoir system with adjustable flow settings, a means of measuring the flow rate through the vessel and a fixture designed to record transient temperatures throughout the phantom during sonication. A diagram summarizing the setup is shown in Figure 13. 


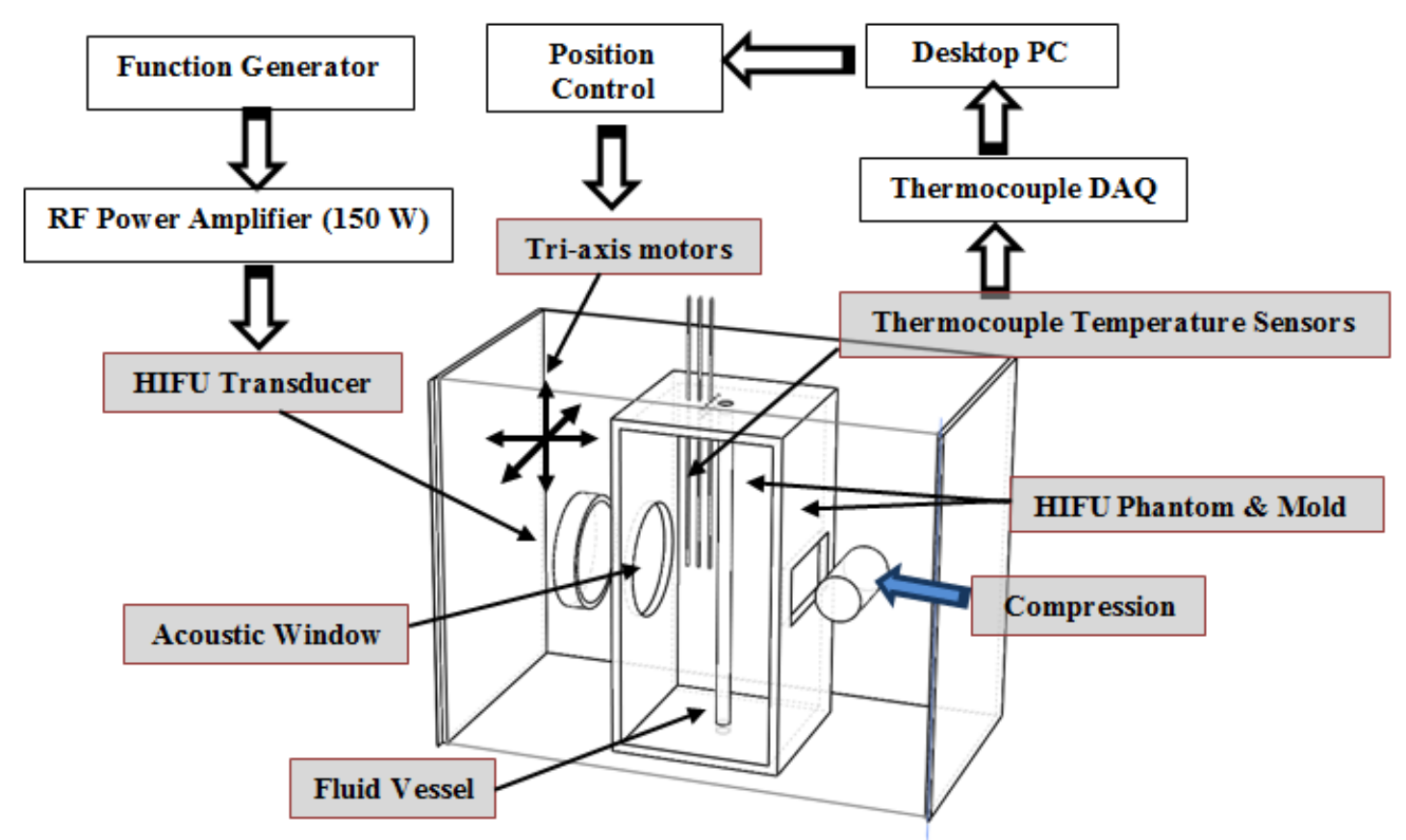

Figure 13. Experimental setup showing the phantom and HIFU fixtures

\subsubsection{Tissue Phantom Materials and Preparation}

The HIFU tissue phantom used in this investigation is a gellan gum hydrogel matrix (see Section 2.3). Gellan gum is non-toxic and produces a hydrogel with “higher temperature stability (melting point $>100{ }^{\circ} \mathrm{C}$ ), higher mechanical strength, and better clarity than traditional agar or gelatin based media." [11] The exact materials used to create the phantom were:

- Gellan gum (CG-LA, CP Kelco)

- Aluminum oxide particles (various sizes between 0.1-100 $\mu \mathrm{m}$ )

- Isopropanol (1-propanol)

- Calcium chloride $(\mathrm{CaCl})$ 
- Potassium sorbate

- Degassed and deionized water

The steps for making $500 \mathrm{~mL}$ of TMM, as described by King et al., are:

1. Using a vacuum filter flask (Erlenmeyer flask) on a magnetic stir/hot plate, mix $2 \mathrm{~g}$ of calcium chloride and $0.5 \mathrm{~g}$ of potassium sorbate with $100 \mathrm{~mL}$ of degassed water.

2. Cap the flask and attach the outlet nozzle to a vacuum pump. Continue to mix, under a 27 in $\mathrm{Hg}$ vacuum, this solution for the rest of this procedure.

3. In a second filter flask, mix $2.7 \mathrm{~g}$ of aluminum oxide particles (combination of three sizes) and $56.25 \mathrm{~mL}$ of 1-propanol with $400 \mathrm{~mL}$ of degassed water.

4. Cap the flask and attach the outlet nozzle to a second vacuum pump. Mix on a second stir/hot plate under 27 in $\mathrm{Hg}$ vacuum for 1 hour.

5. Add $7.5 \mathrm{~g}$ of gellan gum to the aluminum oxide-isopropanol solution using a paper or plastic tube positioned just above the surface of the solution. Add the gellan gum slowly, while the stir plate continues to mix, taking care to avoid clumping. If the gellan gum clumps, reapply vacuum and allow the solution to mix until the clumps disappear.

6. Turn on the heaters on the stir/hot plates for both solutions. Bring the $\mathrm{CaCl}$ solution to $80{ }^{\circ} \mathrm{C}$ and the gellan gum solution to boiling. 
7. Allow the gellan gum solution to boil for 2-3 minutes to ensure consistency.

8. Turn down the heat (keep stirring) on the gellan gum solution and allow it to cool to $80{ }^{\circ} \mathrm{C}$. Add the $\mathrm{CaCl}$ solution (still at $80{ }^{\circ} \mathrm{C}$ ) and continue stirring the final solution.

9. Cool the final solution to $70{ }^{\circ} \mathrm{C}$ and pour quickly into the phantom mold.

10. Allow the phantom to congeal and cool down for at least two hours.

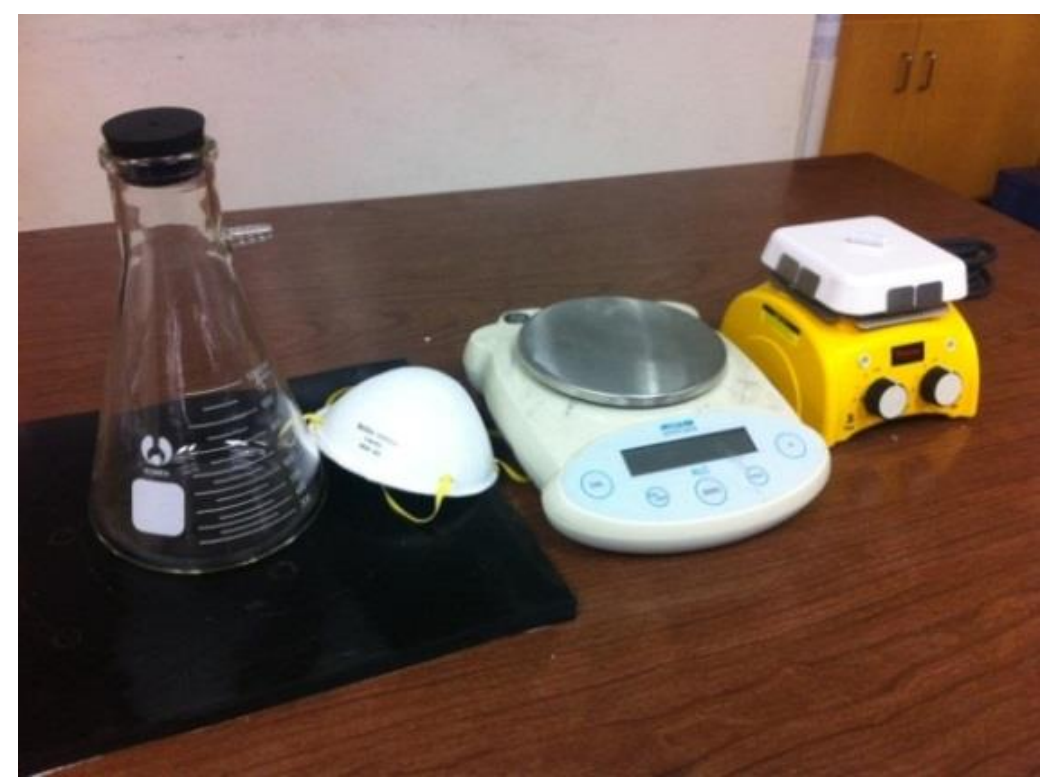

Figure 14. Equipment used for phantom fabrication

Before the TMM was poured into the mold, the mold was placed in boiling water for 1 minute to sterilize the surfaces. An acrylic rod was positioned inside the mold before it was filled to create the wall-less fluid vessel. The rod was inserted through the pre-drilled holes in the mold and secured in place 
using a hot-glue gun. The rod was then sprayed with a non-stick agent that made it easy to remove after the TMM had congealed in the mold. The void created in this process was designed to mimic a blood vessel in size and mechanical characteristics. Figure 15 shows the empty phantom mold with the thermocouple array that remained embedded inside the TMM. The finished product (Figure 15b) features a solid phantom with a single vessel running vertically.

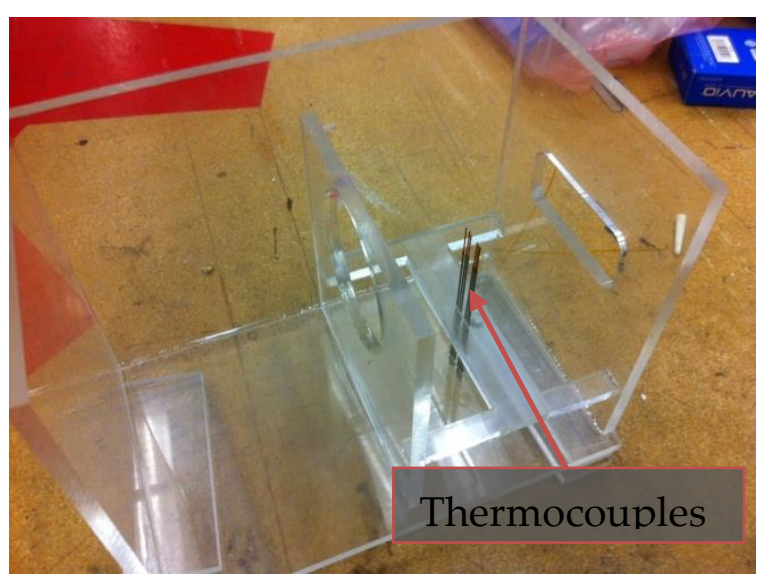

(a)

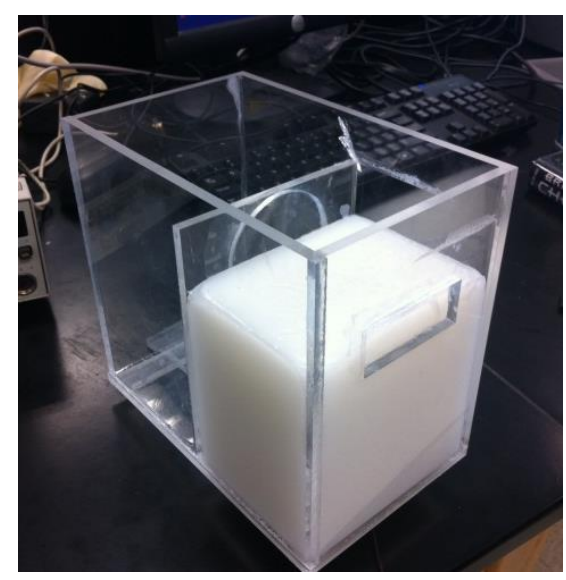

(b)

Figure 15. Phantom mold with thermocouple fixture (a) before TMM is added and (b) after addition of TMM

The phantom mold was designed using SolidWorks CAD software and built partly in the San Jose State University machine shop and partly at Tech Shop (San Jose, CA). All of the mold sections were made from transparent acrylic. The mold includes an ultrasound window that allows HIFU to propagate into the phantom without interference. The window is a circular cut- 
out with a diameter about the same as the HIFU transducer's diameter. On the side opposite the HIFU window, there is another cut-out that allows for compression of the phantom. The compression applicator was built from a halfcylinder rod cut to fit through the compression window of the mold. The bottom of the mold featured a hole sized to accommodate a Luer fitting so that tubing connections could be attached to the phantom's internal vessel. Also on the bottom surface are small holes for the thermocouple probes, which were embedded in the phantom material.

\subsubsection{Experimental Apparatus}

The experimental setup has four main components in addition to the phantom, being the fluid flow system, the HIFU driving and positioning system, the compression system and the temperature sensing system. The systems were built and tested individually final assembly.

The fluid flow system consists of an acrylic fluid reservoir, approximately $90 \mathrm{~cm} \times 90 \mathrm{~cm} \times 10 \mathrm{~cm}$, which supplies a constant flow (which can be adjusted) to the HIFU phantom. The reservoir was placed on an adjustable-height platform that could be moved to change the flow rate. In a reservoir system, flow rate is governed by Bernoulli's principle, which relates fluid velocity with pressure and the potential energy of the fluid. In this reservoir system, the potential energy of 
the fluid is controlled by adjusting the height of the upper reservoir. The basic governing equation for the flow through the system is,

$$
\frac{1}{2} \rho v^{2}+\rho g z+p=\text { constant }, \quad \text { Equation } 31
$$

where $\rho$ is the fluid density, $v$ is the fluid velocity, $g$ is gravitational acceleration, $z$ is the height of the fluid above a specified neutral height and $p$ is pressure. In a real system, Equation 31 does not sufficiently describe the physics, so a modified equation was substituted [38]. Taking into account gravitational acceleration, fluid density and viscous losses through the tubing system, the equation for flow rate is,

$$
Q=\frac{D^{4} g \rho_{0} H}{128 \mu L}
$$

where $D$ is the vessel diameter, $g$ is gravitational acceleration, $\rho_{0}$ is the fluid density, $H$ is the head loss, $\mu$ is the fluid viscosity and $L$ is the total length of the tubing. Equation 32 gives a relationship between the fluid height and the flow rate, allowing for a calculation of the reservoir height that will result in the desired flow rate of the fluid. Normal blood flow through human arteries is generally between $0.1 \mathrm{~cm} / \mathrm{s}$ and $100 \mathrm{~cm} / \mathrm{s}$ [38]. This study started with a flow rate of $40 \mathrm{~mL} / \mathrm{min}$, or approximately $0.6 \mathrm{~cm} / \mathrm{s}$, which is within the range of flow through arteries having caliber between $2 \mathrm{~mm}$ and $6 \mathrm{~mm}$. 
Once the reservoir height was calculated and the reservoir was positioned, tubing was attached to the outlet nozzle on one end of the phantom and to the inlet nozzle on the other end. Outlet tubing was also connected to the nozzle located on the bottom of the phantom. The outlet tubing directed the fluid to a graduated cylinder that was used to measure the flow rate. The experimental setup (magnetic mixer and graduated cylinder not visible) is shown in Figure 16.

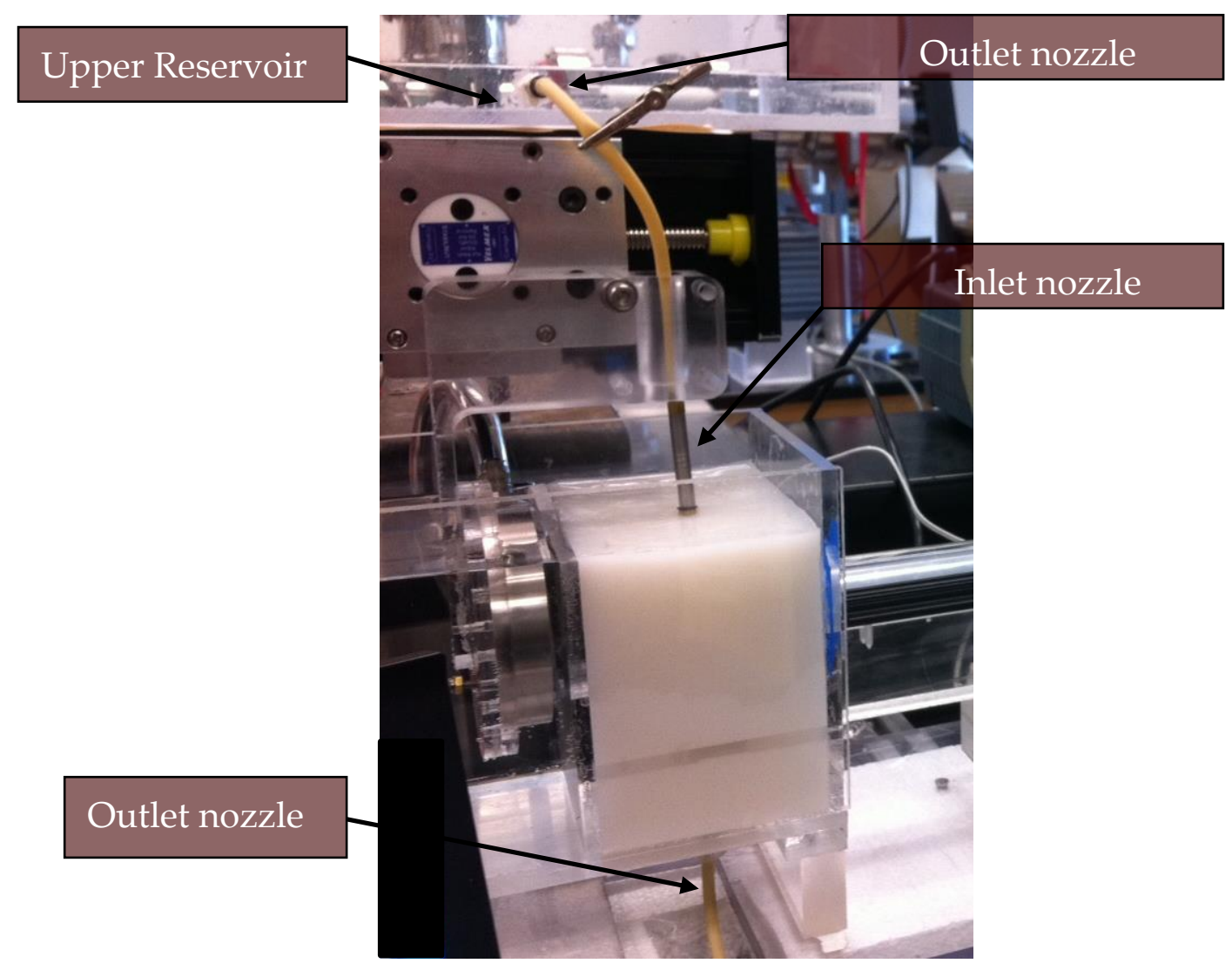

Figure 16. Reservoir and flow system setup

In order to mimic the flow and thermal properties of blood, a bloodmimicking fluid (BMF) was used. Many different solutions have been used by 
researchers, but the simplest BMF is a mixture of degassed water $(99.9 \%$ by weight) and corn starch (0.1\% by weight) [39]. One downside of this BMF is that the corn starch particles tend to settle to the bottom of the reservoir. This issue was mitigated by placing the upper reservoir that held the bulk BMF on top of a magnetic mixer that maintained even particle distribution and prevented clumping.

The HIFU source used in this investigation was an $\mathrm{H}-1011.13 \mathrm{MHz}$ transducer with an external RF impedance matching network (Sonic Concepts, Bothell, WA). The transducer also features a " $3^{\text {rd }}$ harmonic" driving frequency at 3.2 $\mathrm{MHz}$ (not used in this research). The depth of the focal point is $51.74 \mathrm{~mm}$ from the "exit plane of the transducer housing rim..." [40] The driving signal passes through the impedance matching network before being routed to the transducer.
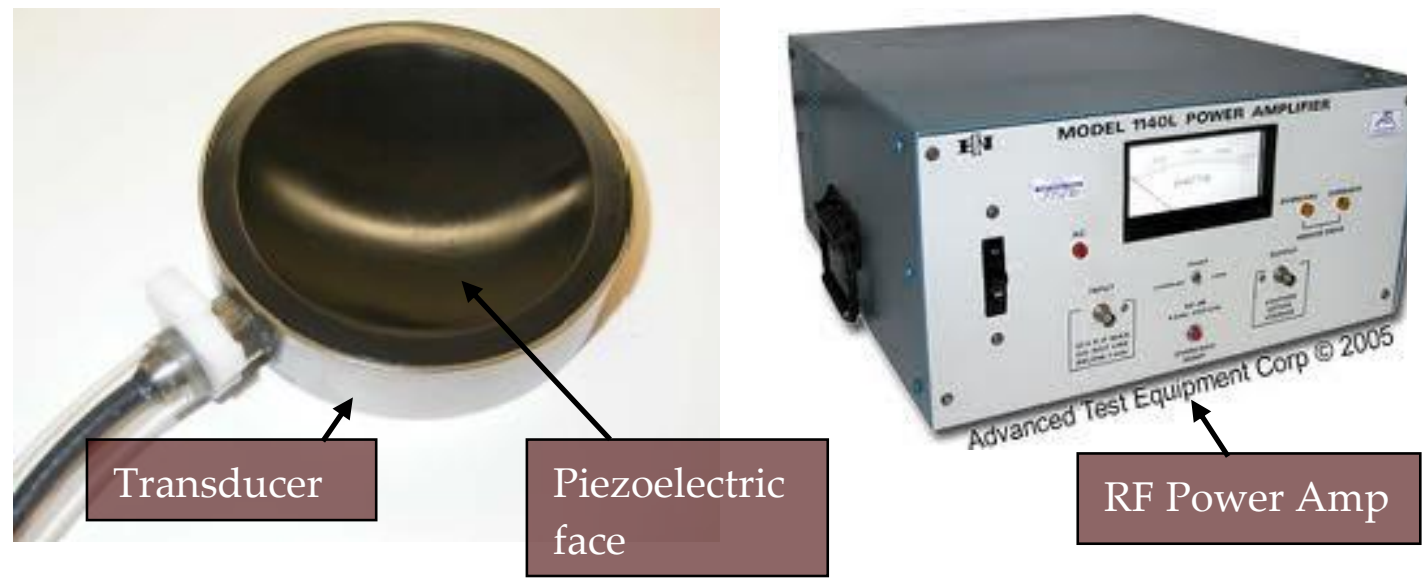

Figure 17. H-101 Transducer and power system 
The driving signal was generated by a function generator and amplified by a RF power amplifier (150 watt, $50 \Omega$ ). The function generator can be set to continuous mode or pulse mode, depending on the application. This investigation used continuous mode for 30 seconds per sonication.

In order to accurately position the HIFU focus within the opaque phantom, a combination imaging-positioning system was implemented. The HIFU transducer was attached by a rigid arm to a tri-axis motor system, which could be moved with a computer controller. Attached to the same fixture, located directly above the phantom vessel, was an ultrasound imaging transducer (V312-SU, Panametrics Transducers). The transducer has a focus range of $11.7 \mathrm{~mm}$ to $53.3 \mathrm{~mm}$ and operates at $10 \mathrm{MHz}$. The tri-axis motors were moved via a LabVIEW program that allows the user to move in increments as small as $0.1 \mathrm{~mm}$. The motor system was located above an ultrasound tank filled with degassed water and the transducer sat below the water line. Figure 18 shows the motor, dual-transducer fixture and ultrasound water tank setup. 


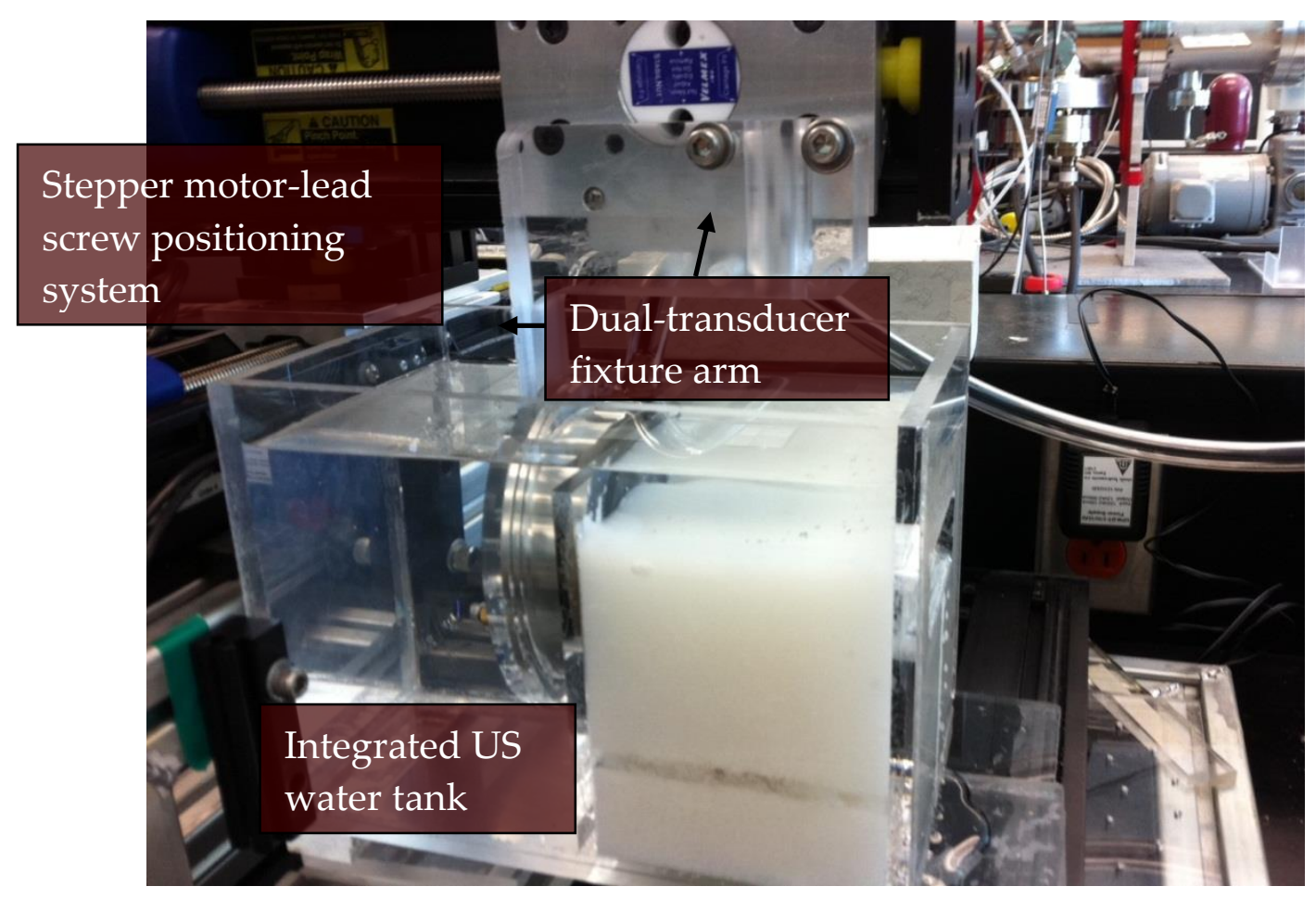

Figure 18. Tri-axis motor system and water tank setup

By immersing the HIFU source in degassed water, the transducer can be positioned horizontally away from the surface of the phantom without losing a significant amount of acoustic energy through attenuation or reflection. Water has very low ultrasound attenuation and acts like a coupling material between the transducer face and the TMM.

The compression apparatus was positioned opposite the HIFU transducer to supply compression through the dedicated window in the mold. The acrylic compression arm was secured to a linear rail (Hayden Kerk, Waterbury, CT) and controlled by a micro-controller (Arduino Uno), as shown in Figure 19. 


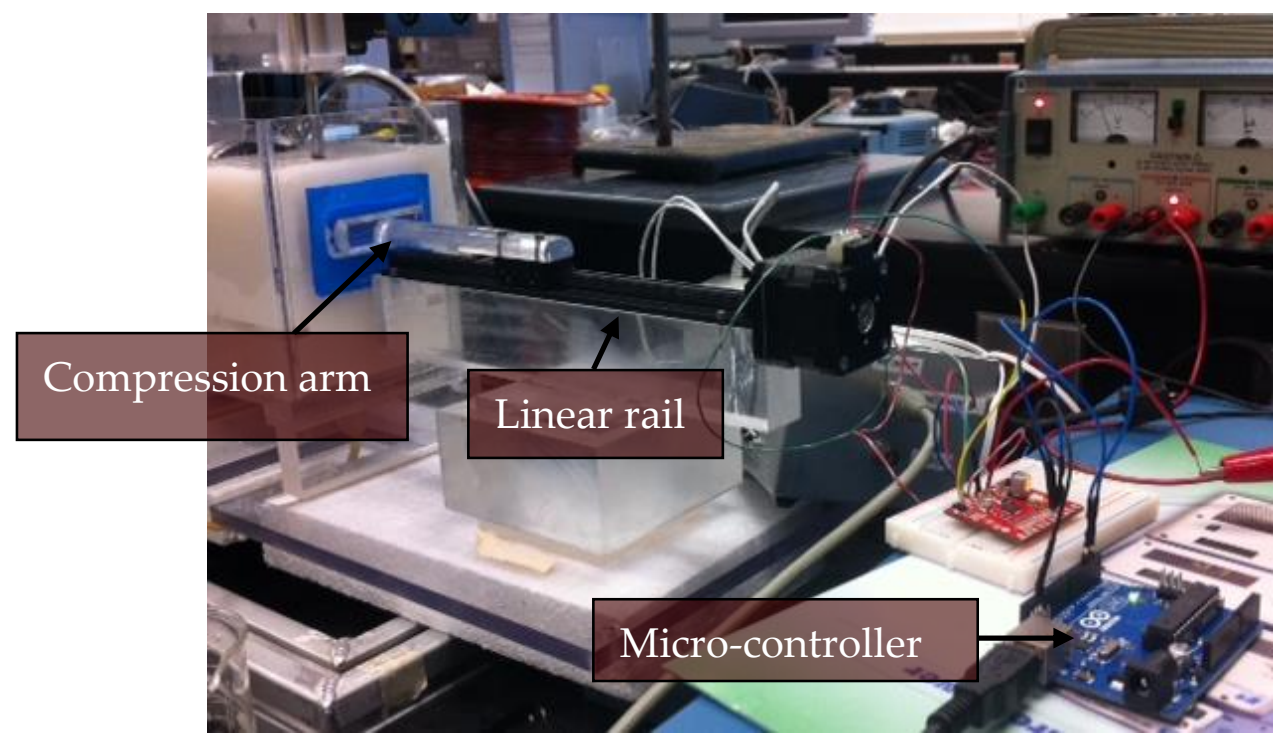

Figure 19. Compression arm, linear rail and micro-controller

The linear rail has a size 17 stepper motor with a thread length of 2.54 $\mathrm{mm}$, a stroke length of $203 \mathrm{~mm}$ and a maximum load of 156 newtons. [41]

For measuring the internal temperatures during heating, precise positioning of the thermocouple probes (relative to the HIFU focus) was essential. The thermocouple fixture was built using sheathed metal hypo-tubes ( $0.89 \mathrm{~mm}$ inner diameter, $0.97 \mathrm{~mm}$ outer diameter) protruding from the acrylic mold and into the phantom. The spacing of the thermocouples relative to the HIFU focus is illustrated in Figure 20. 


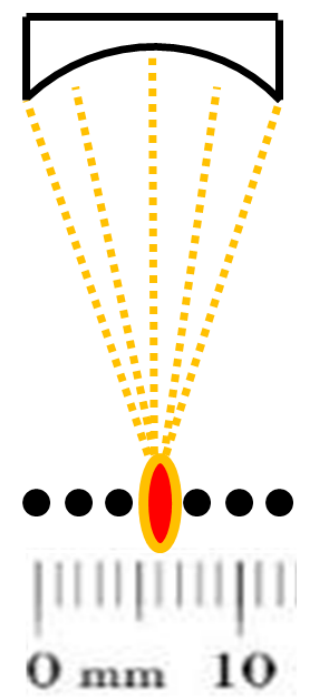

Figure 20. Thermocouple spacing relative to HIFU focus (in red)

The thermocouples used are T-type thin-wire models and the data was acquired using an 8-channel device (TC-08, PICO Technologies, St. Neots, Cambridgeshire, UK). The thermocouples were potted in the hypo-tubes using Loctite 4014 adhesive (Loctite, Westlake, OH). The potted hypo-tubes were positioned in the holes drilled into the phantom mold. The thermocouples were positioned at the height of the HIFU transducer focal point (accurate to $0.5 \mathrm{~mm}$ ) and the hypo-tubes were then permanently set using Loctite 4011 adhesive. The final step was to sheath the hypo-tubes with heat-shrink tubing with the purpose of lowering the reflectivity of the probes during sonication. Figure 21 shows the thermocouple setup inside the phantom mold. 


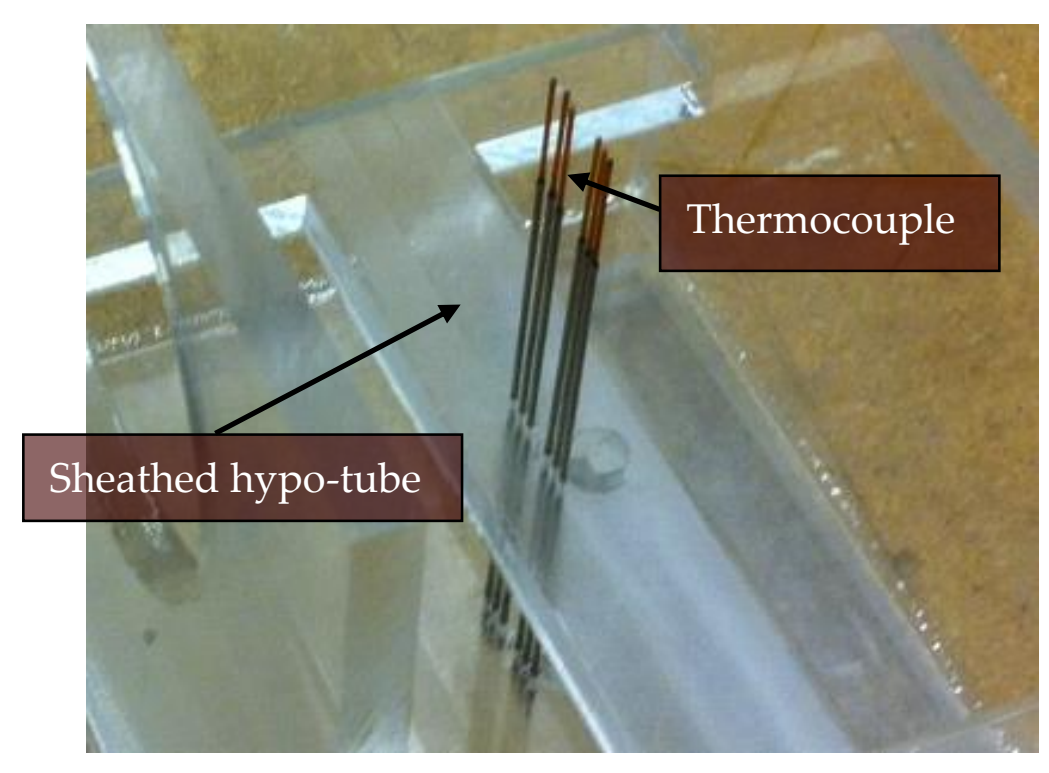

Figure 21. Fixture with thin-wire thermocouples inside sheathed hypo-tubes

\subsubsection{Experimental Plan}

All experiments took place inside an ultrasound water tank located in the Biomedical Systems Lab at SJSU, using the HIFU phantom mentioned in Section 4.11. Prior to running the experiments, every piece of the apparatus was tested and adjusted to meet the requirements designated by the procedure.

The first step was to fill the water tank with degassed, room-temperature water and submerse the phantom. The internal temperature was monitored to ensure that the phantom reached thermal equilibrium before testing commenced. The water tank was fastened to the frame that holds the tri-axis motion system.

For the sake of repeatability, and proper parameter input for the simulations, it was important to know the level and consistency of the acoustic 
power being transmitted by the HIFU transducer. A calibrated ultrasound power meter (UPM-DT, Ohmic Instruments, Easton, MD) was used to measure the acoustic output of the HIFU transducer submersed in a degassed water-filled container. A custom fixture was built to accommodate the H-101 HIFU transducer, holding the face just under the water level. The power meter is shown in Figure 22.

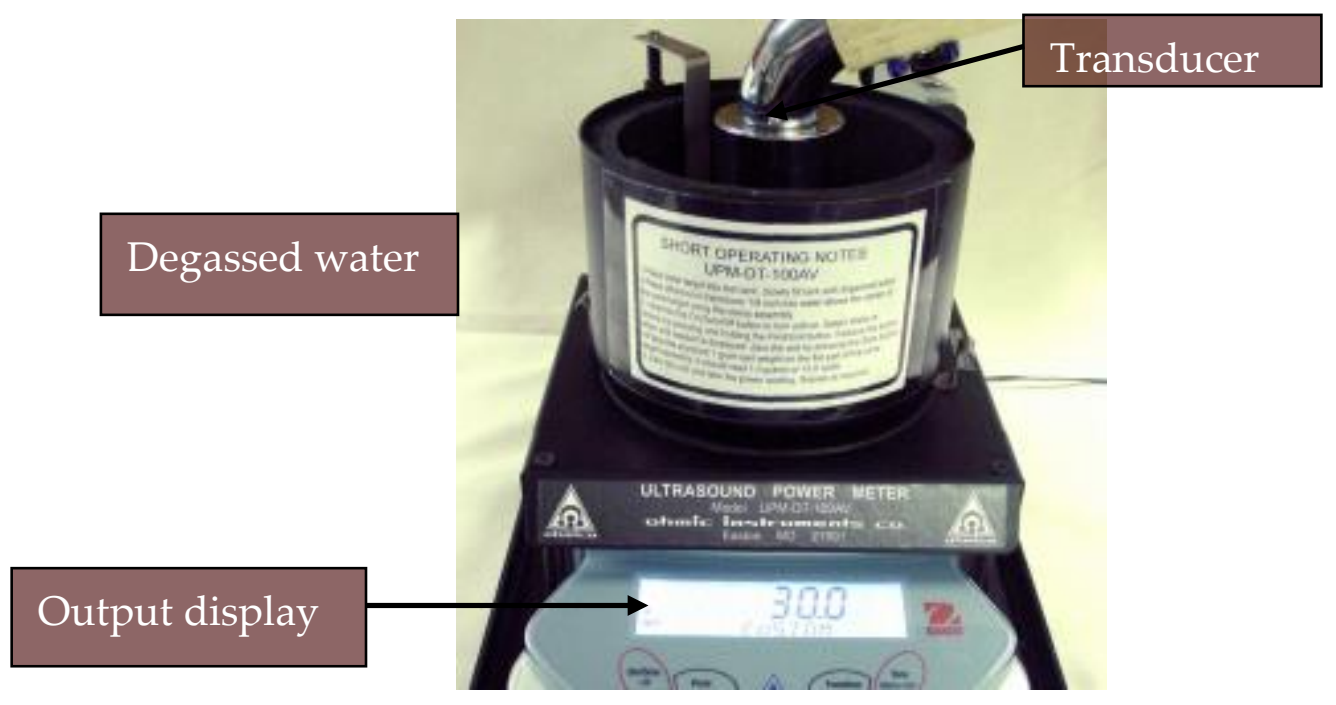

Figure 22. Ultrasound power meter for measuring HIFU transducer output

The power meter was calibrated and zeroed using an object which, when placed on the meter's scale, has a known power output reading (per the manufacturer). Once the meter was ready for sonication, the HIFU transducer and fixture were introduced and the RF amplifier was powered. Allowing at least 5 seconds for the transducer output to level out, the maximum power (shown on the output display, as seen in Figure 22) was recorded over a 30 
second period. This value was used in the numerical simulations and noted in the experimental results.

Another way of measuring the acoustic output of the transducer is directly from the display of the RF amplifier. When the amplifier is transmitting power, it can supply the acoustic output and reflected power using its own internal sensors. A comparison of the two power meters confirmed roughly equivalent readings, making it convenient to use the built-in power meter on the RF amplifier. The amplifier display was used for all experiments. After confirming the transducer performance and amplifier settings, the HIFU transducer and compression-arm were attached to their corresponding fixtures, positioning them relative to the phantom.

In order to compare the temperature profile directly in the phantom with and without compression of the embedded vessel, the HIFU focus needs to be at about the same location in relation to the imbedded target in the phantom. Essentially, the process was meant to mimic the ablation of a tumor that had been displaced slightly by tissue compression. To compensate for the displacement of the "target" region of the phantom, small adjustments of the HIFU focus point must be made that directly follow the displacement that occurs after external compression. This was accomplished with the ultrasound imaging component of the experimental apparatus. 
With the knowledge that reflective metal beads are recognizable on an ultrasound imaging scan, the metal junctions on the ends of the embedded thermocouples serve as effective markers for re-positioning the HIFU transducer after compression. An initial scan of the uncompressed phantom, using the 1-D V312-SU Panametrics transducer, supplied the characteristic ultrasound plot of the embedded thermocouples. For repositioning after compression, the dualtransducer fixture was moved in the horizontal plane until the characteristic plot appeared on the scan. This new location, which is even with the line of thermocouple probes, was set as the new target location for the HIFU transducer. Illustrations of this process and the output of the 1-D ultrasound transducer scan are shown in Figure 23

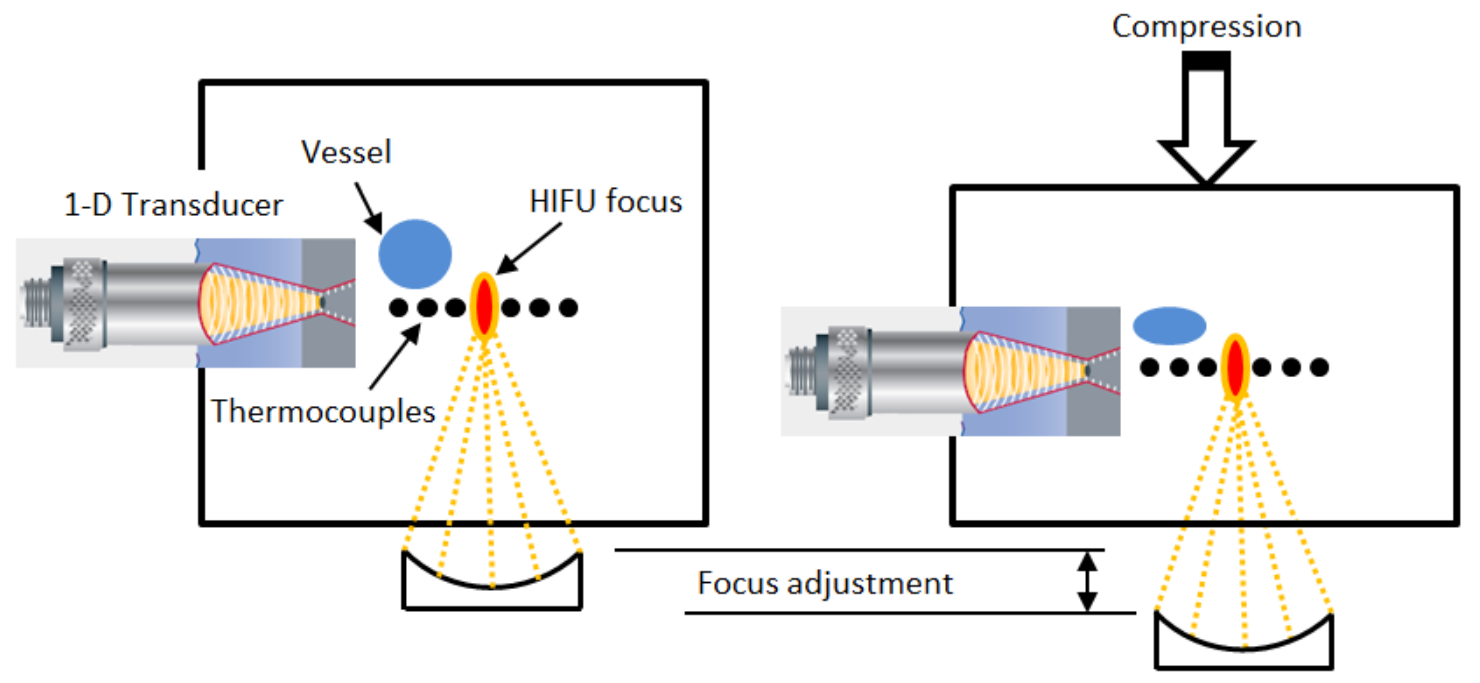

(a) 


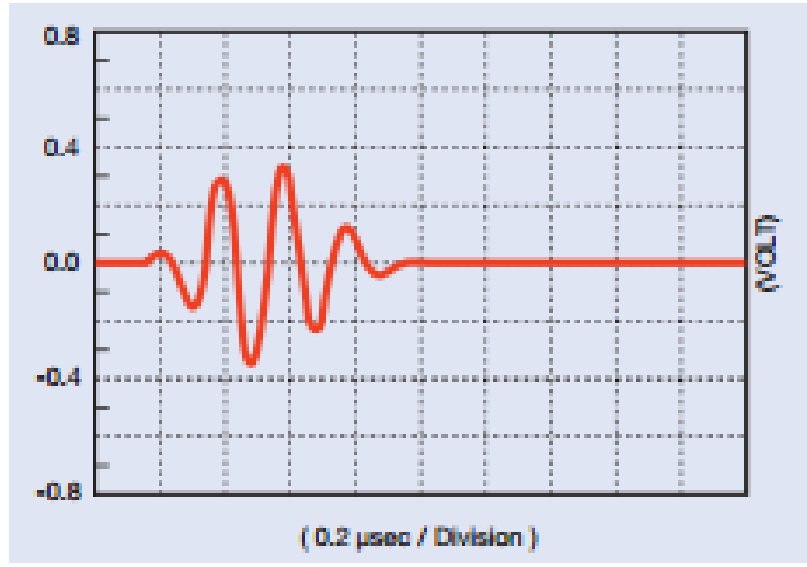

(b)

Figure 23. (a) Illustration of HIFU focus adjustment after compression and (b) example of reflected ultrasound imaging waveform

After the HIFU transducer's focal point was properly positioned, the fluid flow system was connected to the phantom vessel and tuned (see Section 4.2.2) to provide the desired flow rate.

After verifying that the flow rate was in the correct range, the thermocouple system was checked. Six thermocouples were positioned near the HIFU focal point (Section 4.2.2) and were checked to ensure readings near the temperature of the water bath. If any thermocouple readings were more than 5 degrees less than or greater than the temperature of the water bath, they would be replaced before running any experiments.

The experimental parameters included in this investigation include the amount of applied compression and the power of the HIFU driving source. The external compression, increased incrementally, was applied until complete vessel 
occlusion occurred (zero flow out of vessel). The incremental step-size was chosen to be $2.5 \mathrm{~mm}$ ( $0.10 \mathrm{inch})$, based on preliminary experiments, applied at the surface opposite the HIFU transducer (perpendicular to flow). The phantom vessel architecture was designed as a single $6 \mathrm{~mm}$ diameter vessel directly in the center of the phantom, running perpendicular to the plane of the HIFU wave propagation.

An experimental procedure matrix was created to systematically test the effects of varying the parameters listed in this section, shown in Table 4.

Table 4. Experimental Procedure Matrix

\begin{tabular}{|l|l|l|}
\hline \multicolumn{4}{|c|}{ Single-Vessel Tissue Phantom } \\
\hline Power (W) & Compression (mm) & Measured \\
\hline $15 \mathrm{~W}$ & 0 & Temperature \\
\hline $50 \mathrm{~W}$ & 0 & Temperature \\
\hline $30 \mathrm{~W}$ & 0 & Temperature and flow \\
\hline $30 \mathrm{~W}$ & 2.5 & Temperature and flow \\
\hline $30 \mathrm{~W}$ & 5.1 & Temperature and flow \\
\hline $30 \mathrm{~W}$ & 7.6 & Temperature and flow \\
\hline $30 \mathrm{~W}$ & 10.2 & Temperature and flow \\
\hline $30 \mathrm{~W}$ & 12.7 & Temperature and flow \\
\hline $30 \mathrm{~W}$ & 17.8 & Temperature and flow \\
\hline $30 \mathrm{~W}$ & 30.5 & Temperature and flow \\
\hline
\end{tabular}




\begin{tabular}{|l|l|l|}
\hline $30 \mathrm{~W}$ & 33.0 & Temperature and flow \\
\hline $30 \mathrm{~W}$ & 35.6 & Temperature and flow \\
\hline $30 \mathrm{~W}$ & 38.1 & Temperature and flow \\
\hline $30 \mathrm{~W}$ & 40.6 & Temperature and flow \\
\hline
\end{tabular}

The temperatures at each sensor location was recorded for the duration of sonication (30 seconds) and exported to MATLAB for plotting and postprocessing. This is repeated for each run designated in the experimental procedure matrix. For example, temperature data was collected for a $30 \mathrm{~W}$ sonication of the single-vessel phantom with $2.5 \mathrm{~mm}$ ( 0.10 inches) of surface displacement and the phantom was allowed to cool back to room temperature. Then the experiment was repeated with $0.51 \mathrm{~mm}$ (0.2 inches) of displacement.

A major advantage of using the gellan gum phantom approach is that the phantom is totally reusable. All the experiments were done with the same phantom without having to move the setup or replace the thermocouple fixture. Extreme caution was exercised ensuring that the HIFU focus and thermocouple placements were as precise as possible, a difficult task. One-dimensional ultrasound imaging was a crucial tool for maintaining consistency.

During sonications, the thermocouples were sampled at $2 \mathrm{~Hz}$, meaning 2 data points were collected every second. High resolution of the temperature 
data was not required for this investigation since overall heating uniformity was the main concern. The data was streamed via a USB data acquisition device. The complete temperature data for each thermocouple was exported as a text document and loaded into a MATLAB program (Appendix B) which plotted the temperature data versus time. Figure 24 shows an example of a MATLAB temperature plot for thermocouples at 2, 4, and $6 \mathrm{~mm}$ from a HIFU heat source.

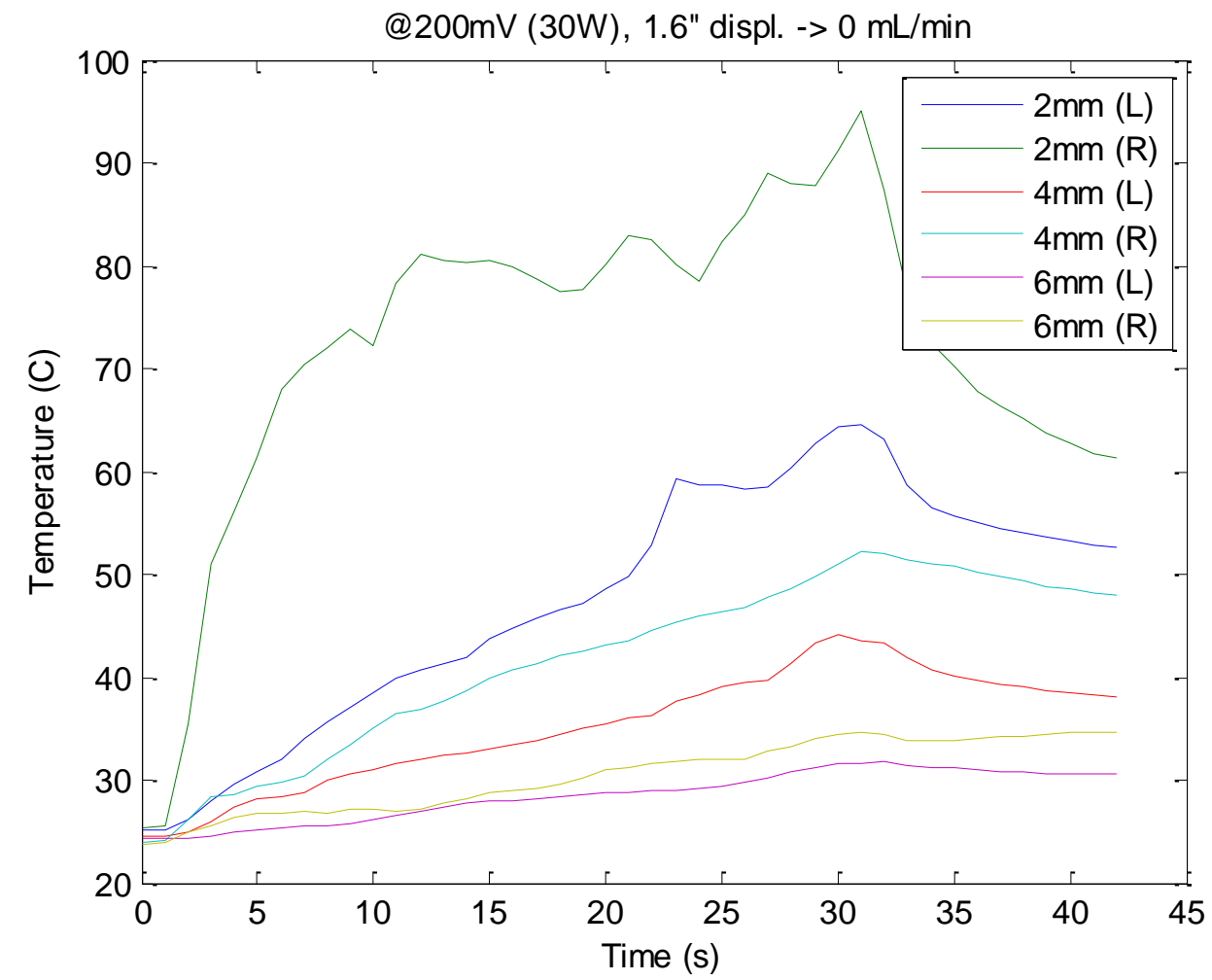

Figure 24. Plot of acquired temperature data at various depths in phantom (MATLAB) 


\subsubsection{Heating Uniformity Metric}

The degree of change in spatial heating uniformity during phantom compression is ultimately the gauge for agreement between the results of this investigation and the hypothesis. For both the computational and experimental aspects of this investigation, the degree of uniformity had to be quantifiable.

Temperature data was collected at various depths along the radial plane, so the temperature deviations between those different locations were calculated. The difference in temperatures at each depth was measured:

$$
d T=a b s\left(T_{2 m m, l e f t}-T_{2 m m, r i g h t}\right)
$$

where $T_{2 m m \text {,left }}$ represents the temperature measured at $2 \mathrm{~mm}$ depth on the left side of the HIFU focus.

For each proposed run listed in the experimental procedure matrix specified in Table 4, the left vs. right temperature difference was calculated at each data probe depth. The smaller the temperature difference, the more uniform the heating. The maximum and minimum temperatures (hot and cold spots) were also analyzed and compared for all the runs. It seems important to examine uniformity as well as potentially dangerous (above $100{ }^{\circ} \mathrm{C}$ ) hot and cold (below $50{ }^{\circ} \mathrm{C}$, the approximate temperature threshold for ablation) spots in the phantom. 
The simulation results were analyzed in exactly the same manner, although the metrics were calculated within the MATLAB program. Since the temperature for each location in the simulated material grid were calculated and stored, it was easy to calculate the temperature differences to compare with the experimental results.

\subsection{Safety of Experiments}

Almost all of the safety concerns for this research relate to the production of the gellan gum phantoms, although some danger exists in running the HIFU transducer. The ingredients include many small particles, such as the aluminum oxide, calcium chloride and potassium sorbate particles. The Material Safety Data Sheets (MSDS) for these ingredients list inhalation of particles as a potential hazard. To limit exposure, all handling of phantom ingredients was done in a chemical fume hood with proper remote exhaust to keep the particles from escaping into the lab. In addition, gloves and face masks were worn as secondary personal protective equipment (PPE) beyond the fume hood.

The phantom production process also involved the use of a hot plate and vacuum chamber (in this case, a filter flask with vacuum-hose nozzle). Standard precautions were taken to avoid accidentally touching the hot plate or any of the heated beakers/flasks without hand protection. Heat-resistant gloves were used to handle any hot containers. The vacuum chamber had the potential to shatter 
when placed under vacuum, so a protective cage was put over the filter flasks during de-pressurization. After the TMM was poured into the mold and congealed, it was kept in a sealed plastic bag to prevent unnecessary exposure to the material.

A few hazards were present during the actual experimental procedure. Because all phantom experiments were conducted inside a water bath, care was taken to avoid exposure of electrical components to the water. The HIFU transducer, which was submersed in water, was fitted with a water-proof tube that protects the RF power cable from contacting the surrounding water. This integrity of the tubing was checked prior to every experimental run. In addition, the HIFU transducer was turned off whenever it was not being used for an ablation run. This prevented unnecessary exposure to the ultrasonic waves which can burn skin in the focal region. 


\section{CHAPTER FIVE \\ RESULTS AND DISCUSSION}

This thesis investigation had two goals: to build a tissue-mimicking material phantom, with a fluid vessel and embedded thermocouples, that was mechanically, thermally and acoustically similar to soft-tissue, and to determine the effects of compression on convection and heating uniformity during HIFU ablation.

Early fabrication attempts included trying to use standard gelatin (often used for acoustic imaging phantoms) and attempting to find recipes for the polyacrylamide/BVA gel that costs hundreds (to thousands) of dollars. The gellan gum phantom turned out to have the most soft-tissue-like acoustic heating properties and its reusability was very important for this investigation. Techniques were learned and improved upon that led to the successful fabrication of multiple walls-less vessel phantoms with precisely positioned thermocouples. Improvements in the thermocouple sheathing process can also be viewed as an improvement over previous designs described in the literature.

While the fabrication of a useful HIFU phantom with vessel was achieved, there was a major shortcoming in the mechanical properties of the TMM. An important part of this thesis was the relationship between compression and flow through the internal vessel. Fabrication of earlier phantoms made with standard 
gelatin provided products that were very similar to soft tissue in their mechanical properties. Stiffness and elasticity of these earlier phantoms were close to data reported for soft breast tissues. In comparison, the gellan gum phantoms were much stiffer and less elastic than the preceding phantoms. Ultimately the thermal and acoustical properties were deemed most important for the experiments. Ideally compression would have been increased until significant changes occurred in the vessel flow rate, but there was observed deterioration of the phantom in the compressed region. In the future, attempts should be made to alter the recipe to achieve more elasticity and less stiffness. An expected relationship between compression and flow rate did appear, decreasing from $40 \mathrm{~mL} / \mathrm{min}$ with no compression to no flow (total occlusion of the vessel) at $40.6 \mathrm{~mm}$ (1.6 inches) of compression. The averaged data collected over the two days of experimentation are summarized in Figure 25. 


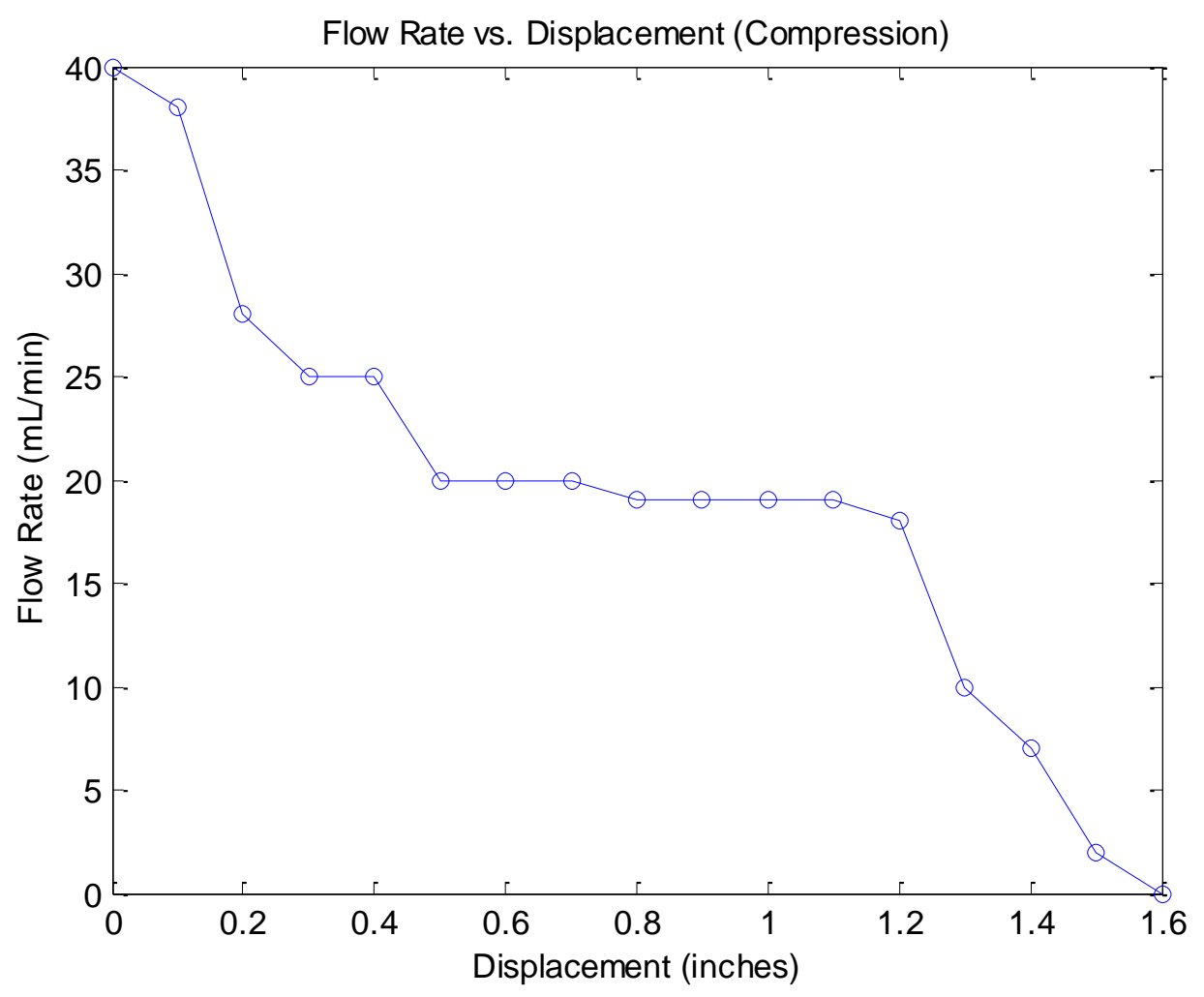

Figure 25. Relationship between displacement of the phantom wall and flow rate

\subsection{Experimental Results}

The data presented in this section were collected on two separate days using the same phantom and same general experimental setup. After the raw temperature data was collected, it was processed using MATLAB. The data were smoothed using a time-averaging method. As a reminder of the locations of the thermocouples in relation to the fluid vessel and HIFU focus, Figure 26 illustrates the experimental setup. 


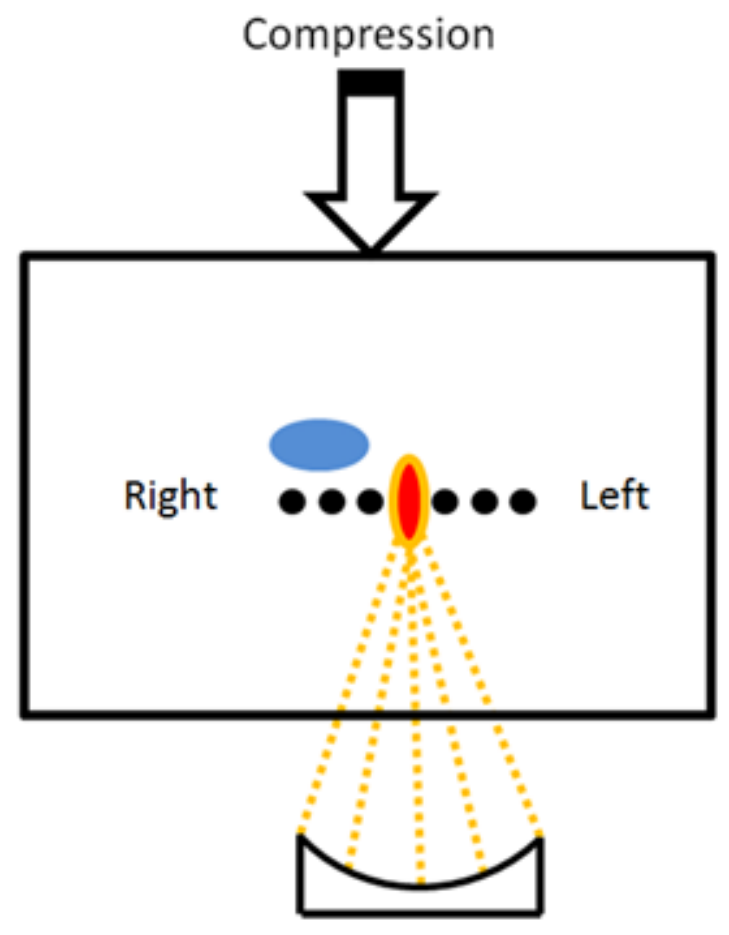

Figure 26. Thermocouple locations relative to the HIFU focal point and vessel

The HIFU system was calibrated (such that generator display output power matched the acoustic power meter). The function generator was set at a frequency of $1.13 \mathrm{MHz}$ and the peak-to-peak voltage $\left(\mathrm{V}_{\mathrm{pp}}\right)$ was adjusted until the desired heating was achieved in the phantom with no flow and no compression. The optimal setting turned out to be $200 \mathrm{mV}_{\mathrm{pp}}$. The sequential temperature plots, starting with no compression and increasing to $40.6 \mathrm{~mm}$ (1.6 inches) of compression, are shown in Figure 27. 


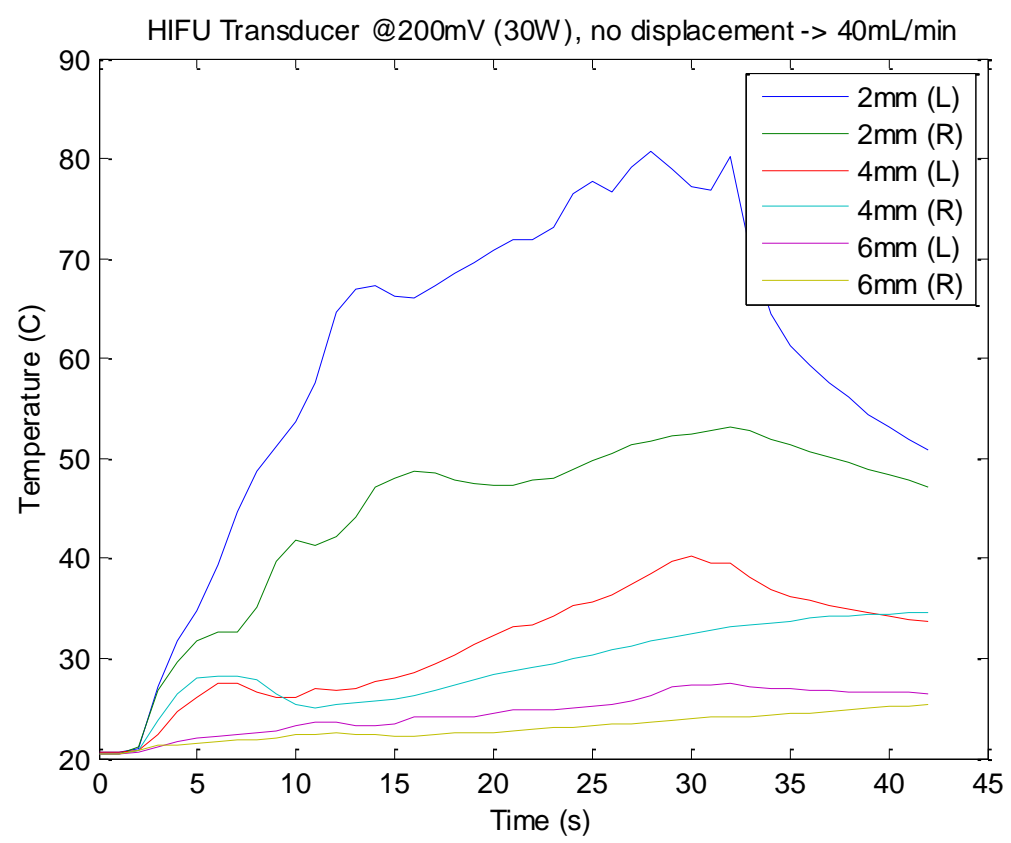

Figure 27 (a) No compression and $40 \mathrm{~mL} / \mathrm{min}$ through vessel

As expected, for each thermocouple pair ( $2 \mathrm{~mm}, 4 \mathrm{~mm}, 6 \mathrm{~mm})$, the left-side thermocouple achieved higher temperature than its counterpart on the right. The thermocouples on the right experienced convective cooling through the fluid vessel which was in near proximity. This trend, which was expected based on theory and predicted temperature profiles, remains true until the flow reaches $25 \mathrm{~mL} / \mathrm{min}$. For all flow rates below 25 $\mathrm{mL} / \mathrm{min}$, the right-side temperatures unexpectedly remain higher than those on the left side. 


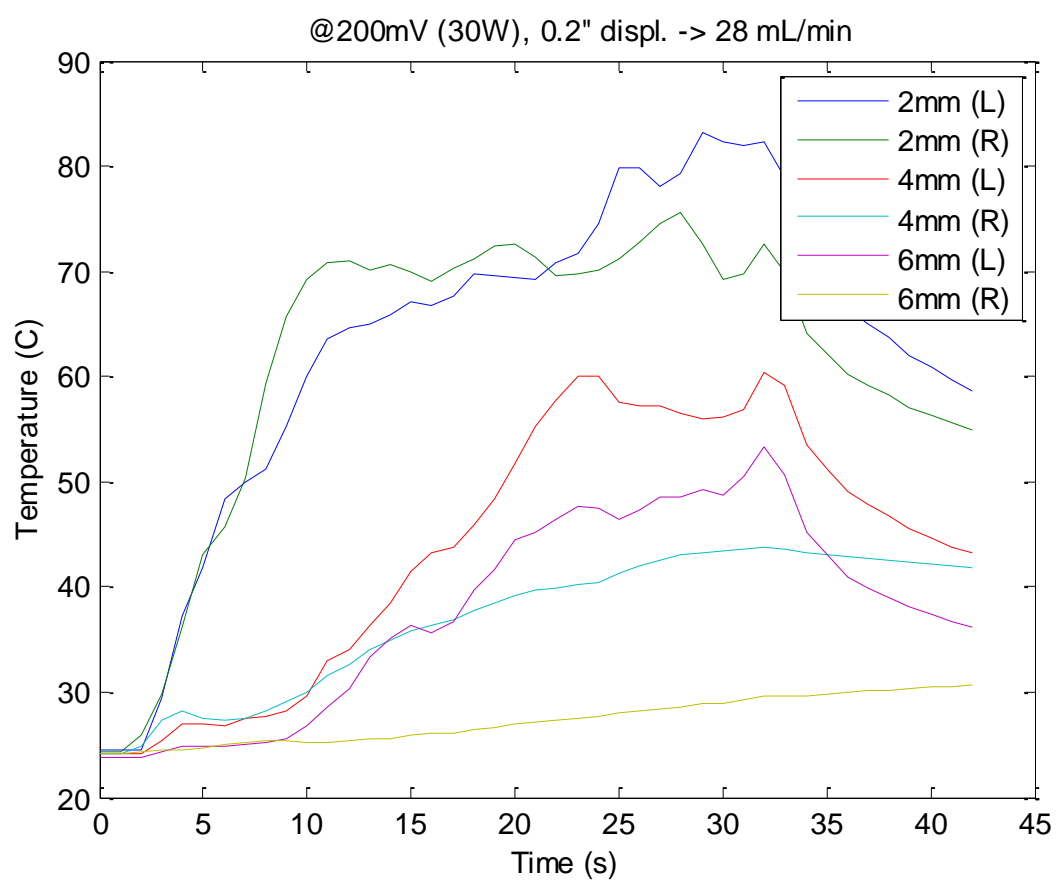

(b) $\sim 5 \mathrm{~mm}$ compression and $28 \mathrm{~mL} / \mathrm{min}$ through vessel

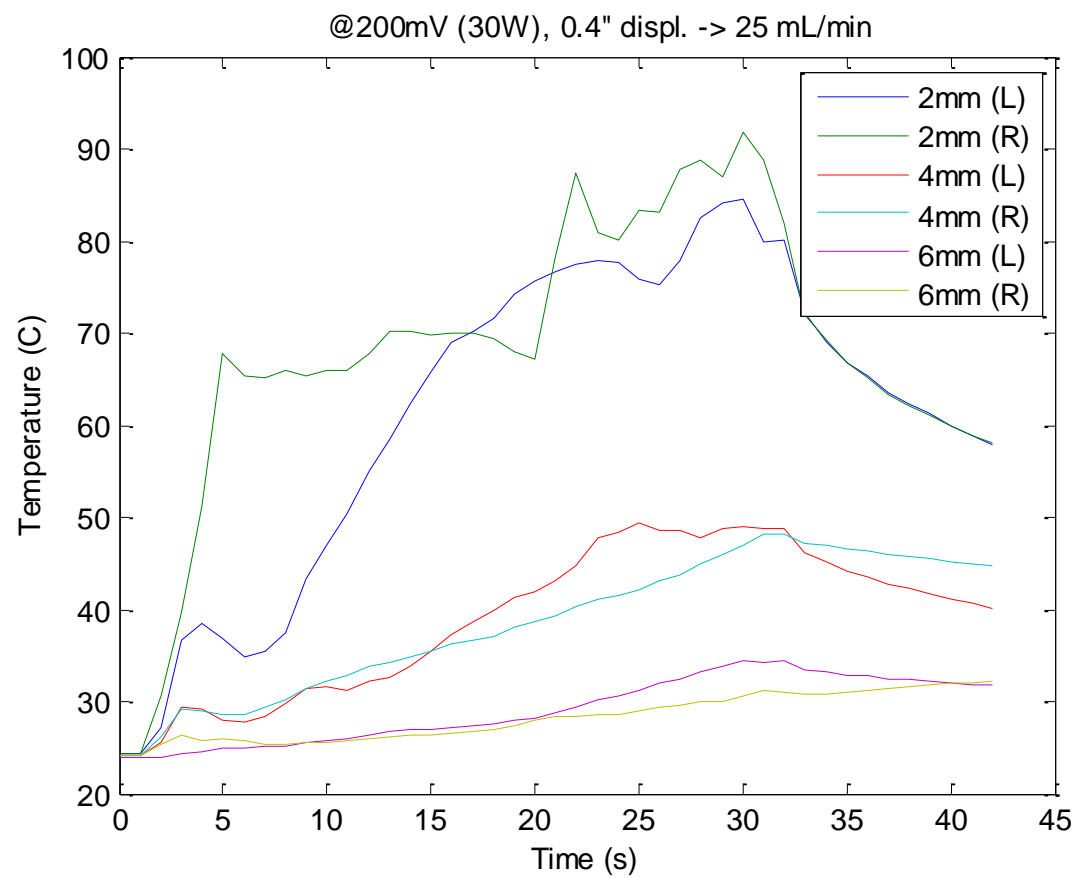

(c) $10.6 \mathrm{~mm}$ compression and $25 \mathrm{~mL} / \mathrm{min}$ through vessel 


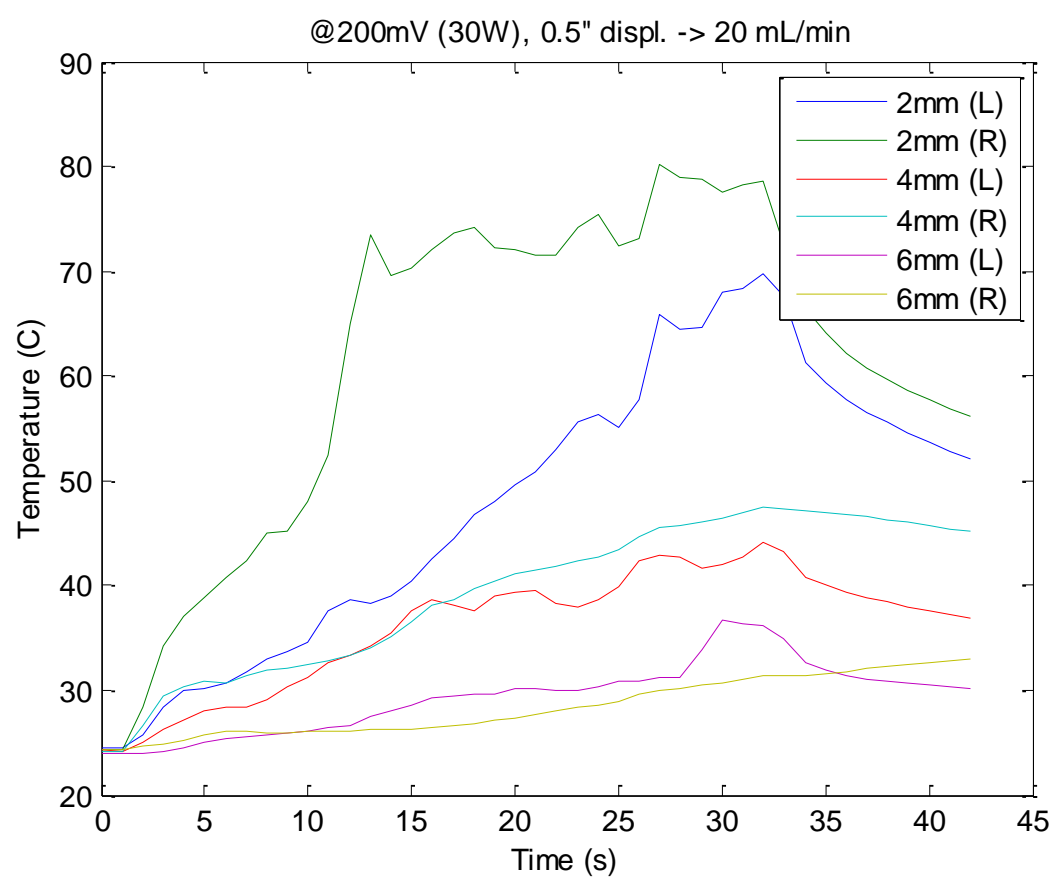

(d) $12.7 \mathrm{~mm}$ compression and $20 \mathrm{~mL} / \mathrm{min}$ through vessel

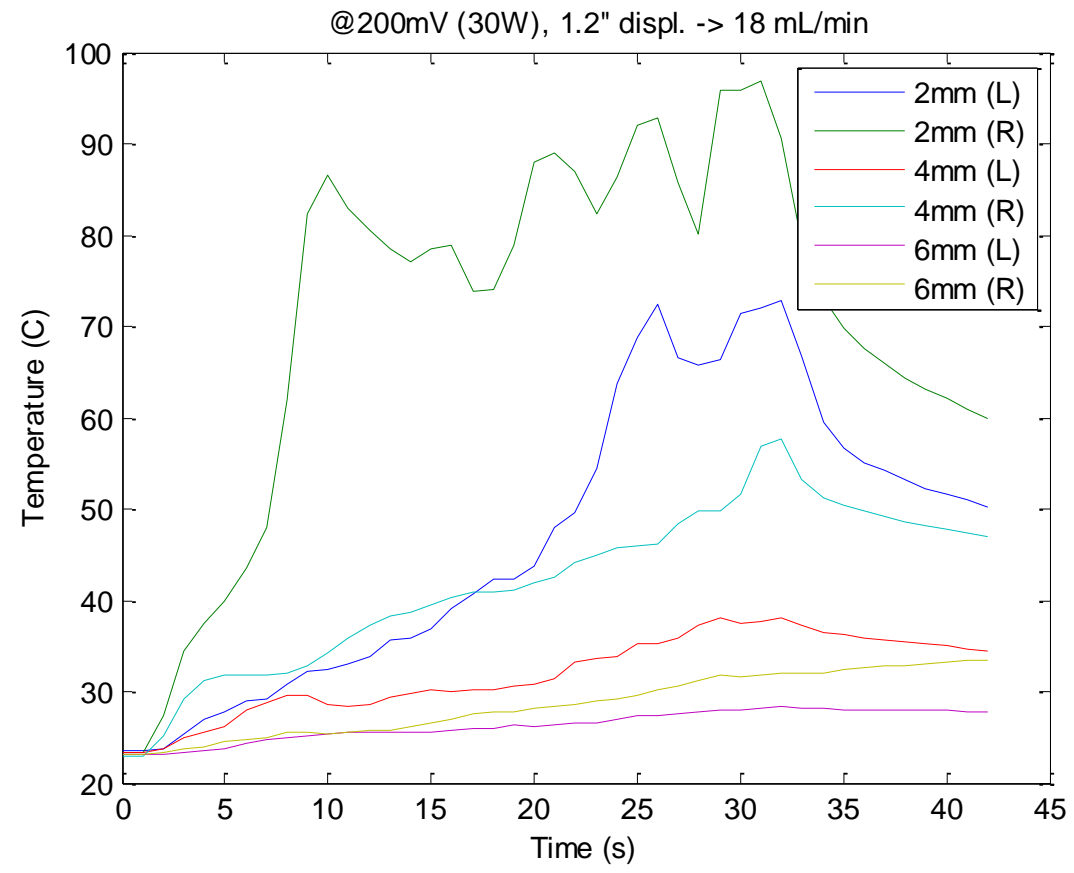

(e) $\sim 30.5 \mathrm{~mm}$ compression and $18 \mathrm{~mL} / \mathrm{min}$ through vessel 


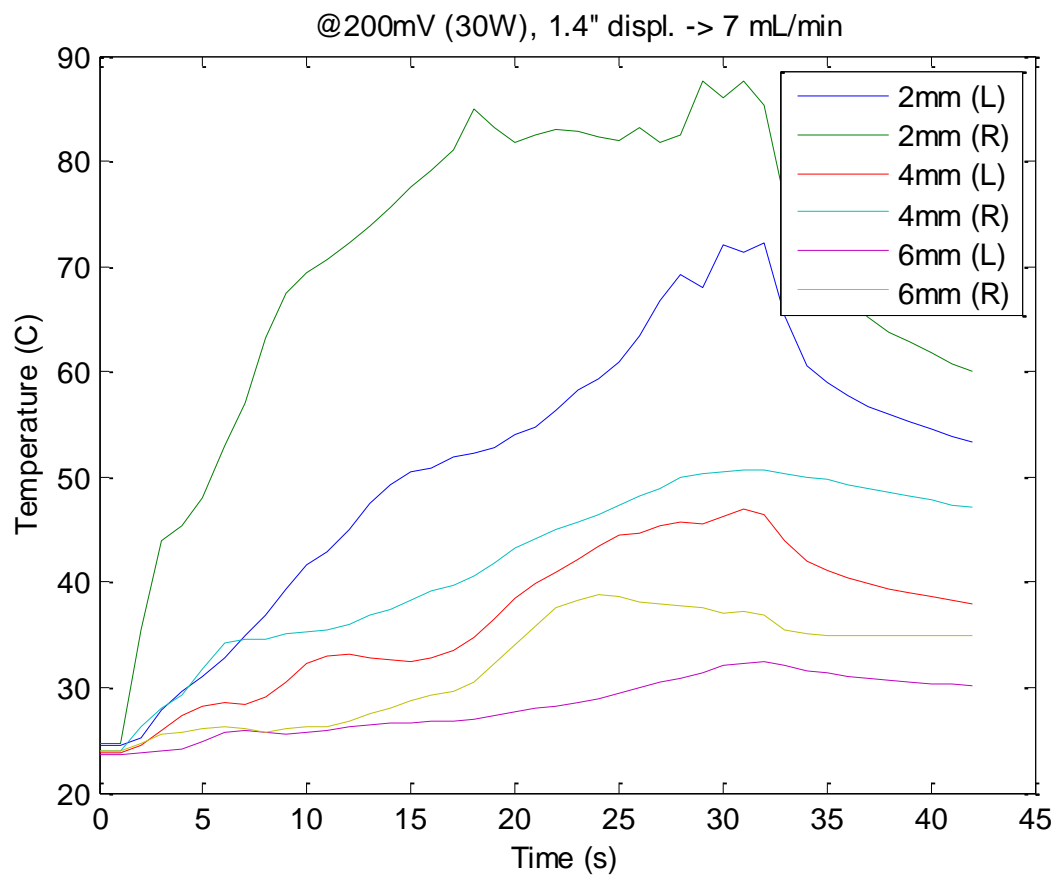

(f) $\sim 35.6 \mathrm{~mm}$ compression and $7 \mathrm{~mL} / \mathrm{min}$ through vessel

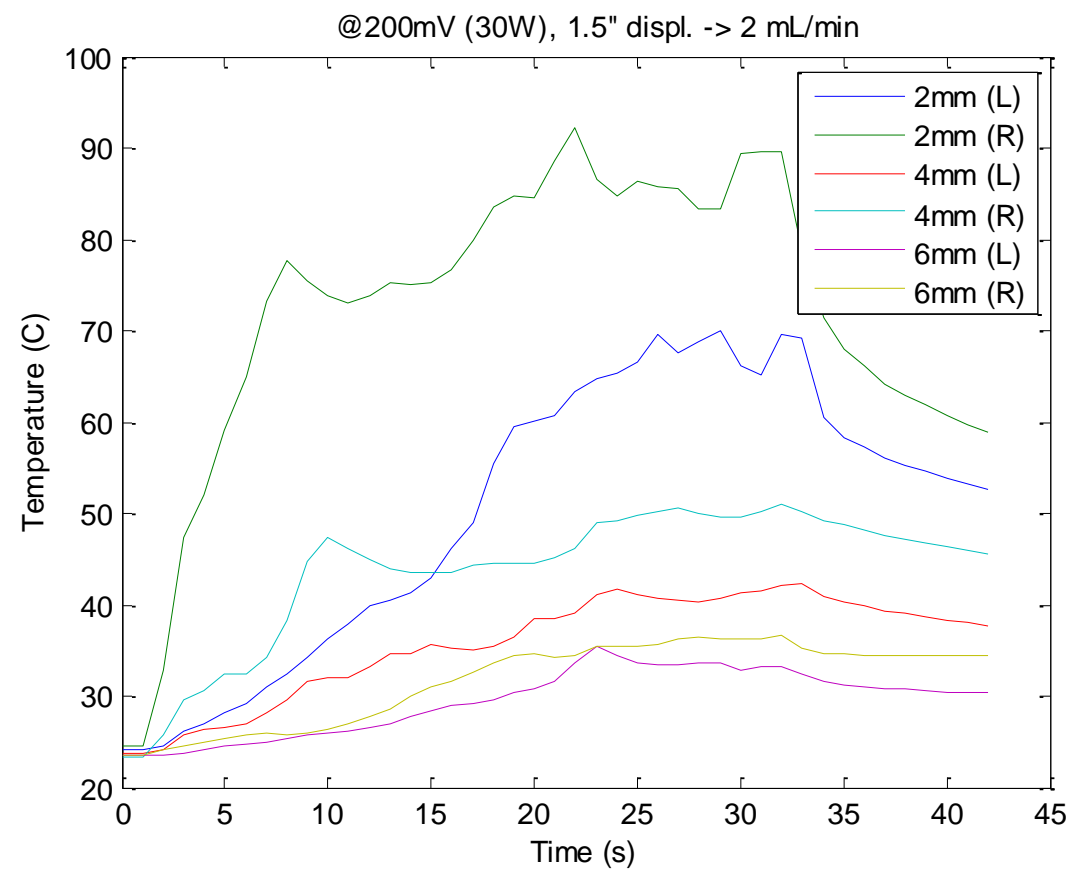

(g) $38.1 \mathrm{~mm}$ compression and $2 \mathrm{~mL} / \mathrm{min}$ through vessel 


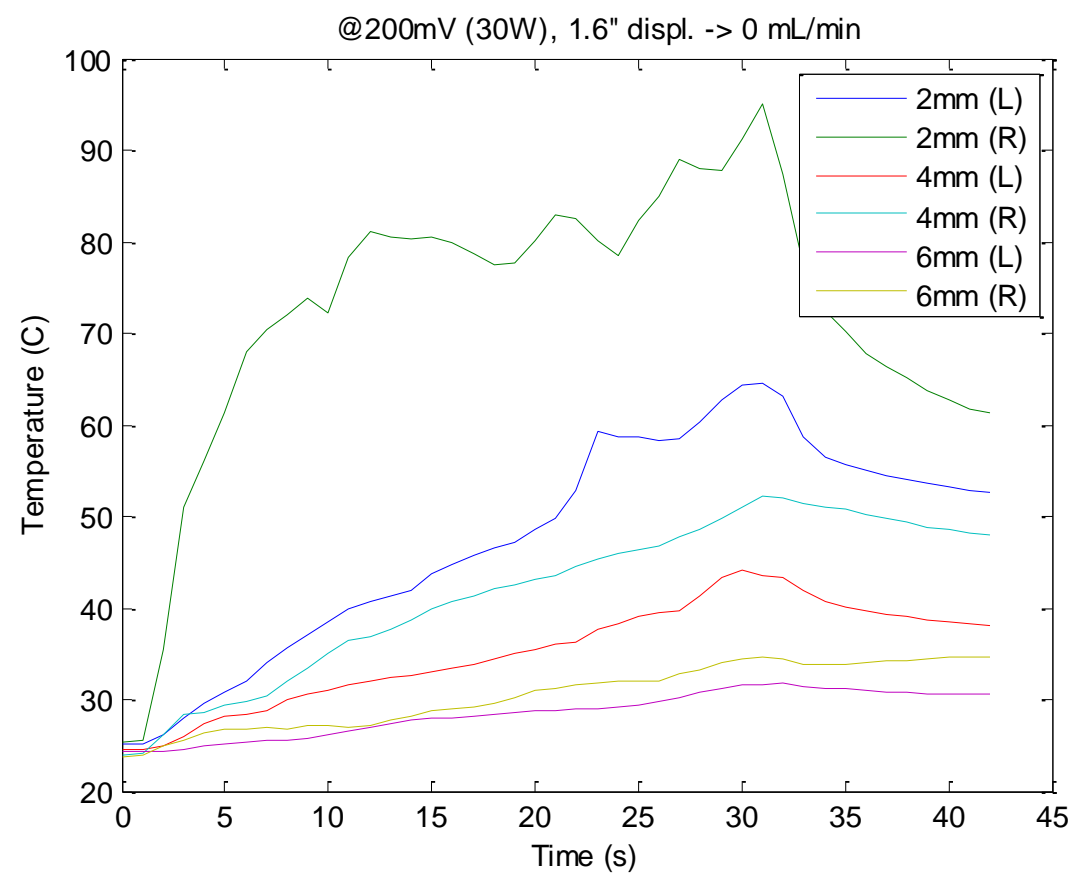

(h) $40.6 \mathrm{~mm}$ compression and total vessel occlusion $(0 \mathrm{~mL} / \mathrm{min})$

Figure 27. Raw temperature plots at each thermocouple for all flow rates

The change in temperature at $2 \mathrm{~mm}$ was of major interest, especially on the right side of the HIFU focus (designated ' $+2 \mathrm{~mm}$ ', as opposed to ' $-2 \mathrm{~mm}$ ' on the left side) where the vessel was located. The transient temperature plots at +2 $\mathrm{mm}$ and $-2 \mathrm{~mm}$ were studied at each flow rate to look for obvious changes as the vessel was compressed. A comparison of transient $+2 \mathrm{~mm}$ and $-2 \mathrm{~mm}$ temperature plots for various flow rates are shown in Figure 28. 


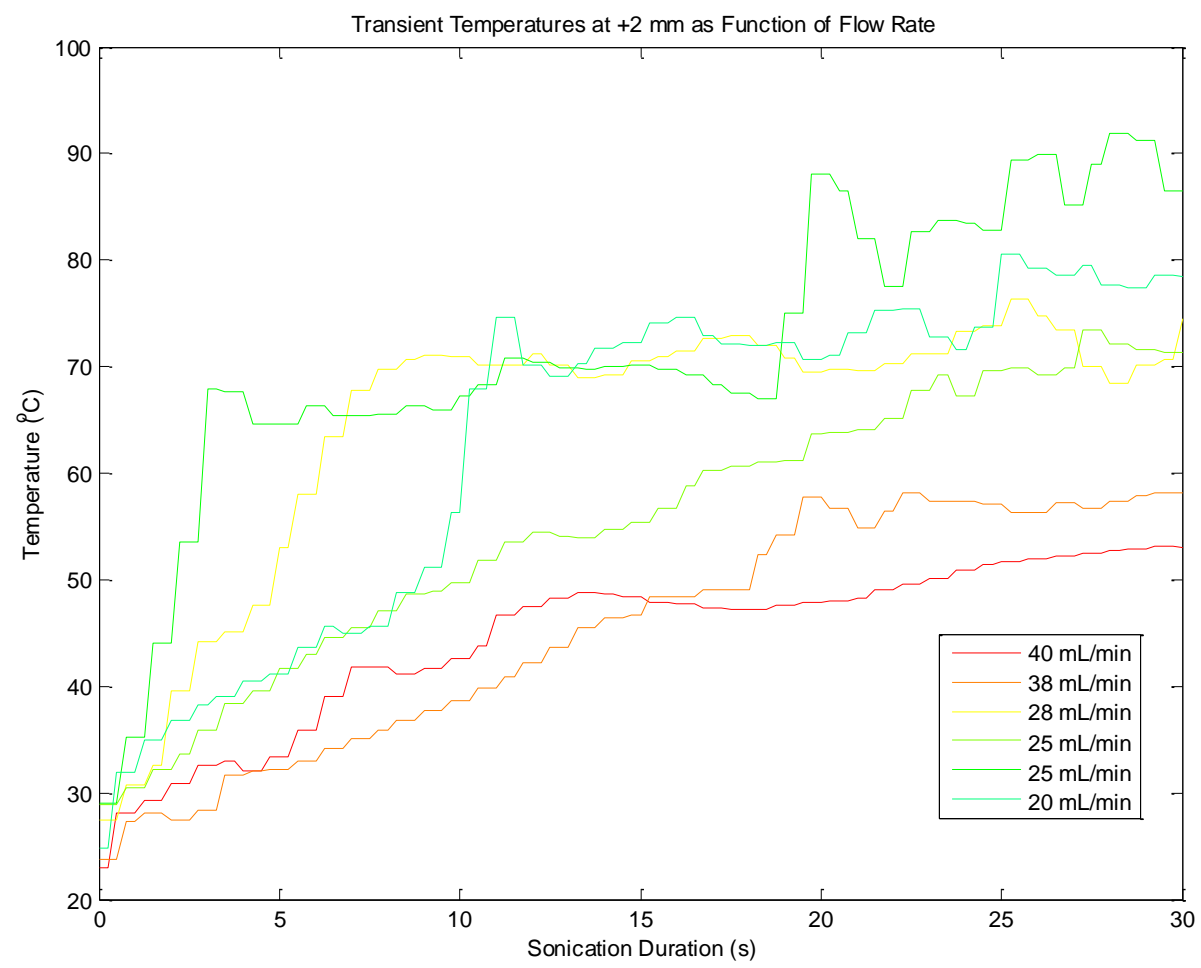

(a)

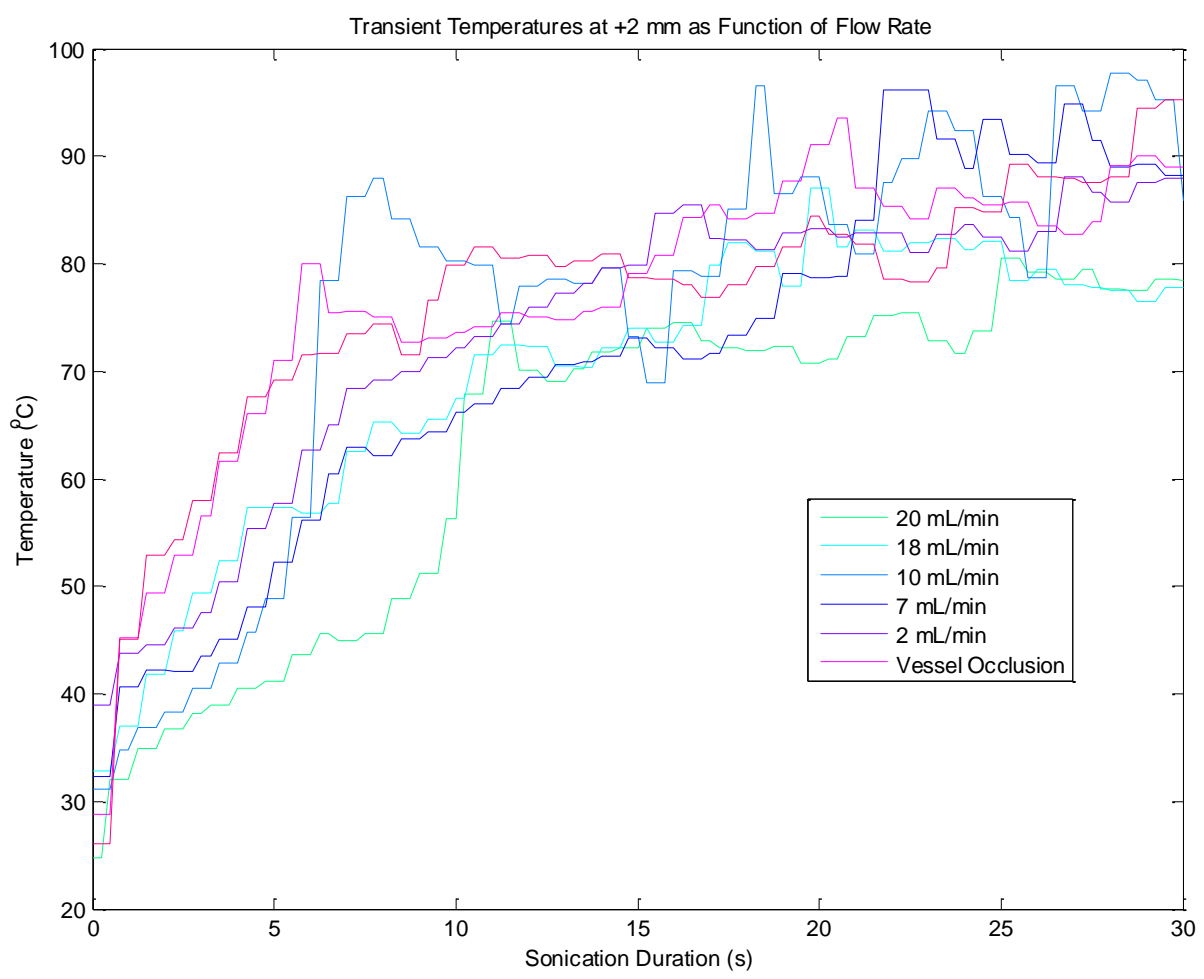

(b)

Figure 28. Transient temperature plots at $+2 \mathrm{~mm}$ for flows between (a) 40-20 $\mathrm{mL} / \mathrm{min}$ and (b) $20-0 \mathrm{~mL} / \mathrm{min}$ 
The transient temperature plots at $+2 \mathrm{~mm}$ show a consistent trend of increasing temperatures as the flow rate decreases through the vessel. The changes in the transient temperature slope and peak temperature are the most obvious as the flow transitions from $40 \mathrm{~mL} / \mathrm{min}$ (no compression on the material) to $20 \mathrm{~mL} / \mathrm{min}$ (12.7 $\mathrm{mm}$ of compression).

It is useful to compare the heating behavior, particularly on the right side at $+2 \mathrm{~mm}$, to the manner in which the flow rate changes with compression. This relationship is plotted in Figure 29.

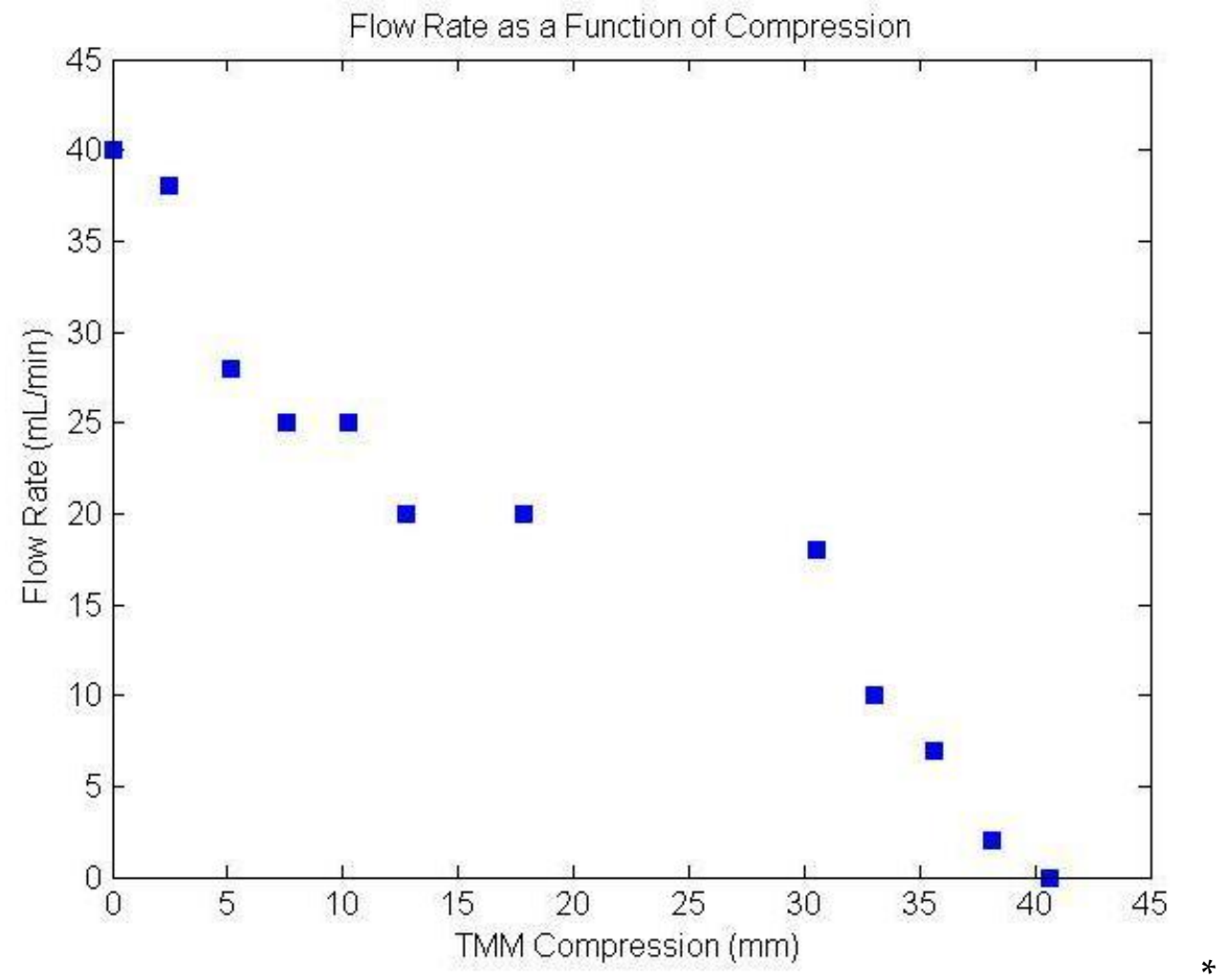

Figure 29. Plot of flow rate versus compression of the tissue-mimicking material 
The plot of maximum temperatures (after 30 seconds of heating) at $+2 \mathrm{~mm}$ as a function of flow rate through the vessel is shown in Figure 30.

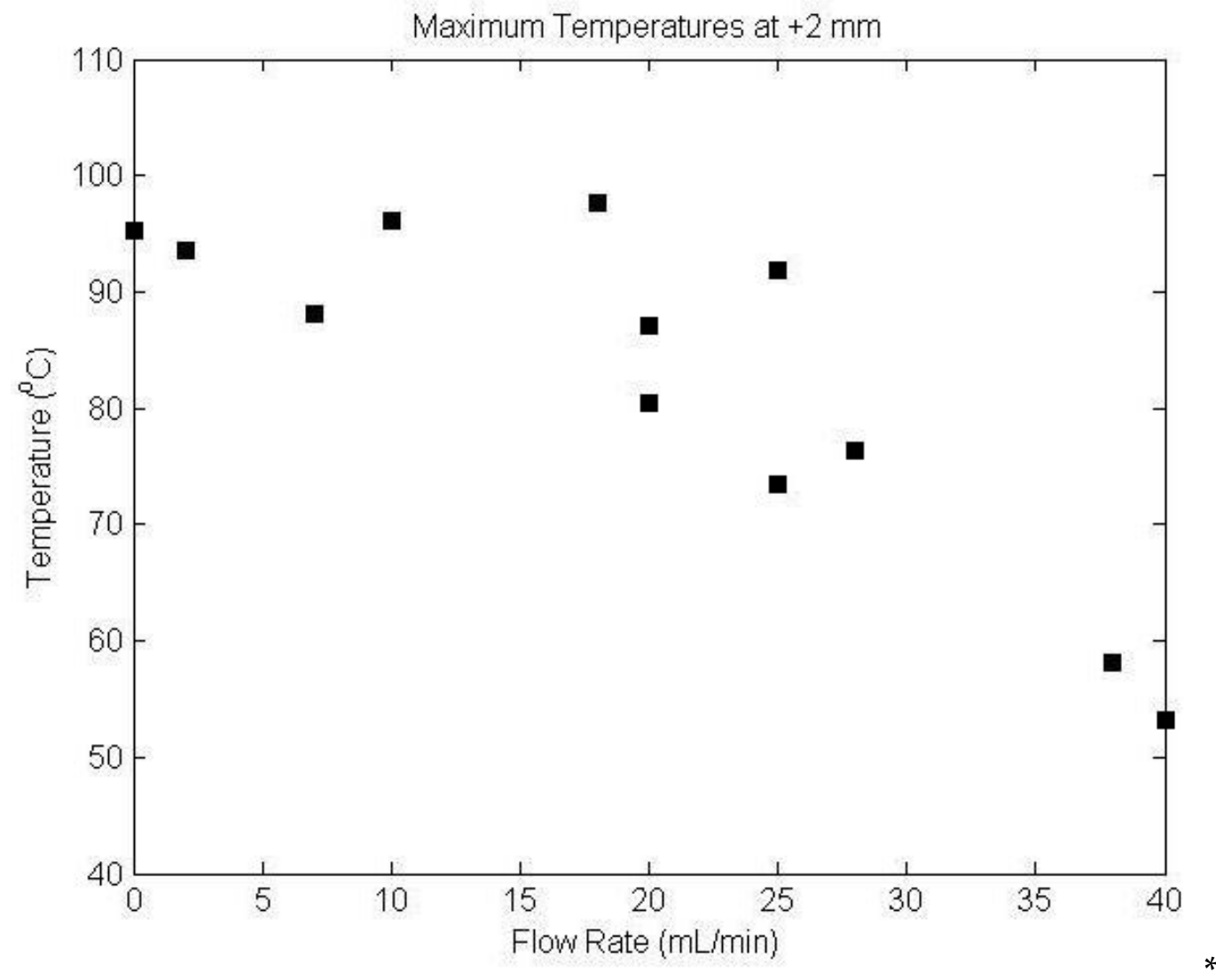

Figure 30. Maximum temperatures at $+2 \mathrm{~mm}$ (side nearest fluid vessel) as a function of flow rate

This plot shows a clear trend of increased temperatures at $+2 \mathrm{~mm}$ as the flow rate drops from $40 \mathrm{~mL} / \mathrm{min}$ to $0 \mathrm{~mL} / \mathrm{min}$ at total vessel occlusion. At 40 $\mathrm{mL} / \mathrm{min}$ the maximum recorded temperature was $53.1^{\circ} \mathrm{C}$, whereas with total vessel occlusion the maximum temperature was recorded as $95.2^{\circ} \mathrm{C}$. This is a significant temperature increase with implications of avoiding undertreated cold spots during HIFU tissue ablation. 
The same trend was not observed on the left side of the HIFU focus at -2 $\mathrm{mm}$, although peak temperatures never went below $64^{\circ} \mathrm{C}$. This fact is important because the lower limit for ablation therapy is approximately $50-55^{\circ} \mathrm{C}$. The comparison of temperatures at $+2 \mathrm{~mm}$ and $-2 \mathrm{~mm}$ as a function of flow rate is shown in Figure 31.

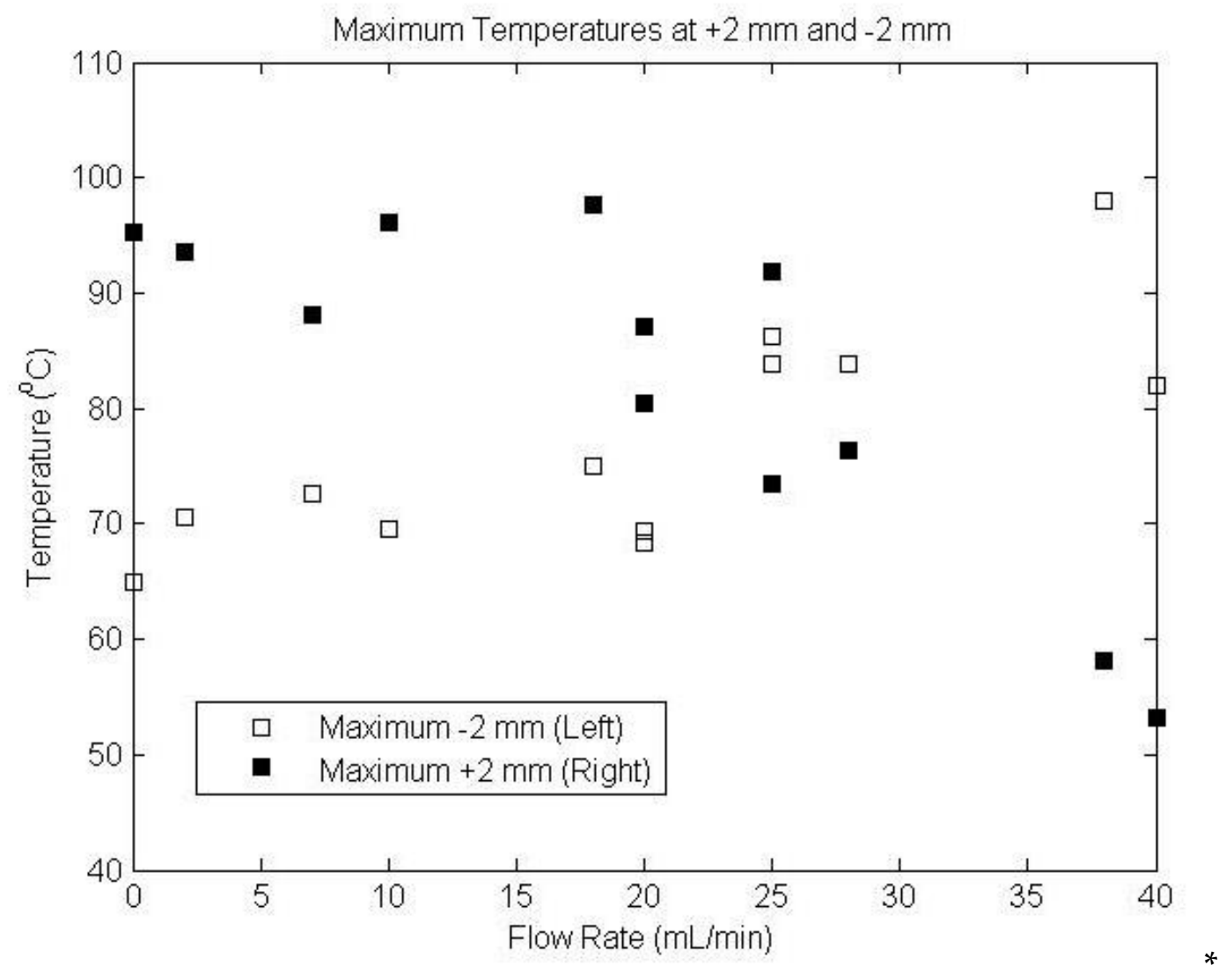

Figure 31. Comparison of maximum temperatures at $+2 \mathrm{~mm}$ and $-2 \mathrm{~mm}$ as a function of flow rate

Counter-intuitively, these data suggest that temperature increases with higher flow rate. The opposite is true on the right side of the HIFU focus, as is 
clearly seen in Figure 31. This may be a result of unexpected movement of the thermocouples on the left side (side farthest from the fluid vessel) as compression was applied. If movement caused the thermocouples to move farther from the HIFU focus, the temperatures would naturally decrease, rather than the expected response of temperatures increasing as flow rate decreases.

The expectation, based on theory of convective heat transfer, is that compression should reduce the asymmetry between the temperatures on the left and right sides of the HIFU focal point. The experimental results show that the asymmetry is substantially reduced when comparing certain levels of compression to the case of zero compression. The data shows that the expected reduction in asymmetry occurs at intermediate levels of compression that bring the flow rate into the range of $28-25 \mathrm{~mL} / \mathrm{min}$. At higher levels of compression, including when vessel occlusion occurs, the asymmetry actually worsens.

Furthermore, we expect that the left-sided temperatures should always exceed those on the right side since convection is present on the right side; however, at flow rates less than or equal to $25 \mathrm{~mL} / \mathrm{min}$, temperatures at $2 \mathrm{~mm}$ on the right side exceed the temperatures at $2 \mathrm{~mm}$ on the left side. The temperatures measured at $2 \mathrm{~mm}$ on the left side also show an overall downward trend as flow rate decreases, which is counter-intuitive to the theory that both left and right sides should increase in temperature as flow rate decreases. While the 
reasons for these unexpected results are not known for certain, I strongly suspect that changes in the tissue-mimicking material integrity caused by repeated compression, as well as possible viscous heating effects at the surfaces of the thermocouples, caused these unexpected results. Solutions for these potential problems are described in the Future Work section (6.2).

\subsection{Simulation Results}

The two-dimensional heat equation was solved using the explicit FD method, incorporating the heat generation calculated by the HIFU beam simulator. As a baseline, the heat equation was solved with no convection first, providing the ideal temperature profile with HIFU heating and pure conduction. The 2-D temperature profile and the 1-D temperature plot (radial vector passing through the center of the HIFU focus) are shown in Figure 32. 


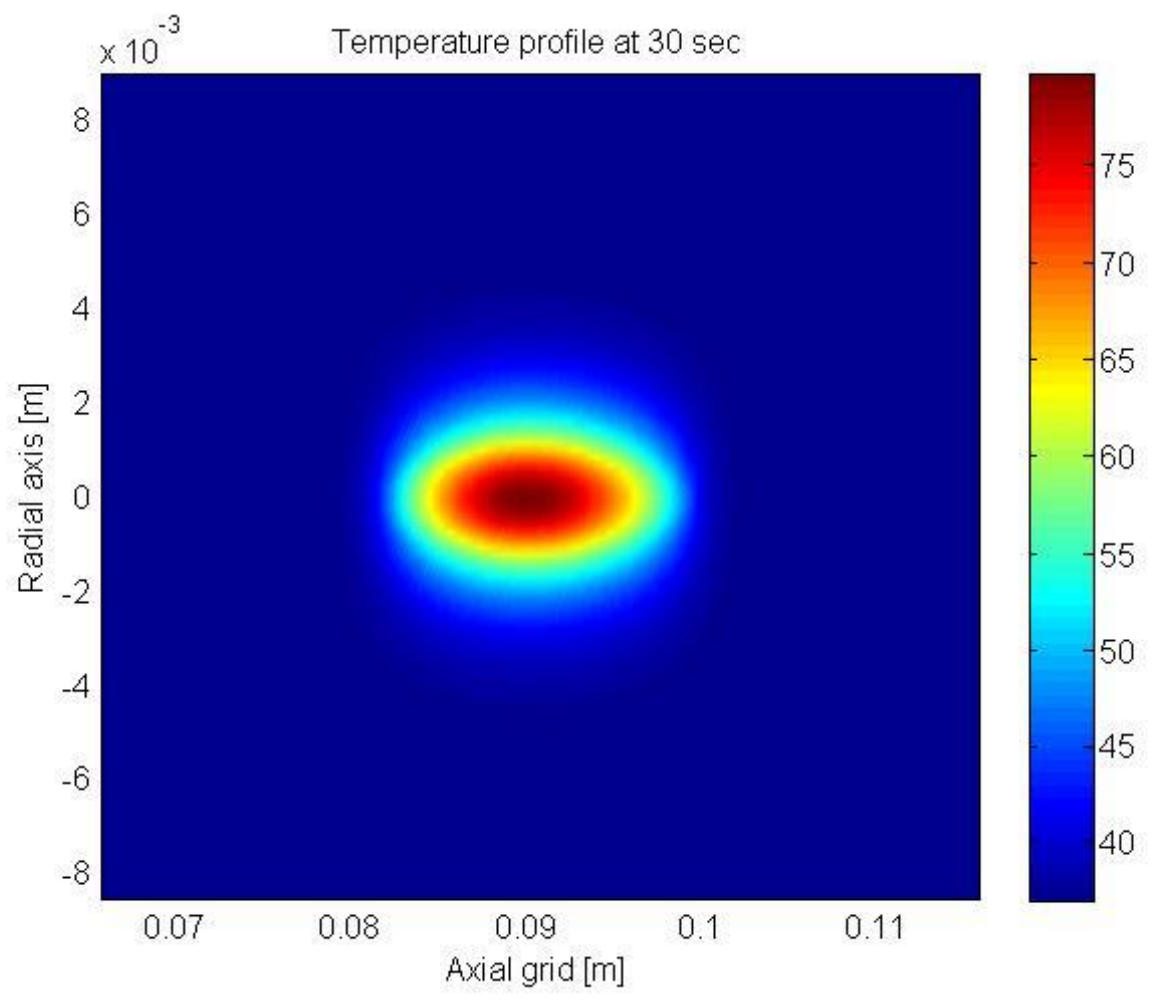

Figure 32 (a) Simulated temperature profile after 30 seconds of heating, no flow

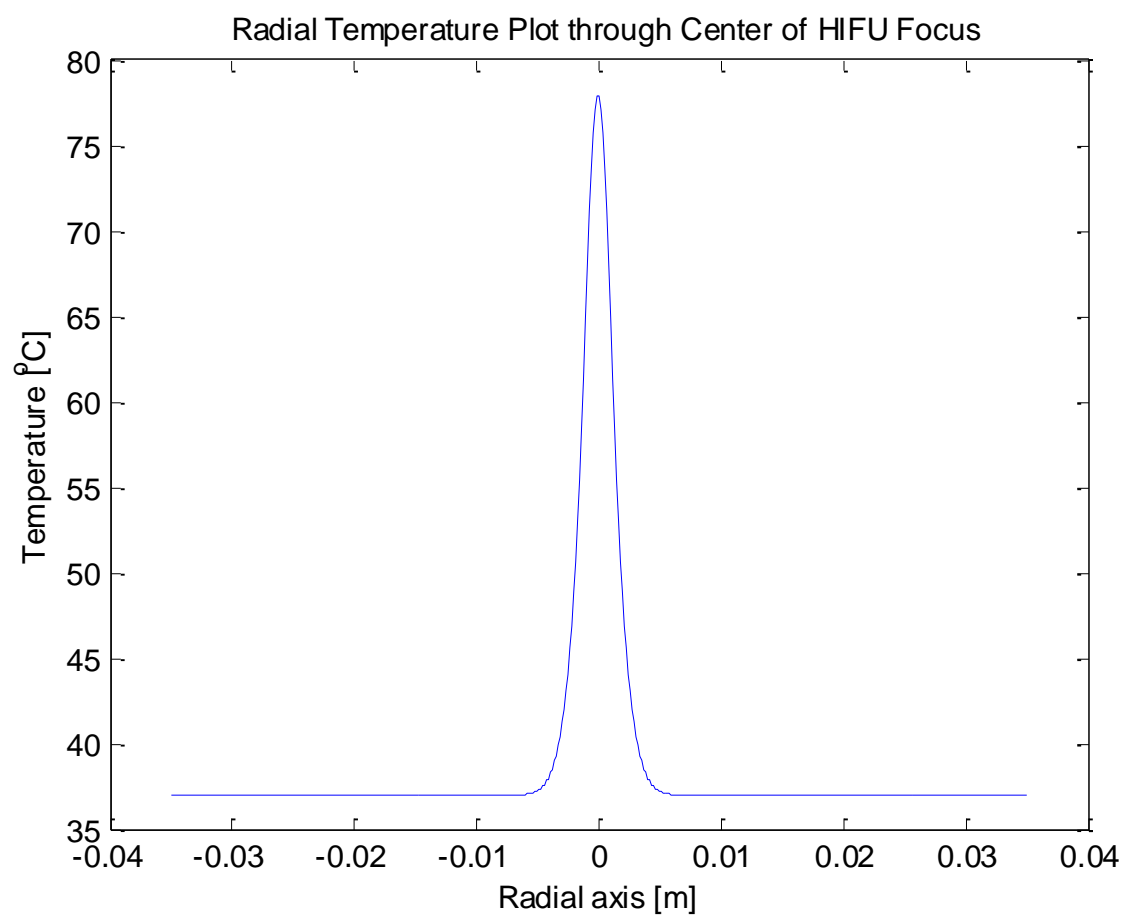

Figure 32 (b) Radial temperature plot after 30 seconds of heating, no flow 
The addition of a $6 \mathrm{~mm}$ vessel, with fluid running through it at $23^{\circ} \mathrm{C}$, adds a convection term to the heat equation. In order to produce temperature profiles comparable to the experimental results, the flow through the vessel was swept through the same range as was recorded during the compression experiments (values shown in Figure 25). The resulting two-dimensional temperature profile and 1-dimensional radial temperature plot for the initial case of $40 \mathrm{~mL} / \mathrm{min}\left(h=114 \mathrm{~W} /\left(\mathrm{m}^{2} \cdot K\right)\right)$ are shown in Figure 33 .

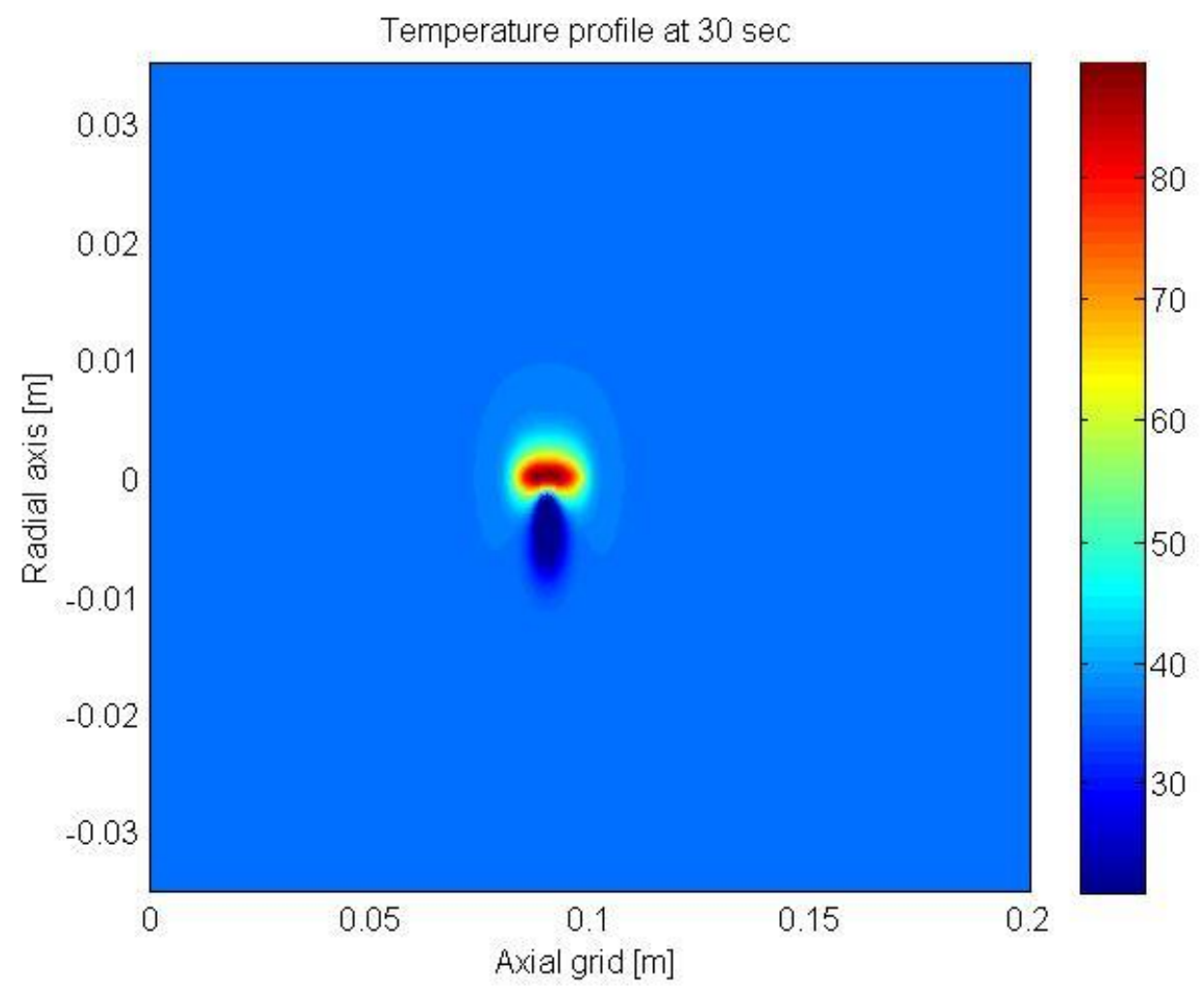

Figure 33 (a) Simulated temperature profile with $40 \mathrm{~mL} / \mathrm{min}$ of flow 


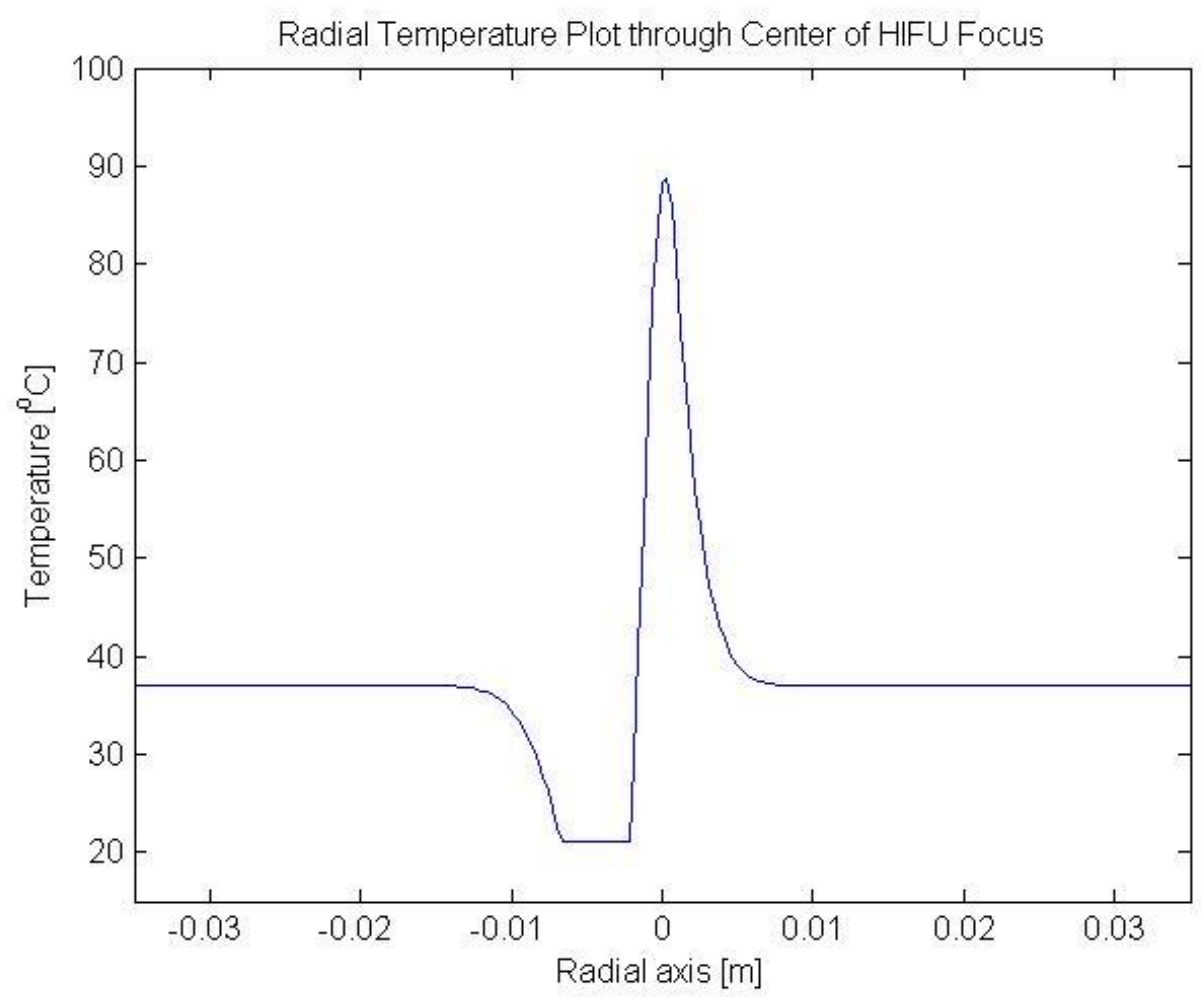

Figure 33 (b) Radial temperature plot with $40 \mathrm{~mL} / \mathrm{min}$ of flow

Based on the experimental results, the flow rates investigated in the simulations were 40,30, 20, 10 and $0 \mathrm{~mL} / \mathrm{min}$. Approximate vessel geometries were assumed for each level of flow investigated in order to compensate for the decrease in vessel cross-sectional area as deformation increased. The temperature profiles calculated for the various flow rates/vessel geometries are shown in Figure 34. 


\section{Flow rate of $40 \mathrm{~mL} / \mathrm{min}$}
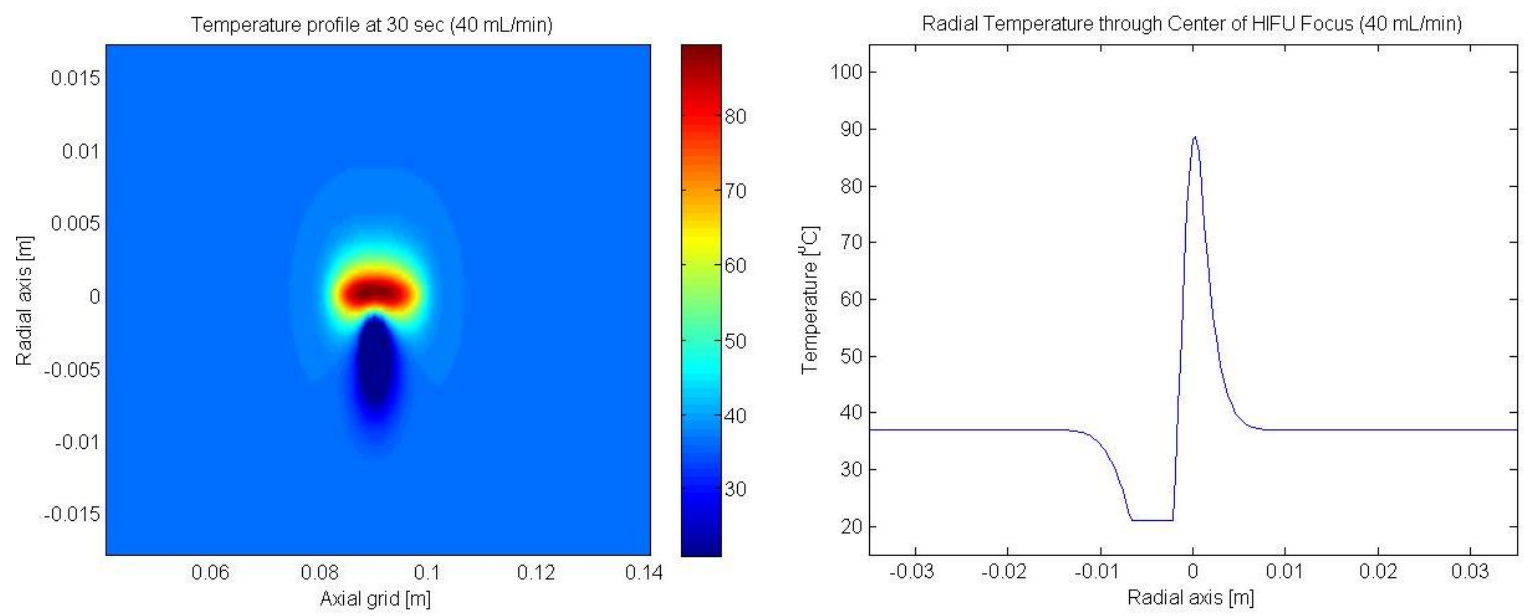

Figure 34 (a) Temperature profile and (b) 1-D radial plot after $30 \mathrm{sec}$ of heating

\section{Flow rate of $30 \mathrm{~mL} / \mathrm{min}$}
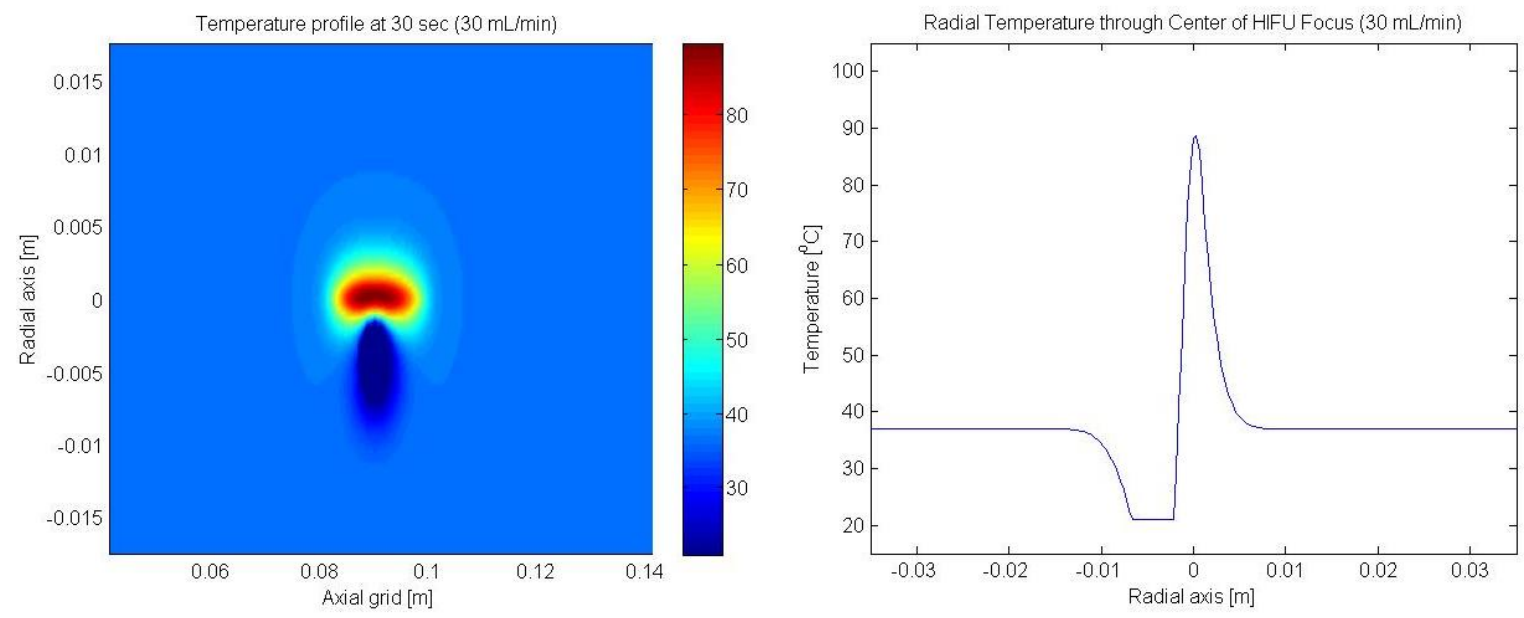

Figure 34 (c) Temperature profile and (d) 1-D radial plot after $30 \mathrm{sec}$ of heating 


\section{Flow rate of $20 \mathrm{~mL} / \mathrm{min}$}
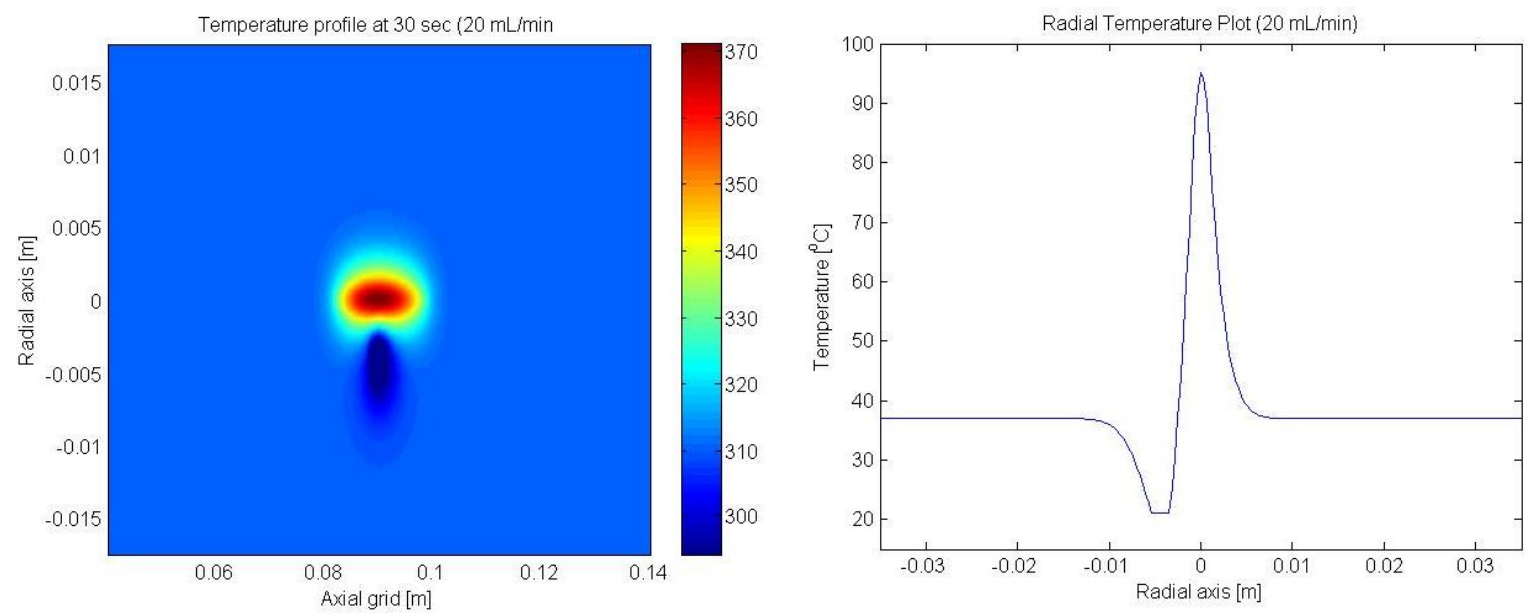

Figure 34 (e) Temperature profile and (f) 1-D radial plot after $30 \mathrm{sec}$ of heating

\section{Flow rate of $10 \mathrm{~mL} / \mathrm{min}$}
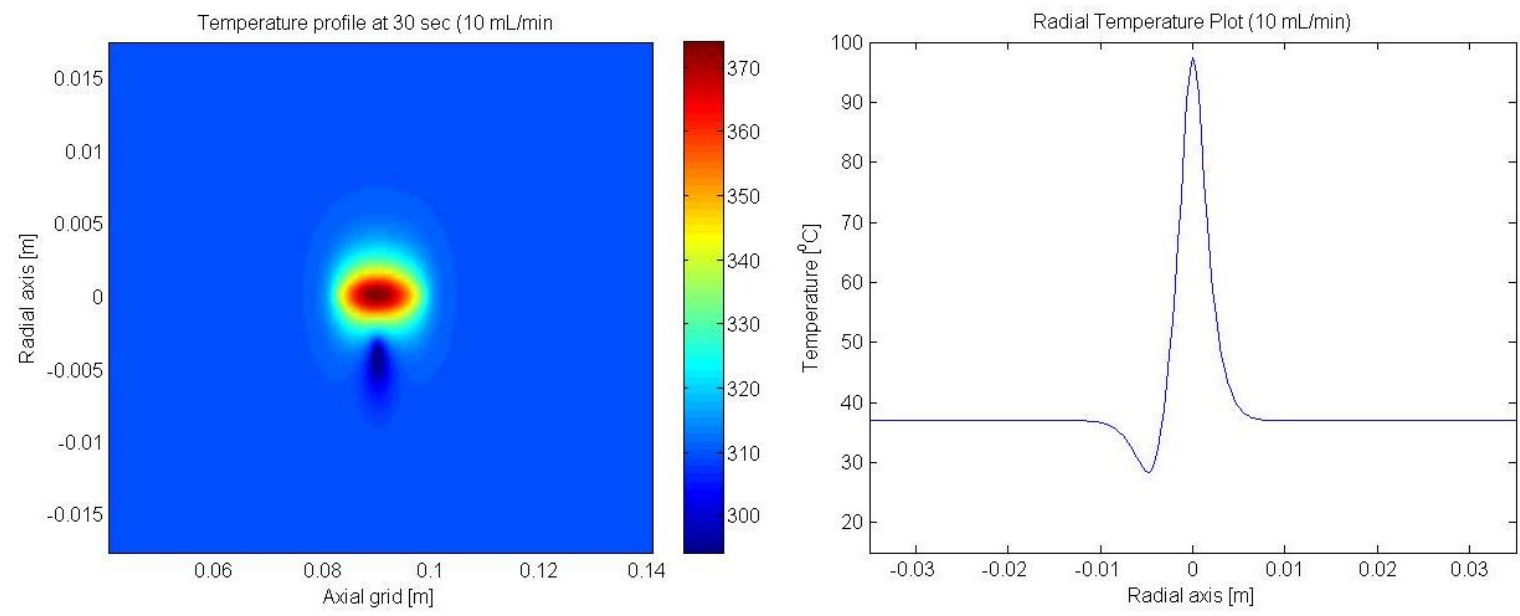

Figure $34(\mathrm{~g})$ Temperature profile and (h) 1-D radial plot after $30 \mathrm{sec}$ of heating 


\section{No flow (total vessel occlusion)}
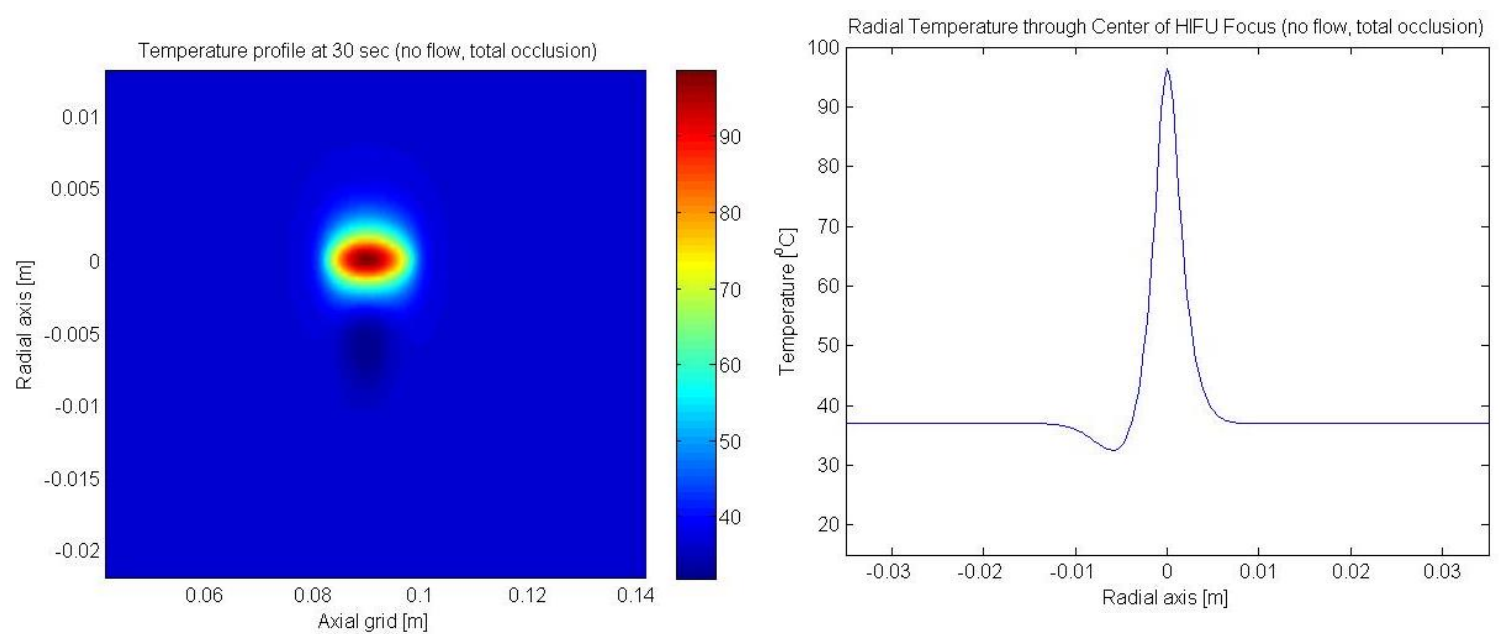

Figure $34(\mathrm{~g})$ Temperature profile and (h) 1-D radial plot after $30 \mathrm{sec}$ of heating

The changes in spatial heating uniformity are difficult to distinguish until the case of total vessel occlusion. By eliminating the convection from the local heat transfer problem, the temperature profile becomes much more uniform, looking essentially the same as the pure conduction solution. The exact values of the calculated uniformity metric for 2, 4 and $6 \mathrm{~mm}$ are shown in Table 5 for the cases of $40 \mathrm{~mL} / \mathrm{min}$ and total vessel occlusion (no flow).

Table 5. Measurement of Simulated Uniformity Metrics

\begin{tabular}{|c|c|}
\hline \multicolumn{2}{|c|}{ Flow rate of $\mathbf{4 0} \mathbf{~} \mathbf{L} / \mathbf{m i n}$} \\
\hline Distance from HIFU focus & Value \\
\hline $\mathbf{2 m m}\left(\boldsymbol{d} \boldsymbol{T}_{\mathbf{2 m m}}=\left|T_{2 m m, L}-T_{2 m m, R}\right|\right)$ & $42.1^{\circ} \mathrm{C}$ \\
\hline $\mathbf{4 m m}\left(\boldsymbol{d} \boldsymbol{T}_{\mathbf{4 m m}}=\left|T_{4 m m, L}-T_{4 m m, R}\right|\right)$ & $21.2^{\circ} \mathrm{C}$ \\
\hline $\mathbf{6 m m}\left(\boldsymbol{d} \boldsymbol{T}_{\mathbf{6 m m}}=\left|T_{6 m m, L}-T_{6 m m, R}\right|\right)$ & $16.9^{\circ} \mathrm{C}$ \\
\hline
\end{tabular}




\begin{tabular}{|c|c|}
\hline \multicolumn{2}{|c|}{ Flow rate of $30 \mathrm{~mL} / \mathrm{min}$} \\
\hline Distance from HIFU focus & Value \\
\hline $\mathbf{2 m \boldsymbol { m }}\left(\boldsymbol{d} \boldsymbol{T}_{\mathbf{2 m m}}=\left|T_{2 m m, L}-T_{2 m m, R}\right|\right)$ & $26^{\circ} \mathrm{C}$ \\
\hline $\mathbf{4 m m}\left(\boldsymbol{d} \boldsymbol{T}_{4 \boldsymbol{m} m}=\left|T_{4 m m, L}-T_{4 m m, R}\right|\right)$ & $21.4{ }^{\circ} \mathrm{C}$ \\
\hline $\mathbf{6 m m}\left(\boldsymbol{d} \boldsymbol{T}_{\mathbf{6 m m}}=\left|T_{6 m m, L}-T_{6 m m, R}\right|\right)$ & $16.9^{\circ} \mathrm{C}$ \\
\hline \multicolumn{2}{|c|}{ Flow rate of $20 \mathrm{~mL} / \mathrm{min}$} \\
\hline Distance from HIFU focus & Value \\
\hline $2 \boldsymbol{m m}\left(\boldsymbol{d} \boldsymbol{T}_{2 m m}=\left|T_{2 m m, L}-T_{2 m m, R}\right|\right)$ & $17.4{ }^{\circ} \mathrm{C}$ \\
\hline $\mathbf{4 m m}\left(\boldsymbol{d} \boldsymbol{T}_{4 \boldsymbol{m} \boldsymbol{m}}=\left|T_{4 m m, L}-T_{4 m m, R}\right|\right)$ & $21.4{ }^{\circ} \mathrm{C}$ \\
\hline $\mathbf{6 m m}\left(\boldsymbol{d} \boldsymbol{T}_{\mathbf{6 m m}}=\left|T_{6 m m, L}-T_{6 m m, R}\right|\right)$ & $16.9^{\circ} \mathrm{C}$ \\
\hline \multicolumn{2}{|c|}{ Flow rate of $10 \mathrm{~mL} / \mathrm{min}$} \\
\hline Distance from HIFU focus & Value \\
\hline $\mathbf{2 m \boldsymbol { m }}\left(\boldsymbol{d} \boldsymbol{T}_{\mathbf{2 m m}}=\left|T_{2 m m, L}-T_{2 m m, R}\right|\right)$ & $5.6^{\circ} \mathrm{C}$ \\
\hline $\mathbf{4 m m}\left(\boldsymbol{d} \boldsymbol{T}_{\mathbf{4 m m}}=\left|T_{4 m m, L}-T_{4 m m, R}\right|\right)$ & $21.5^{\circ} \mathrm{C}$ \\
\hline $\mathbf{6 m m}\left(\boldsymbol{d} \boldsymbol{T}_{\mathbf{6 m m}}=\left|T_{6 m m, L}-T_{6 m m, R}\right|\right)$ & $10.1^{\circ} \mathrm{C}$ \\
\hline \multicolumn{2}{|c|}{ No flow, total occlusion } \\
\hline Distance from HIFU focus & Value \\
\hline $\mathbf{2 m \boldsymbol { m }}\left(\boldsymbol{d} \boldsymbol{T}_{2 \boldsymbol{m m}}=\left|T_{2 m m, L}-T_{2 m m, R}\right|\right)$ & $1.5^{\circ} \mathrm{C}$ \\
\hline $\mathbf{4 m m}\left(\boldsymbol{d} \boldsymbol{T}_{4 \boldsymbol{m m}}=\left|T_{4 m m, L}-T_{4 m m, R}\right|\right)$ & $11.5^{\circ} \mathrm{C}$ \\
\hline $\mathbf{6 m m}\left(\boldsymbol{d} \boldsymbol{T}_{6 \boldsymbol{6 m m}}=\left|T_{6 m m, L}-T_{6 m m, R}\right|\right)$ & $3.3^{\circ} \mathrm{C}$ \\
\hline
\end{tabular}

The numerical results illustrate very clearly the effect that a vessel located near a HIFU focus has during ablation. As compression causes the flow rate to decrease, there is an improvement in spatial heating uniformity at 2, 4 and $6 \mathrm{~mm}$ (from the HIFU focus). The difference between $40 \mathrm{~mL} / \mathrm{min}$ and total occlusion 
shows an obvious improvement in uniformity, but the difference between 40 and $30 \mathrm{~mL} / \mathrm{min}$, for example, is more subtle. Part of the reason for this may be ineffective approximation of the deformed vessel geometry during compression. If the model of the compressed vessel geometry is much larger than in reality, the decrease in convective heat transfer due to compression may not affect the heat transfer enough to be discernible by simulation. A more sophisticated simulation of the solid mechanics at work during tissue compression would likely improve the spatial resolution of the results. 


\section{CHAPTER SIX}

\section{CONCLUSIONS AND FUTURE WORK}

\subsection{Conclusions}

The experimental and simulated findings of this investigation

demonstrate the effect that compression and the corresponding decrease in flow rate through an embedded vessel has on the temperature distribution of tissuemimicking material during HIFU ablation. Based on both the experimental and simulated results, the temperature at $2 \mathrm{~mm}$ on the side closest to the fluid vessel consistently increases with the application of compression and associated flow rate decrease. This is expected because decreased flow rate means less convective heat transfer. The ability to increase the temperatures near a vessel during HIFU heating is important because cold spots can lead to incomplete ablation of cancerous tissues. Experimental data showed that local temperature increases in the range of $40{ }^{\circ} \mathrm{C}$ to $45^{\circ} \mathrm{C}$ could be achieved by applying compression to the outer surface of a tissue-mimicking phantom.

Furthermore, the heating asymmetry that occurs when a HIFU focus is near a fluid vessel can be improved through application of external compression. Experimental data showed left and right $2 \mathrm{~mm}$ temperatures coming within 5.7 ${ }^{\circ} \mathrm{C}$ of transverse symmetrical heating. The ability to improve the heating 
asymmetry of HIFU heating in perfused tissue has the potential to improve tumor ablation planning significantly.

\subsection{Future Work}

The successes and shortcomings of this investigation point to several pathways for future work. The difficulties of designing and testing physical tissue-mimicking phantoms make the use of simulations an attractive approach, but more work needs to be done to validate the model used in this investigation. A simplified tissue-mimicking phantom and corresponding numerical model can be used to validate the simulation approach and justify confidence in the numerical results of this investigation. An ideal model for initial pursuit could feature a small block of tissue-mimicking material, eliminating the effect of an embedded fluid vessel. The physical phantom, including a single embedded thermocouple, could be built and characterized precisely so that the material properties can be inserted into the numerical model directly. By comparing the experimental and simulated results, the validity of the model, assumptions and numerical methods used in this thesis can be assessed.

The assumption of the lesion size created by the $1.13 \mathrm{MHz}$ HIFU transducer used in this investigation can also be evaluated using a polyacrylamide/BSA phantom. This phantom type allows for visualization of 
HIFU lesions in real-time. The lesion size should be measured to make sure that the assumption of the relevance of 2, 4 and $6 \mathrm{~mm}$ temperatures is correct. Another aspect that should be followed up on is the homogeneity and mechanical limits of the tissue-mimicking material used in this investigation. If the material is not appropriate for a study on compression of a HIFU phantom, other tissue-mimicking materials should be considered. The proportions of the ingredients can also be investigated to see if the elasticity and mechanical strength can be improved. The mechanical integrity of the material broke down during compression and did not respond elastically, which likely affected the thermal and acoustical properties.

The other factor that may have affected the temperatures read by the thermocouples is a phenomenon known as viscous heating. Viscous heating occurs when mechanical vibrations cause the tissue-mimicking material to move relative to the metal thermocouple junctions, resulting in increased heating at the probe surface. Previous work has been done to evaluate the degree of viscous heating that occurs during HIFU ablation and methods for temperature compensation were suggested. These methods can be evaluated for the experimental setup described in this investigation in an attempt to identify whether viscous heating is occurring and how to compensate for it if it is present. 


\section{REFERENCES}

1. American Cancer Society (ACS). Breast Cancer Facts E Figures 2011-2012 [Online]. Available:

www.cancer.org/acs/groups/content/@epidemiologysurveilance/documents/doc ument/acspc-030975.pdf [accessed 29 March 2012].

2. P Wust, B. Hildebrandt, G. Sreenivasa, G. Rau, J. Gellermann, H. Riess, R. Felix \& P.M. Schlag. "Hyperthermia in combined treatment of cancer." Oncology, vol. 3, pp. 487-497, 2002.

3. R.B. Roemer. "Engineering Aspects of Hyperthermia Therapy." Annual Review of Biomedical Engineering, vol. 1, pp. 347-376, 1999.

4. C.W. Song, H.J. Park, C.K. Lee \& R. Griffin. “Implications of increased tumor blood flow and oxygenation caused by mild temperature hyperthermia in tumor treatment." Int. J. Hyperthermia,vol. 21(8), pp. 761-767, 2005.

5. B. Hildebrant, P. Wust, O. Ahlers, A. Dieing, G. Sreenivasa, T. Kerner, R. Felix \& H. Riess. "The cellular and molecular basis of hyperthermia." Hematology, vol. 43, pp. 33-56, 2002.

6. F. Wu, G. Haar \& W.R. Chen. "High-intensity focused ultrasound ablation of breast cancer." Expert Review of Anticancer Therapy, vol. 7(6), pp. 823-831, 2007. 
7. J. Kennedy, G.R. ter Haar \& D.R. Cranston. “High intensity focused ultrasound: surgery of the future?" The British Journal of Radiology, vol. 76, pp. 590-599, 2003.

8. National Institute of Child Health and Human Development. Uterine Fibroid [Online]. Available: www.nichd.nih.gov/health/topics/uterine fibroids.cfm [accessed 8 May 2012].

9. C. Freudenrich. How Ultrasound Works [Online]. Available: www.science.howstuffworks.com/ultrasound2.htm [accessed 3 May 2012].

10. T.R. Shrout. "Innovations in Piezoelectric Materials for Ultrasound Transducers," presented at the IEEE International Symposium, Rome, Italy, 2009.

11. Maple Leaf HIFU. History of Ablatherm HIFU [Online]. Available: www.hifu.ca/history-ablatherm-hifu.htm [accessed 25 May 2012].

12. V. Humphrey. "Ultrasound and matter - Physical interactions." Progress in Biophysics and Molecular Biology, vol. 93, pp. 195-211, 2007.

13. S. Mashouf. “An Enhanced Numerical Model to Simulate Nonlinear Continuous Wave Ultrasound Propagation and the Resulting Temperature Response," M.S. thesis, Ryerson University, Toronto, Ontario, Canada, 2009.

14. B. Kaus, lecture slides. "Finite differences: and what about 2D?" Numerische Methoden 1, Jahannes Gutenberg Universitat Mainz (no date).

15. G. Park, S. Lee \& J. Lee, lecture slides. “How to solve PDEs using MATHEMATICA and MATLAB." Dept. of Electronics and Electrical 
Engineering, Pohang University of Science and Technology, Hyoja-dong, South Korea, 2006.

16. L. Ng. "Numerical methods for solving transient heat conduction problems with combined convection and radiation," M.S. thesis (number 3339), San Jose State University, San Jose, CA, USA, 1990.

17. S. Dasgupta, R. Banerjee, P. Hariharan \& M. Myers. “Beam localization in HIFU temperature measurements using thermocouples, with applications to cooling by large blood vessels." Ultrasonics, vol. 51, pp. 171-180, 2011. doi: $10.1016 /$ j.ultras.2010.07.007

18. C. Guiot, E. Madon, D. Allegro, P. Pianta, B. Baiotto \& P. Gabriele. “Perfusion and thermal filed during hyperthermia. Experimental measurements and modeling in recurrent breast cancer." Phys. Med. Biol., vol. 43, pp. 2831-2843, 1998. doi: $10.1088 / 0031-9155 / 43 / 10 / 012$

19. J. Huang, R. Holt, R. Cleveland \& R. Roy. “Experimental validation of a tractable numerical model for focused ultrasound heating in flow-through tissue phantoms." J. Acoustic Soc. Am., vol. 116, issue 4, pp. 2451-2458, 2004.

20. M. Chen \& K. Holmes. "Microvascular contributions in tissue heat transfer." Annals of the New York Academy of Sciences, vol. 335, issue 1, pp. 137-150, 1980. doi: 10.111/j.1749-6632.1980.tb50742.x 
21. PRLog. Cancer Therapy Market to Cross US \$78 Billion by 2012 [Online]. Available: www.prlog.org/10207574-cancer-therapy-market-to-cross-us-78-billion-by-2012 [Accessed 8 March 2013].

22. MedTech Insight. Thermal Ablation Devices Market Report \#A562 [Online]. Available: www.medtechinsight.com/ReportA562.html [Accessed 4 October 2012].

23. K. Okita, K. Ono \& Y. Matsumoto. “Numerical simulation of tissue ablation in high-intensity focused ultrasound therapy with array transducers." International Journal for Numerical Methods in Fluids, vol. 64, issues 10-12, pp. 1395-1411, December 2010. doi: 10.1002/fld.2467

24. H. Pennes. "Analysis of Tissue and Arterial Blood Temperatures in the Resting Human Forearm." Journal of Applied Physiology, vol. 85, pp. 5-34, 1998.

25. R. Rawnsley, R. Roemer \& A. Dutton. “The simulation of discrete vessel effects in experimental hyperthermia." ASME Journal of Biomechanical Engineering, vol. 116, issue 3, pp. 256-262, 1994. doi: 10.1115/1.2895728

26. A. Narashimhan, lecture notes. "Notes on the Chen-Homes Bio-heat Transfer Model." Indian Institute of Technology Madras, Chennai, India (no date).

27. H. Arkin, X. Xu \& K. Holmes. “Recent Developments in Modeling Heat Transfer In Blood Perfused Tissues." IEEE Transactions on Biomedical Engineering, vol. 41, issue 2, pp. 97-107, 1994. doi: 10.1109/10.284920 
28. I. dos Santos, D. Haemmerich, C. Pinheiro \& A. da Rocha. “Effect of variable heat transfer coefficient on tissue temperature next to a large vessel during radiofrequency tumor ablation." Biomedical Engineering Online, vol. 7, pp. 21, 2008. doi: 10.1186/1475-925X-7-21

29. L. Consiglieri, I. dos Santos \& D. Haemmerich. "Theoretical analysis of the heat convection coefficient in large vessels and the significance for thermal ablative therapies." Phys. Med. Biol., vol. 48, pp. 4125-4134, 2003.

30. O. Hassan, N. Hassan \& Y. Kadah. “Modeling of Ultrasound Hyperthermia Treatment of Breast Tumors," presented at $26^{\text {th }}$ National Radio Science Conference, New Cairo, Egypt, 2009.

31. J. Soneson. HIFU Simulation v1.2 User's Manual [Online]. Available: www.fda.gov/AboutFDA/CentersOffices/OfficeofMedicalProductsandTobacco/C DRH/CDRHOffices/ucm301529.htm [Accessed October 2012, published 2011].

32. Y. Lee. "Numerical Solution of the KZK Equation for Pulsed Finite Amplitude Sounds Beams in Thermoviscous Fluids," Ph.D. dissertation, University of Texas at Austin, Texas, 1993.

33. R. King, Y. Liu, S. Maruvada, B. Herman, K. Wear \& G. Harris. “Development and Characterization of a Tissue Mimicking Material for High Intensity Focused Ultrasound." IEEE Transactions on Ultrasonics, Ferroelectrics and Frequency Control, vol. 58, issue 7, pp. 1397-1405, 2011. 
34. C. Lafon, P. Kaczkowski, S. Vaezy \& M. Noble. Development and Characterization of an Innovative Synthetic Tissue-Mimicking Material for High Intensity Focused Ultrasound (HIFU) Exposures, presented at IEEE Ultrasonics Symposium, pp. 1295-1298, 2001.

35. H. Huang, C. Liauh, T. Shih, T. Horng \& W. Lin. "Significance of blood vessels in optimization of absorbed power and temperature distributions during hyperthermia." International Journal of Heat and Mass Transfer, vol. 53, pp. 5651$5662,2010$.

36. B. Jarosz. Effects of Discrete Blood Vessels in Ultrasound Interstitial Heating from FEA Modeling, presented at EMBS International Conference, Chicago, IL, 2000.

37. E. Sassaroli, K. Li \& B. O'Neill. Modeling of the Impact of Blood Vessel Flow on the Temperature Distribution during Focused Ultrasound Exposure. Presented at the Proceedings of COMSOL Conference, Boston, MA, 2010.

38. I. Hein \& W. O’Brien. “A Flexible Blood Flow Phantom Capable of Independently Producing Constant and Pulsatile Flow with a Predictable Spatial Flow Profile for Ultrasound Flow Measurement Validations." IEEE Transactions on Biomedical Engineering, vol. 39, issue 11, pp. 1111-1122, 1992.

39. T. Poepping, H. Nikolov, M. Thorne \& D. Holdsworth. “A Thin-Walled Carotid Vessel Phantom for Doppler Ultrasound Flow Studies." Ultrasound in Medicine E Biology, vol. 30, issue 8, pp. 1067-1078, 2004. 
40. Sonic Concepts. Test Report: Model H-101-G S/N-129 for San Jose State University. Correspondance [received 18 October 2012].

41. Hayden Kirk. Hayden Kirk RGS04KR-M43-0100-08 data sheet [Online]. Available: http://www.haydonkerk.com/?TabId=311 [accessed 18 April 2013].

42. M. Heydari \& M. Jahed. Prediction of Temperature Distribution and Volume of Lesion During HIFU Therapy, presented at the $6^{\text {th }}$ International Conference on Information Technology, 2009.

43. A. Kotte, G. van Leeuwen, J. de Bree, J. van der Koijk, H. Crezee \& J. Lagendijk. "A description of discrete vessel segments in thermal modeling of tissues." Phys. Med. Biol., vol. 41, pp. 865-884, 1996.

44. L. Jiang, W. Zhan \& M. Loew. “Modeling static and dynamic thermography of the human breast under elastic deformation." Phys. Med. Biol., vol. 56, pp. 187202, 2011. doi: 10.1088/0031-9155/56/1/012.

45. T. Shih, H. Liu \& A. Horng. "Cooling effect of thermally significant blood vessels in perfused tumor tissue during thermal therapy." International Communications in Heat and Mass Transfer, vol. 33, pp. 135-141, 2006. http://dx.doi.org/10.1016/j.ijheatmasstransfer.2010.08.017 


\section{APPENDIX A HIFU SIMULATION MATLAB SCRIPT}

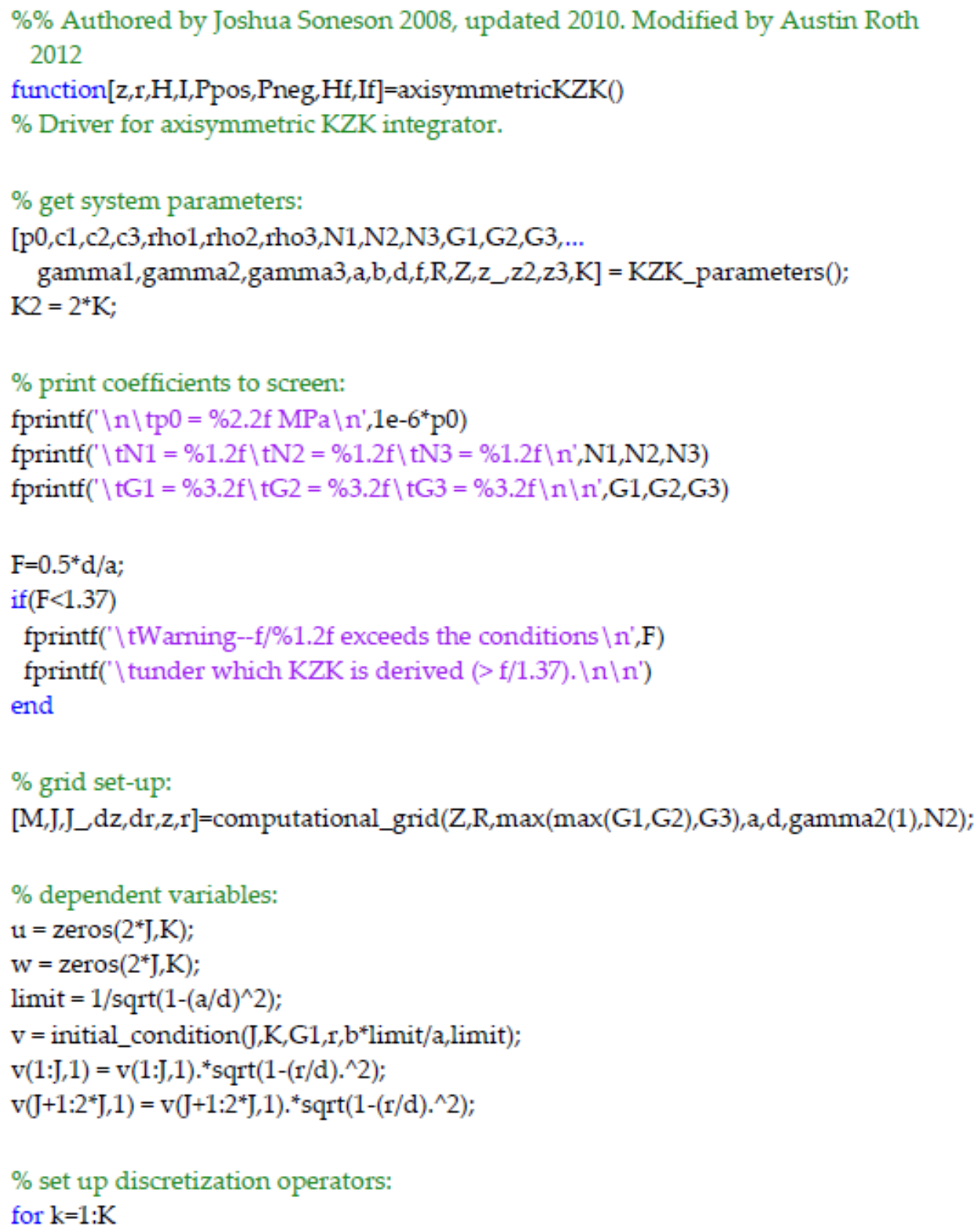




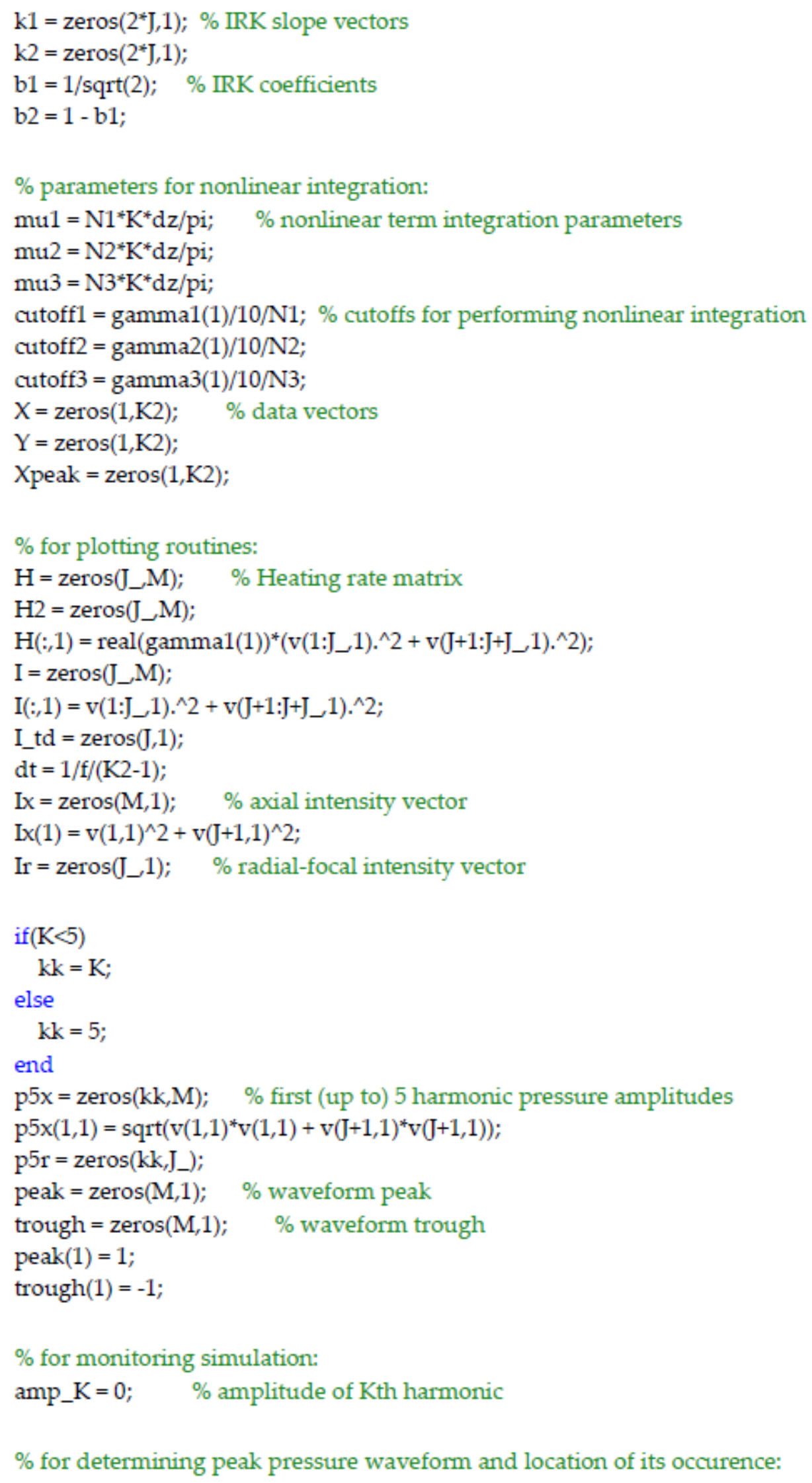




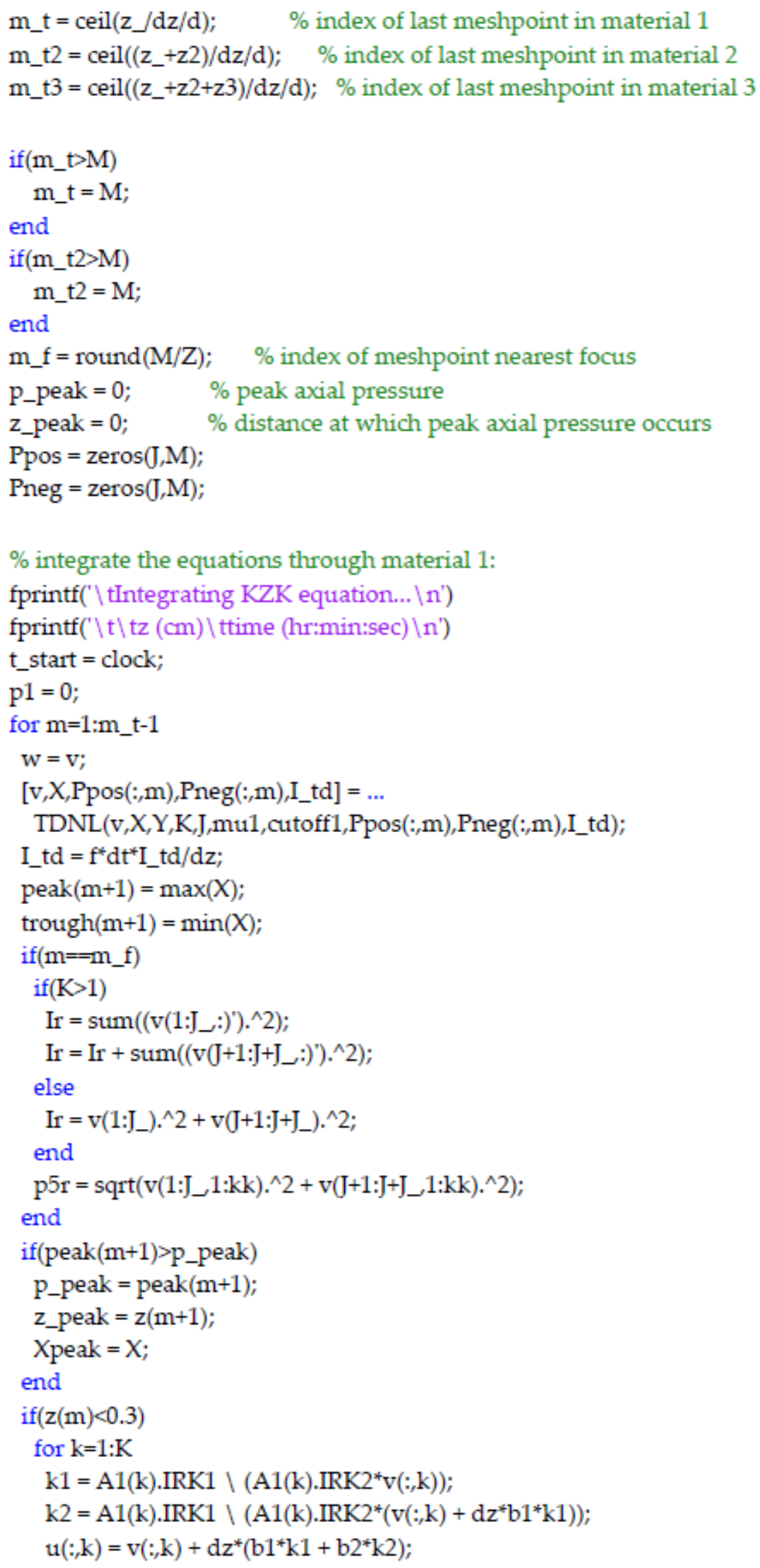




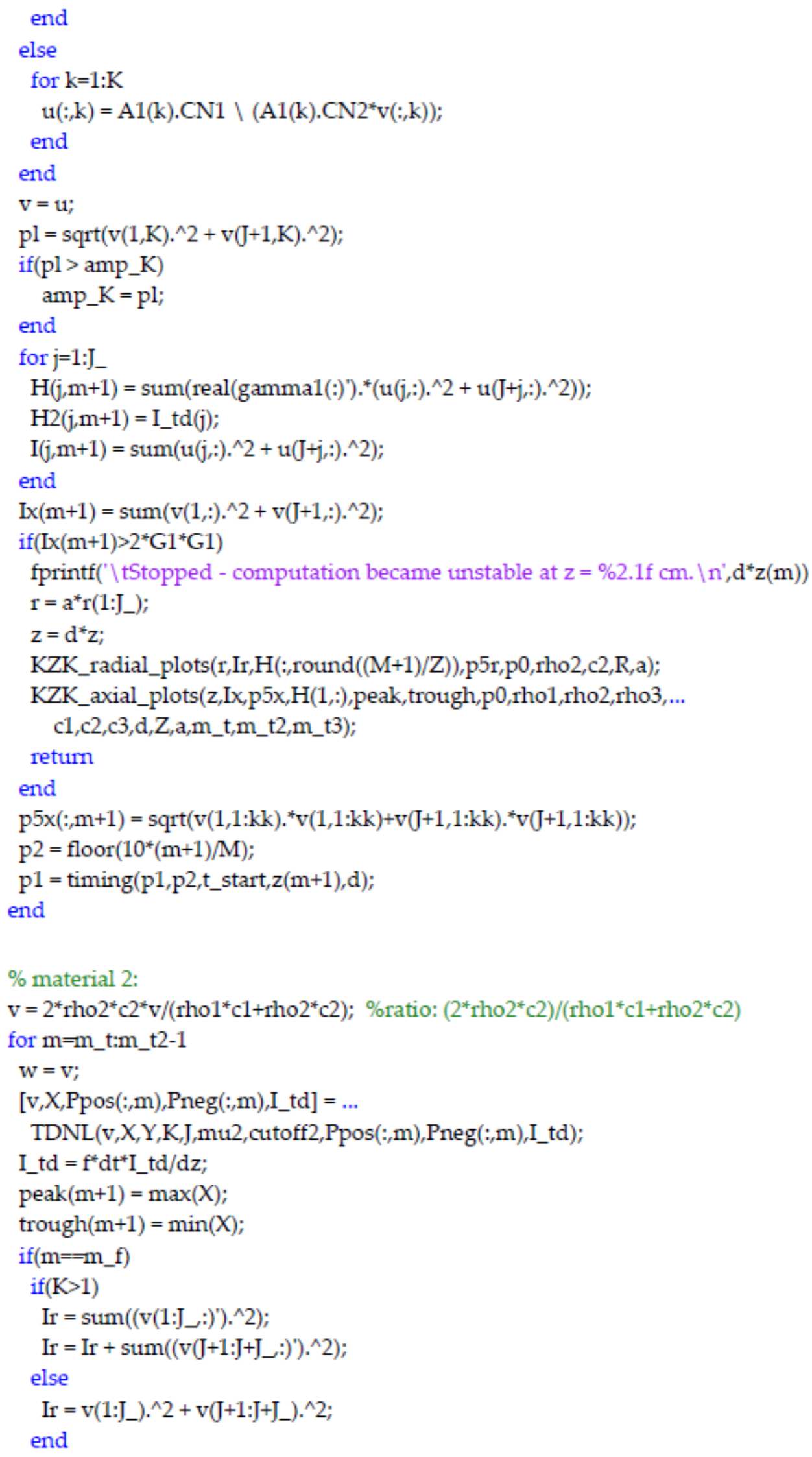




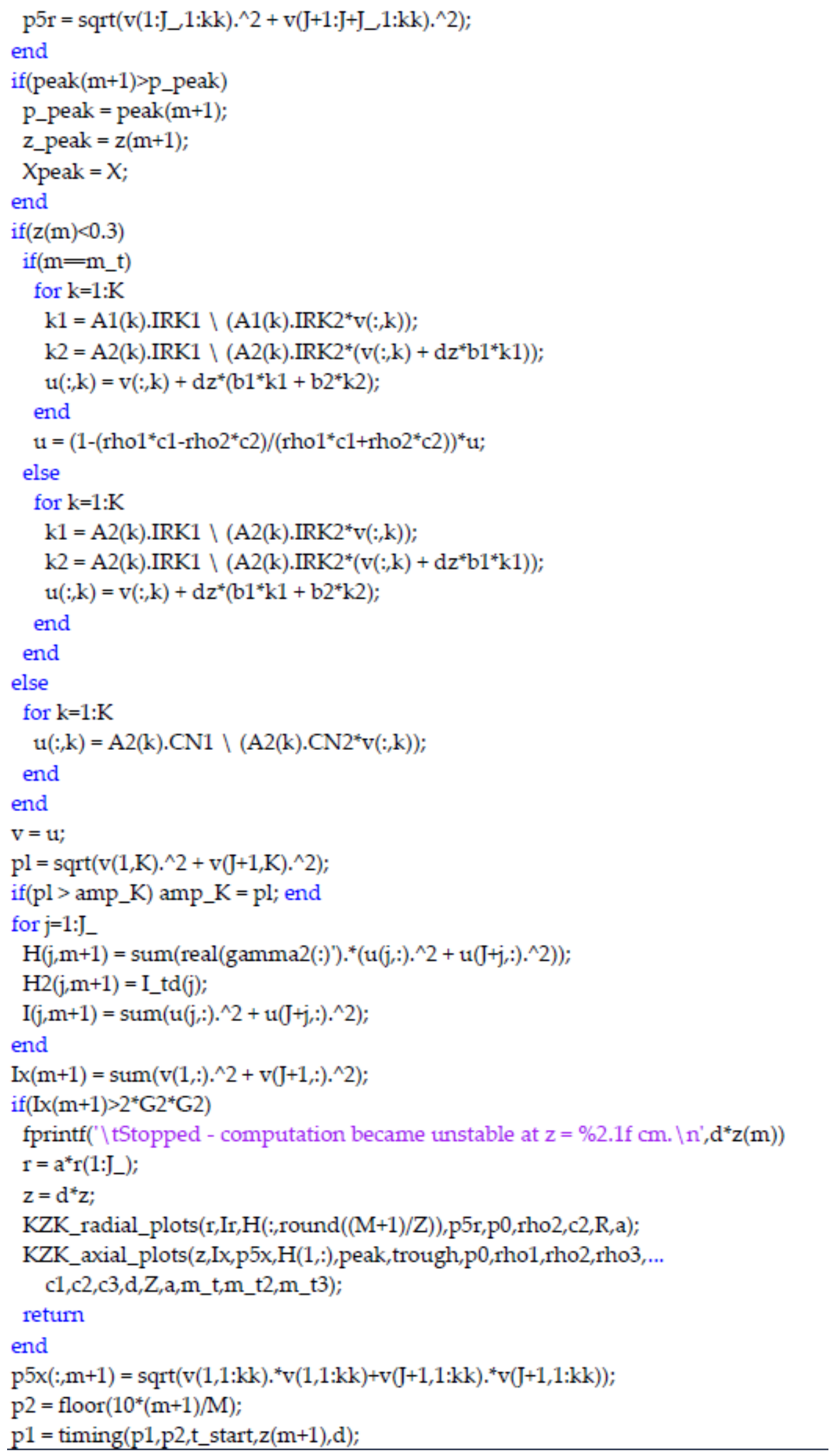




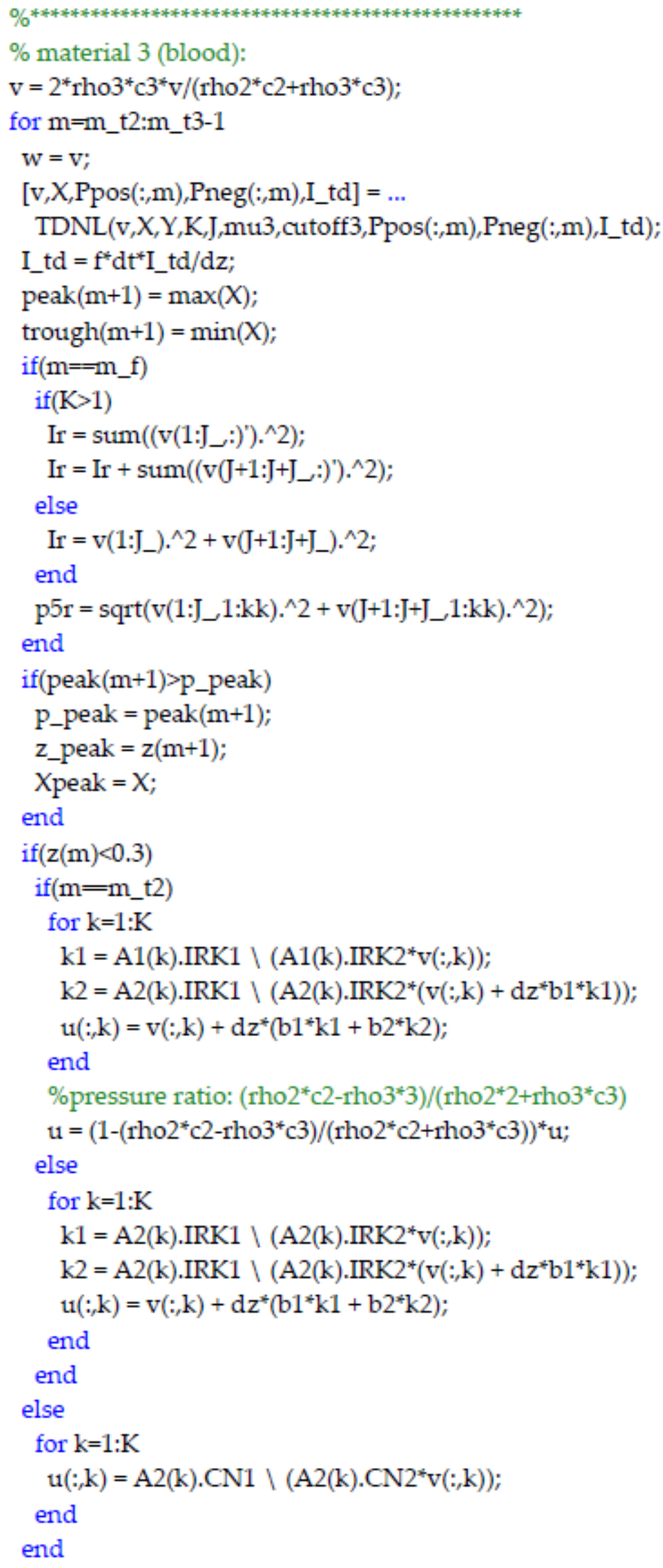




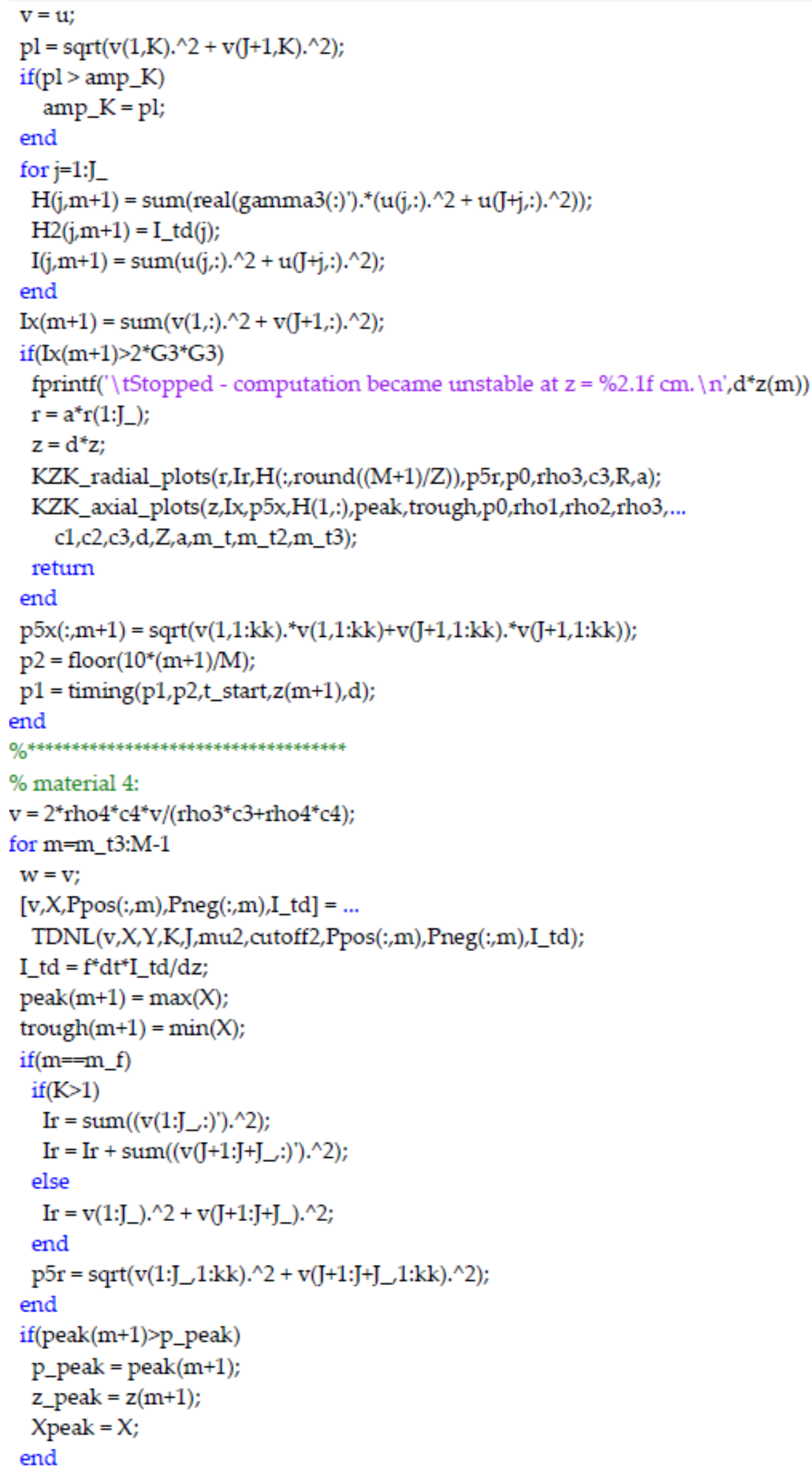




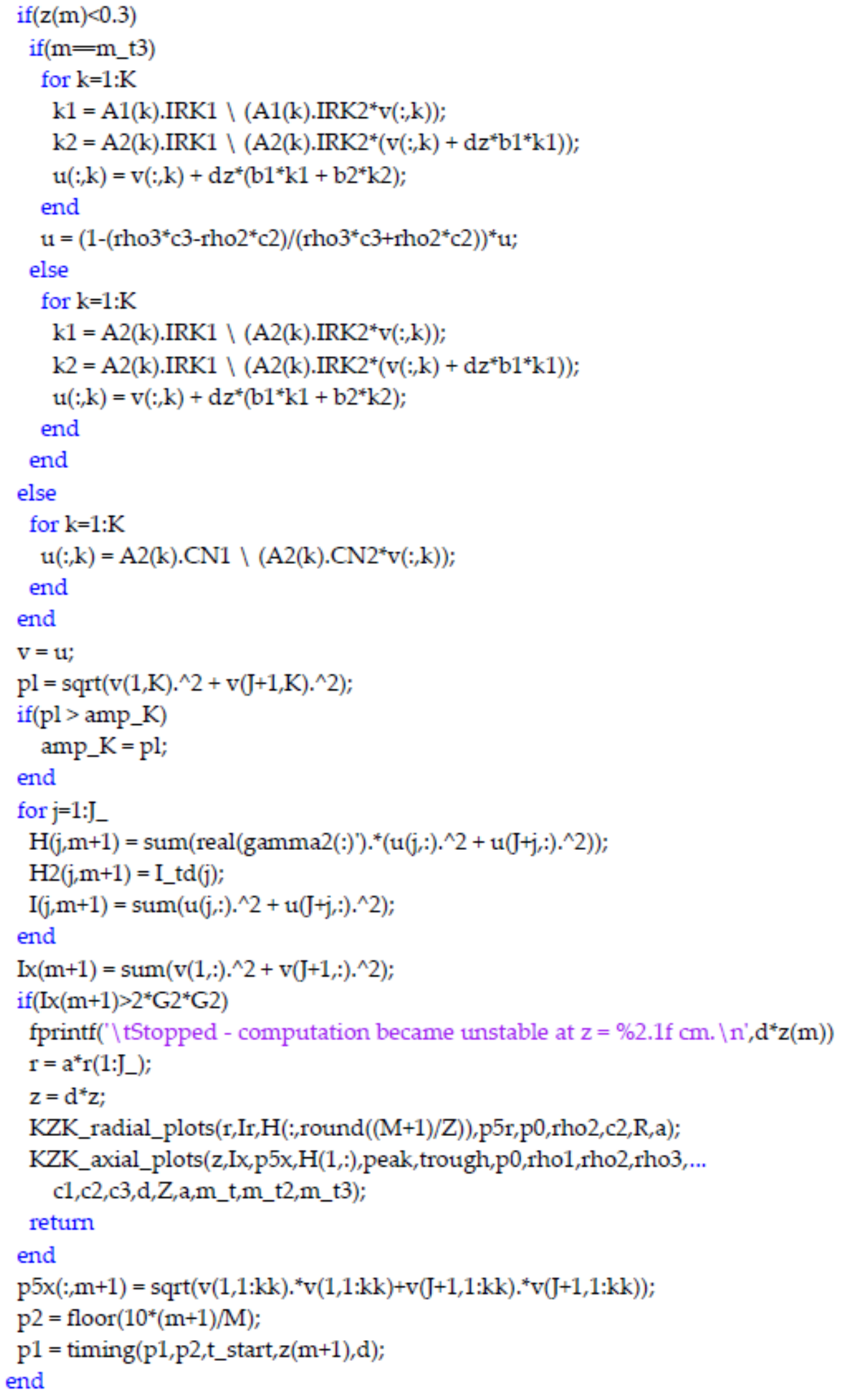


$\mathrm{H}\left(:, 1: \mathrm{m} \_\mathrm{t}\right)=1 \mathrm{e}-4^{*} \mathrm{p}^{*} \mathrm{p} 0 * \mathrm{H}\left(:, 1: \mathrm{m} \_\mathrm{t}\right) / \mathrm{rho} 1 / \mathrm{c} 1 / \mathrm{d} ; \%$ dimensionalize $\mathrm{H}$

$\mathrm{H}\left(:, \mathrm{m}_{-} \mathrm{t}+1: \mathrm{m}_{-} \mathrm{t} 2\right)=1 \mathrm{e}-4^{*} \mathrm{p} 0^{*} \mathrm{p} 0 * \mathrm{H}\left(:, \mathrm{m} \_\mathrm{t}+1: \mathrm{m} \_\mathrm{t} 2\right) / \mathrm{rho} 2 / \mathrm{c} 2 / \mathrm{d}$;

$\mathrm{H}\left(:, \mathrm{m}_{-} \mathrm{t} 2+1: \mathrm{m}_{-} \mathrm{t} 3\right)=1 \mathrm{e}-4^{*} \mathrm{p} 0^{*} \mathrm{p} 0 * \mathrm{H}\left(:, \mathrm{m} \_\mathrm{t} 2+1: \mathrm{m}_{-} \mathrm{t} 3\right) / \mathrm{rho} 3 / \mathrm{c} 3 / \mathrm{d}$;

$\mathrm{H}\left(:, \mathrm{m} \_\mathrm{t} 3+1: \mathrm{M}\right)=1 \mathrm{e}-4^{*} \mathrm{p} 0^{*} \mathrm{p} 0^{*} \mathrm{H}\left(:, \mathrm{m} \_\mathrm{t} 3+1: \mathrm{M}\right) / \mathrm{rho} 2 / \mathrm{c} 2 / \mathrm{d}$;

$\mathrm{H} 2\left(:, 1: \mathrm{m} \_\mathrm{t}\right)=1 \mathrm{e}-4^{*} 0.5^{*} \mathrm{p} 0 * \mathrm{p} 0 * \mathrm{H} 2\left(:, 1: \mathrm{m} \_\mathrm{t}\right) / \mathrm{rho} 1 / \mathrm{c} 1 / \mathrm{d} ; \%$ dimensionalize $\mathrm{H}$

$\mathrm{H} 2\left(:, \mathrm{m} \_\mathrm{t}+1: \mathrm{m} \_\mathrm{t} 2\right)=1 \mathrm{e}-4^{*} 0.5^{*} \mathrm{p} 0^{*} \mathrm{p} 0 * \mathrm{H} 2\left(:, \mathrm{m} \_\mathrm{t}+1: \mathrm{m} \_\mathrm{t} 2\right) / \mathrm{rho} 2 / \mathrm{c} 2 / \mathrm{d}$;

$\mathrm{H} 2\left(:, \mathrm{m} \_\mathrm{t} 2+1: \mathrm{m} \_\mathrm{t} 3\right)=1 \mathrm{e}-4^{*} 0.5^{*} \mathrm{p} 0 * \mathrm{p} 0^{*} \mathrm{H} 2\left(:, \mathrm{m} \_\mathrm{t} 2+1: \mathrm{m} \_\mathrm{t} 3\right) / \mathrm{rho} 3 / \mathrm{c} 3 / \mathrm{d}$;

$\mathrm{H} 2\left(:, \mathrm{m} \_\mathrm{t} 3+1: \mathrm{M}\right)=1 \mathrm{e}-4^{*} 0.5^{*} \mathrm{p} 0 * \mathrm{p}^{*} \mathrm{H} 2\left(:, \mathrm{m} \_\mathrm{t} 3+1: \mathrm{M}\right) / \mathrm{rho} 2 / \mathrm{c} 2 / \mathrm{d}$;

$\mathrm{H}=\operatorname{real}(\mathrm{H}+\mathrm{H} 2)$;

$\mathrm{I}\left(:, 1: \mathrm{m} \_\mathrm{t}\right)=1 \mathrm{e}-4^{*} 0.5^{*} \mathrm{p} 0 * \mathrm{p} 0 * \mathrm{I}\left(:, 1: \mathrm{m} \_\mathrm{t}\right) / \mathrm{rho} 1 / \mathrm{c} 1 ; \%$ dimensionalize I

$\mathrm{I}\left(:, \mathrm{m} \_\mathrm{t}+1: \mathrm{m} \_\mathrm{t} 2\right)=1 \mathrm{e}-4^{*} 0.5^{*} \mathrm{p} 0 * \mathrm{p} 0^{*} \mathrm{I}\left(:, \mathrm{m} \_\mathrm{t}+1: \mathrm{m} \_\mathrm{t} 2\right) / \mathrm{rho} 2 / \mathrm{c} 2 ;$

$\mathrm{I}\left(:, \mathrm{m} \_\mathrm{t} 2+1: \mathrm{m} \_\mathrm{t} 3\right)=1 \mathrm{e}-4^{*} 0.5^{*} \mathrm{p} 0 * \mathrm{p} 0 * \mathrm{I}\left(:, \mathrm{m} \_\mathrm{t} 2+1: \mathrm{m} \_\mathrm{t} 3\right) / \mathrm{rho} 3 / \mathrm{c} 3$;

$\mathrm{I}\left(:, \mathrm{m} \_\mathrm{t} 3+1: \mathrm{M}\right)=1 \mathrm{e}-4^{*} 0.5^{*} \mathrm{p} 0^{*} \mathrm{p} 0 * \mathrm{I}\left(:, \mathrm{m} \_\mathrm{t} 3+1: \mathrm{M}\right) / \mathrm{rho} 2 / \mathrm{c} 2$;

$\operatorname{fprintf}\left(\backslash \operatorname{tmax}\left(\left|\mathrm{p} \_\mathrm{K}\right| / \mathrm{p} 0\right)=\% \mathrm{e} \backslash \mathrm{n}^{\prime}, \mathrm{amp} \_\mathrm{K}\right)$

$\mathrm{r}=\mathrm{a}^{*} \mathrm{r}\left(1: \mathrm{J}_{-}\right) ; \%$ rescale $\mathrm{r}$ and chop so that PML region is excluded in plots

$\mathrm{z}=\mathrm{d}^{*} \mathrm{z} ; \%$ rescale $\mathrm{z}$

Ppos = Ppos(1:J_: :;

Pneg = Pneg(1:J_:);

$\%$ call plotting routines:

if $(\mathrm{K}>1)$

plot_waveform (p0,f,Xpeak, $\mathrm{d}^{*} \mathrm{z}$ _peak,K2);

end

$\mathrm{Hf}=$ zeros $(2 *$ length( $\mathrm{r})-1$, length $(\mathrm{z}))$;

If $=$ zeros $\left(2^{*}\right.$ length $(r)-1$,length $\left.(z)\right)$;

$\mathrm{Hf}\left(\right.$ length $(\mathrm{r}): 2^{*}$ length $\left.(\mathrm{r})-1, \mathrm{i}\right)=\mathrm{H}$;

If(length $(\mathrm{r}): 2 *$ length $(\mathrm{r})-1, \mathrm{:})=\mathrm{I}$;

Hflip=flipdim $(\mathrm{H}, 1)$;

Iflip=flipdim(I,1);

Hmod=Hflip(2:length(r),:);

Imod=Iflip(2:length(r),:; ;

Hf(1:length(r)-1,: $)=$ Hmod;

If $(1:$ length $(\mathrm{r})-1, \mathrm{:})=\mathrm{Imod}$;

figure(1), image(Hf), title('H'), colorbar;

figure(2), image(If), title('I'), colorbar; 


\section{APPENDIX B TEMPERATURE PLOTTING MATLAB SCRIPT}

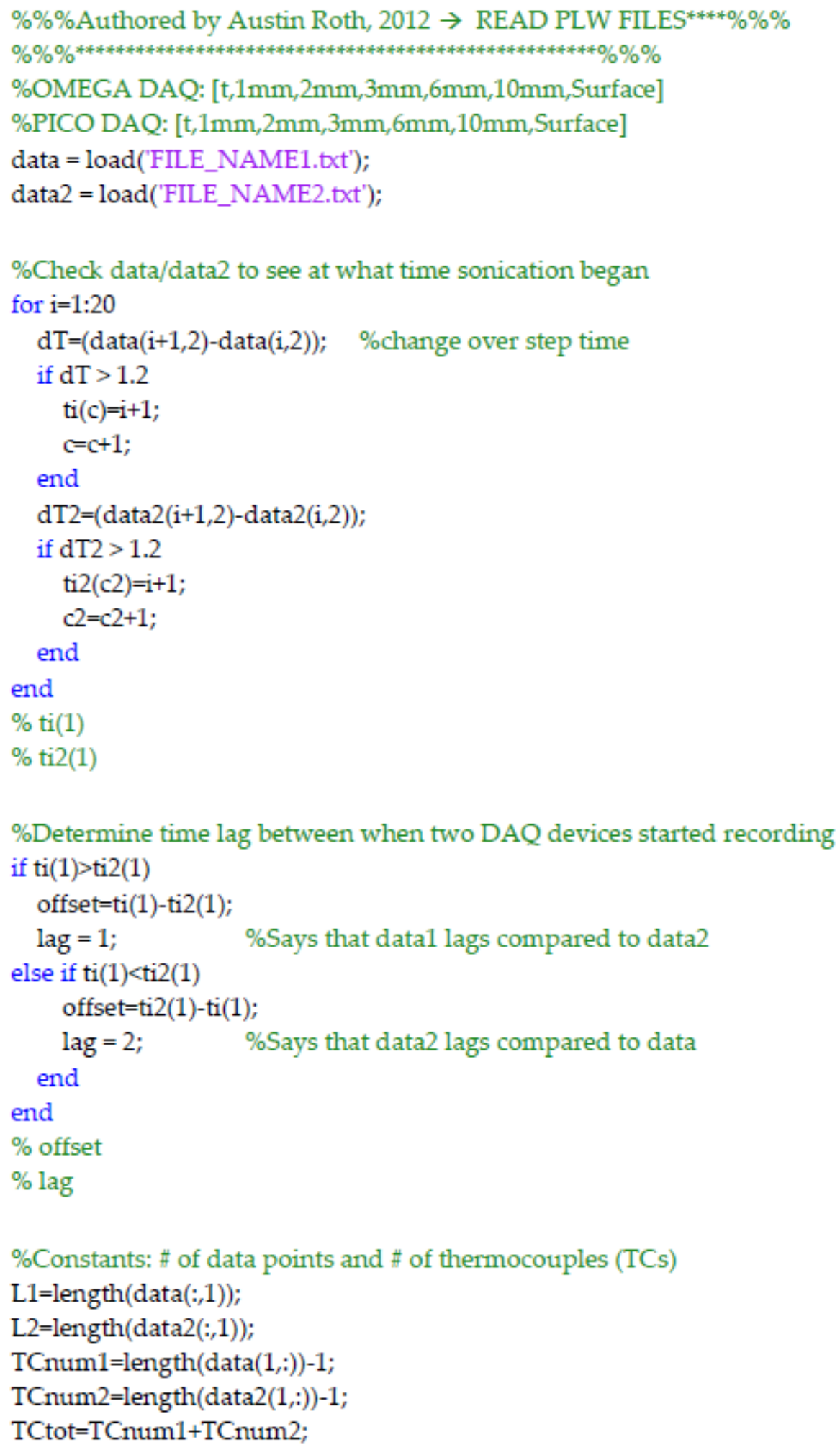




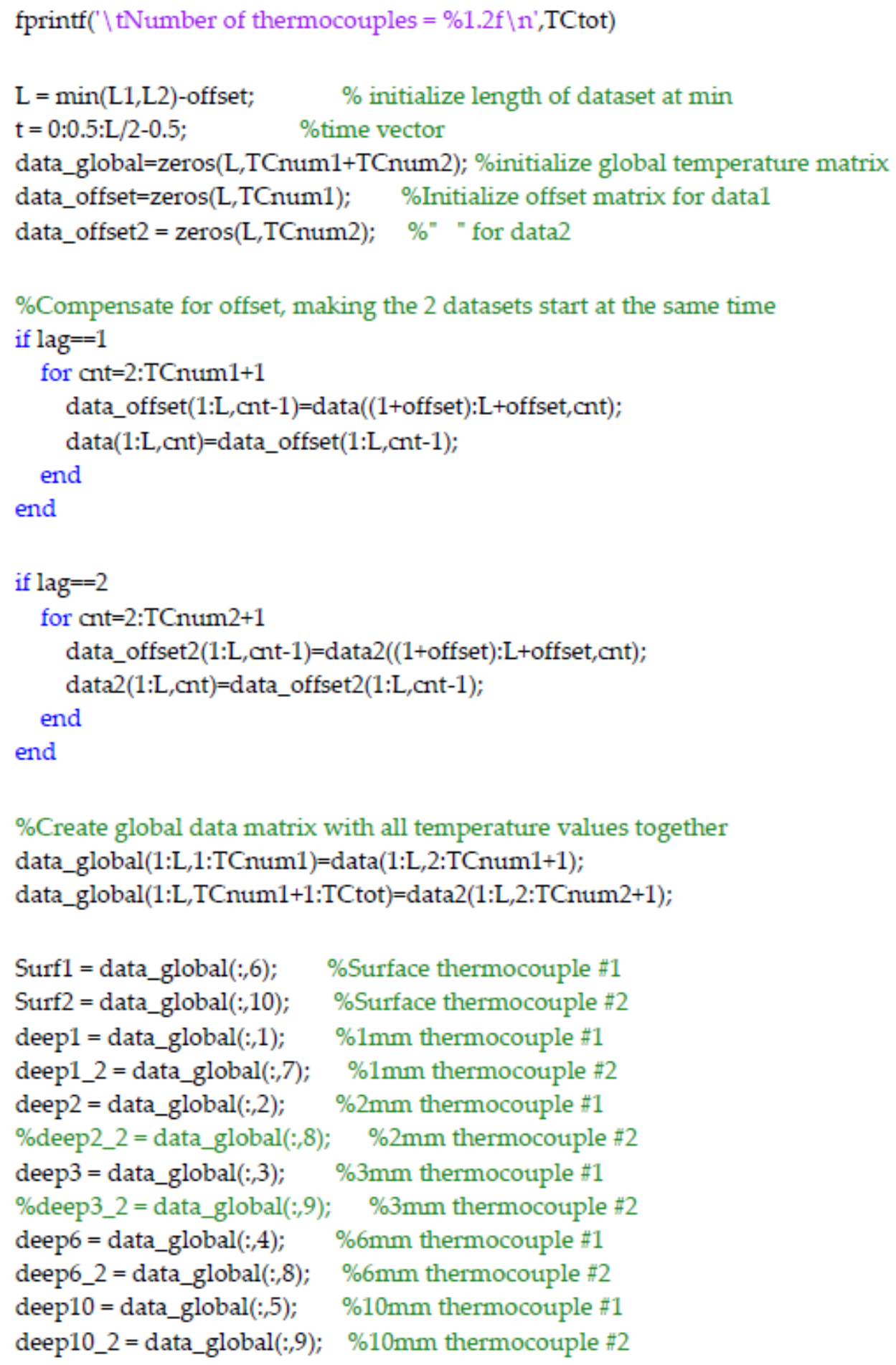




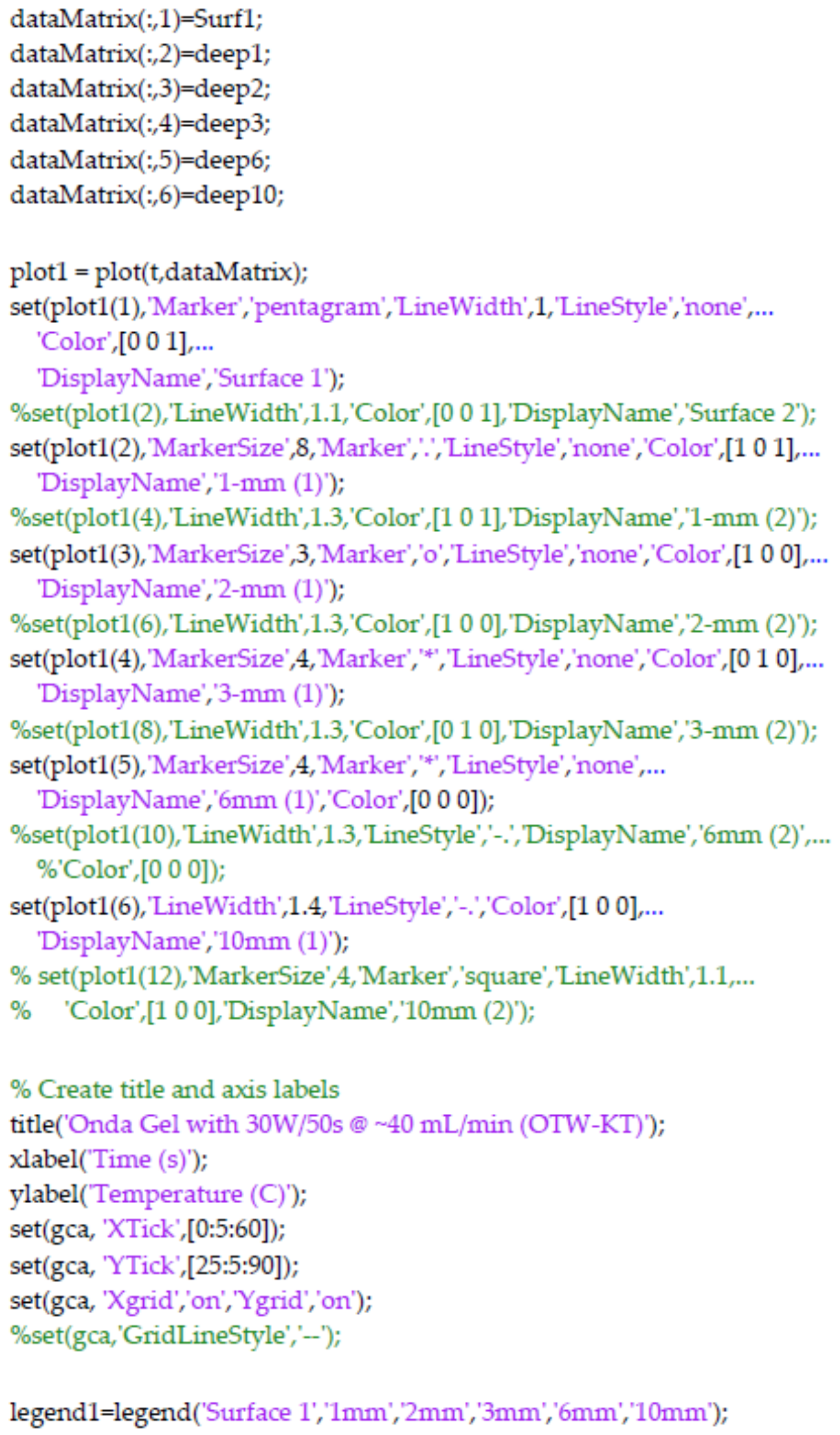

